\title{
DESIGN AND DEVELOPMENT OF A STAIR ASCENSION ASSISTIVE DEVICE FOR TRANSFEMORAL AMPUTEES
}

\author{
A Thesis Presented to the Faculty of \\ California Polytechnic State University, San Luis Obispo
}

In Partial Fulfillment of the Requirements for the Degree Master of Science in Biomedical Engineering

By

Casey Michael Barbarino

June, 2013 
(C) 2013

Casey Michael Barbarino

ALL RIGHTS RESERVED 
TITLE:

AUTHOR:

DATE SUBMITTED:

COMMITTEE CHAIR:

COMMITTEE MEMBER:

COMMITTEE MEMBER:
Design and Development of a Stair Ascension Assistive Device for Transfemoral Amputees

Casey Michael Barbarino

June 2013

Lily Laiho, Ph.D., Associate Professor Biomedical \& General Engineering Department California Polytechnic State University, San Luis Obispo

Richard Savage, Ph.D., Professor Biomedical \& General Engineering Department California Polytechnic State University, San Luis Obispo

David Clague, Ph.D., Associate Professor Biomedical \& General Engineering Department California Polytechnic State University, San Luis Obispo 


\author{
ABSTRACT \\ Design and Development of a Stair Ascension Assistive \\ Device for Transfemoral Amputees \\ Casey Michael Barbarino
}

Transfemoral amputees around the world experience increased difficulty in climbing stairs due to lack of muscle, balance, and other factors. The loss of a lower limb greatly diminishes the amount of natural force generation provided that is necessary to propel oneself up stairs. This study investigated possible solutions to the problem of stair ascension for transfemoral amputees by the means of designing and developing an externally attachable device to a prosthesis. The number of amputations from military service has greatly increased since 2008 , which shows there is a clear need for assistive devices (Wenke, Krueger, \& Ficke, 2012). With the number of amputations rising and no current externally attachable products on the market to aid in stair ascension for transfemoral amputees, the need for this specific device has become more prominent.

Research, previous work, and preliminary testing provided a basis for design and development of a new prototype. Bench top testing was conducted to review concepts in the prototype and provide data for further modifications. Results from testing of previous work, as well as testing of new concepts and modifications, provided a framework for designing a new externally attachable device for assistance in stair ascension. A new prototype was then designed, manufactured, and tested with bench models as well as realtime testing with amputees. Success of the device's performance was based on bench top results and feedback from amputees, noting both the advantages and shortcomings of the 
new prototype. Testing provided results and feedback that the device was well built and functioned properly, but did not perform satisfactorily, particularly in the categories of force generation and balance. 


\section{ACKNOWLEDGEMENTS}

I would first like to thank QL+ for sponsoring this project and including me as part of the QL+ student team. Jon and Scott Monett, I want to thank you both personally for being so supportive and helpful throughout the past few years. Your devotion to QL+ does not go unnoticed, and I am proud to have been able to work with you both. I appreciate your support and confidence in me.

Mom (Linda Barbarino), Dad (Russell Barbarino), and Kevin (my older brother), thank you for providing me with the love, support, and confidence that you have throughout my life and college career.

Dr. Lily Laiho, I would also like to thank you for taking me on as a thesis candidate. Your advice, support, kindness and encouragement have been both helpful and uplifting throughout this process.

I would also like to thank Matt Titchenal, Kimberley Glaeser, and Ryan Mensing for being such a great team during previous work as a senior project team, as well as Dr. Tom Mase for advising our senior project. You all were a pleasure to work with and contributed greatly to the foundations of this thesis. To all students that participated in the QL+ Team Tech competition, thank you for your hard work and dedication during the initial phase of this challenge.

Lastly, a big thank you to Steve Springer, all the staff and patients that participated in testing at WRNMMC, Cameron Clapp, and Owen Beck for being so willing to help in any way possible. This project could not have been completed without your help and feedback with testing. 


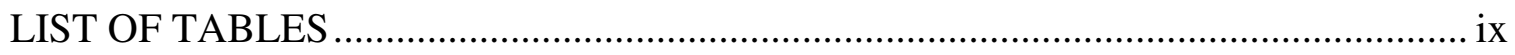

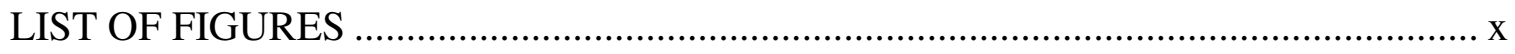

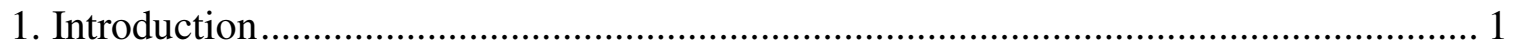

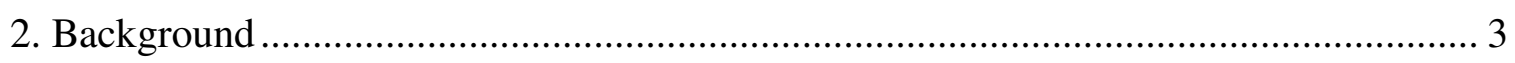

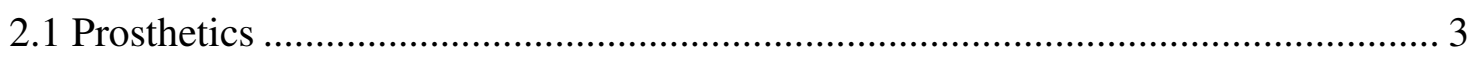

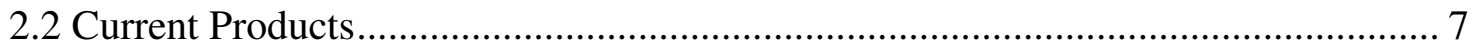

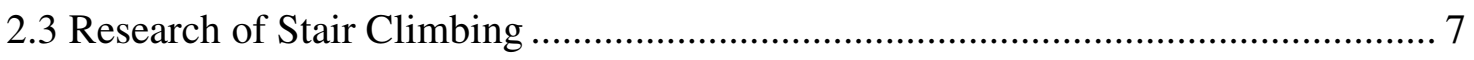

2.4 Previous Work ............................................................................................. 11

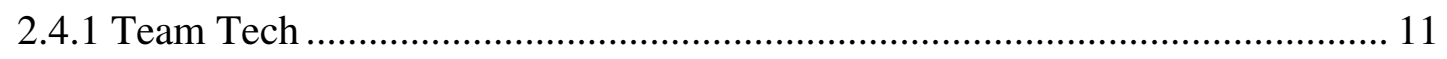

2.4.2 Senior Project ……………………………….................................... 13

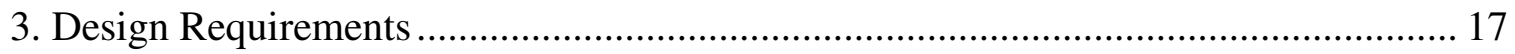

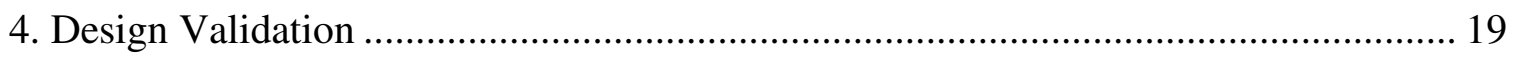

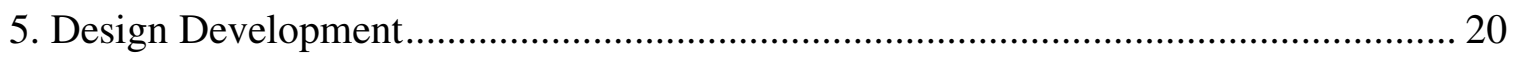

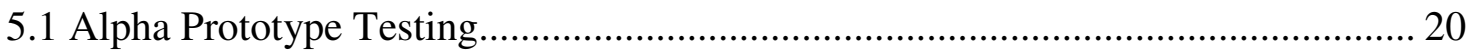

5.2 Prototype Revision Concepts ........................................................................... 27

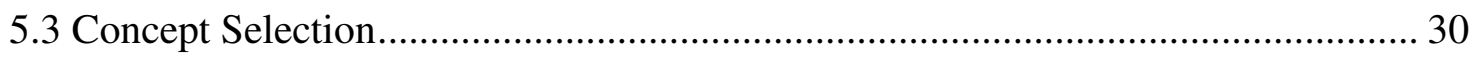

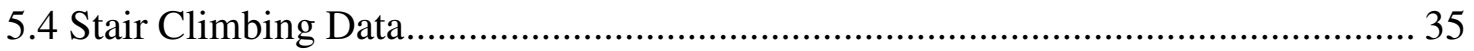

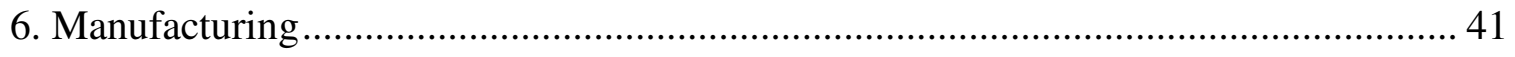

6.1 Custom Shaft ............................................................................................. 41

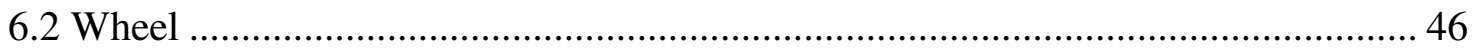

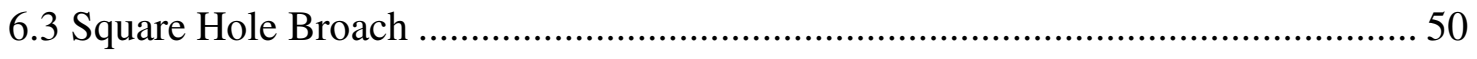

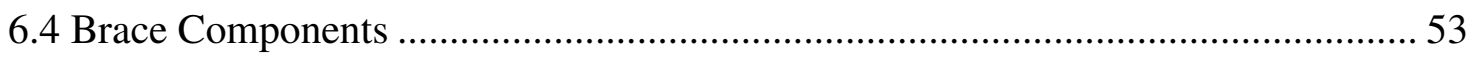

6.5 Adjustable and Velcro Straps .......................................................................... 58

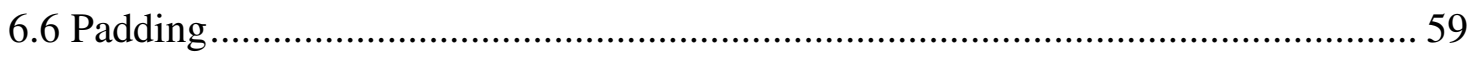

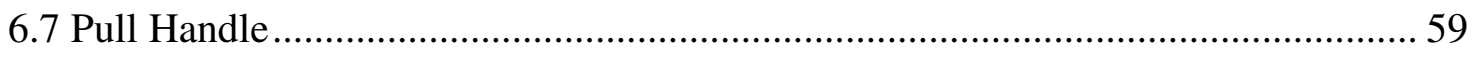

6.8 Bungee Attachment ....................................................................................... 59

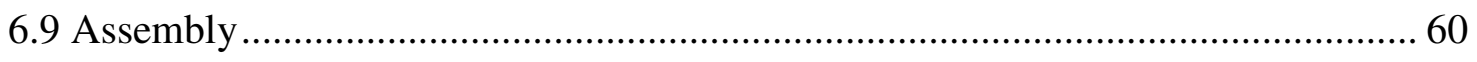




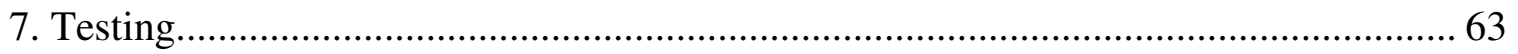

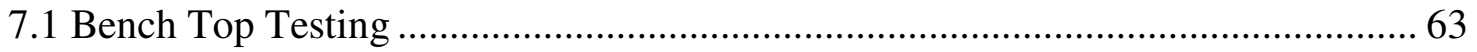

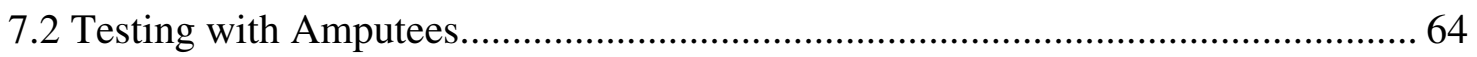

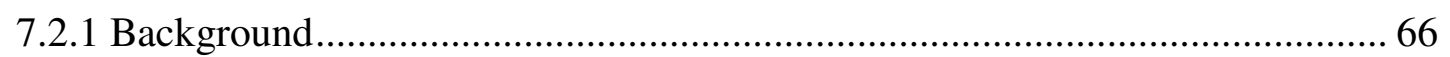

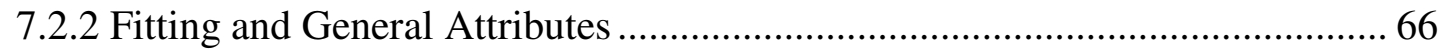

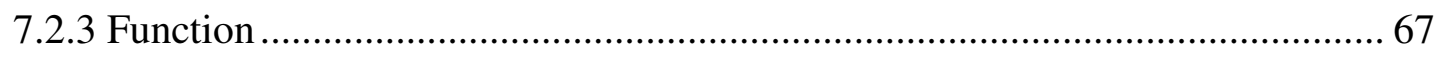

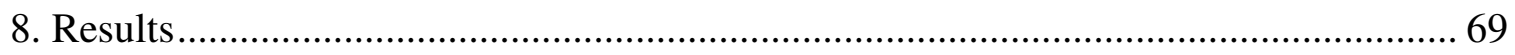

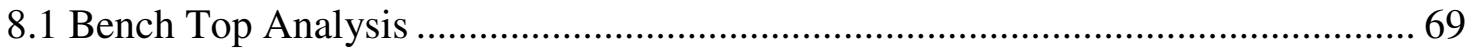

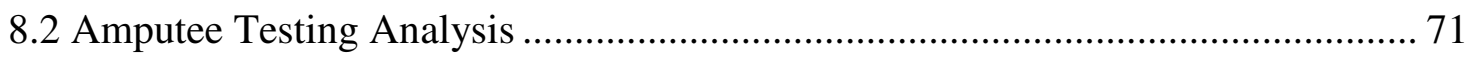

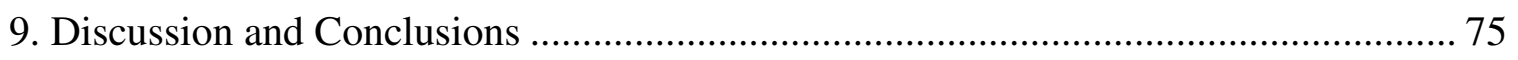

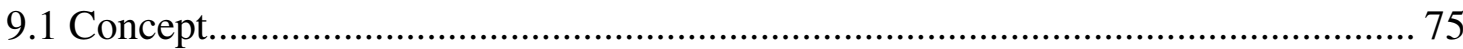

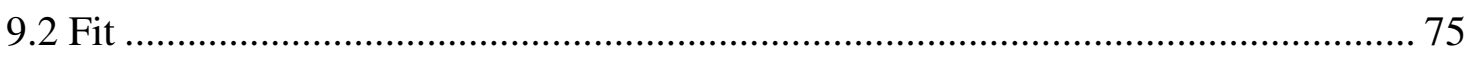

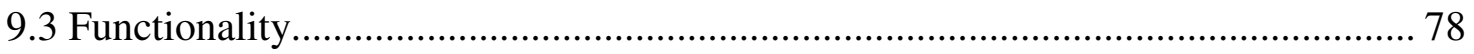

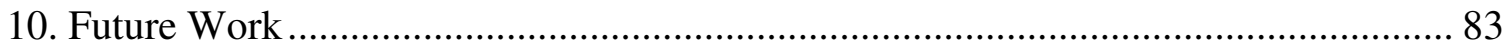

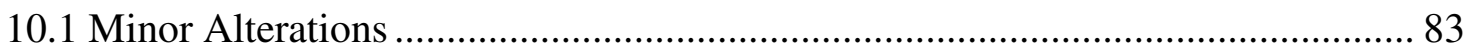

10.2 Current Manufacturing and Design Changes .................................................. 84

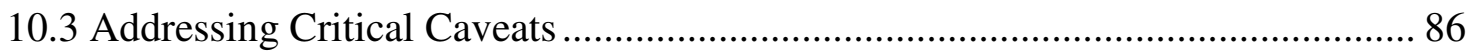

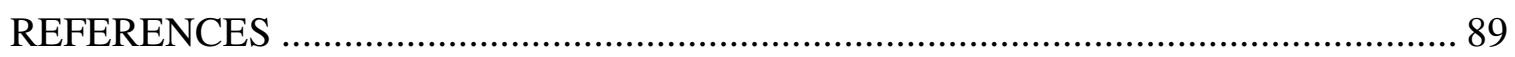

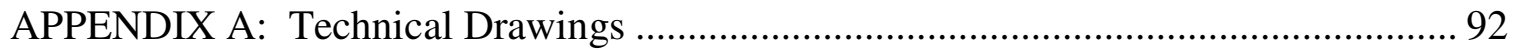

APPENDIX B: Testing Evaluation Forms ……………………………………….... 100 


\section{LIST OF TABLES}

Table 1: Design Requirements for Stair Assist Device............................ 17

Table 2: Maximum Forces and Averages from Force Plate Stair Climbing Model......39

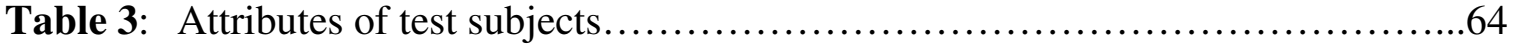

Table 4: Average ratings for qualitative categories of testing with amputees...........72 


\section{LIST OF FIGURES}

Figure 1: Fully assembled new prototype …...................................................... 2

Figure 2: Mechanical and microprocessor equipped prosthetic legs ........................... 4

Figure 3: Anatomy of the legs of a unilateral AK amputee........................................ 8

Figure 4: Peak moments at three lower-extremity joints during stair climbing stud.... 10

Figure 5: Initial prototype of automated and electrically powered stair assist device.. 12

Figure 6: Fully assembled and completed alpha prototype. ................................... 14

Figure 7: Simplified bench top testing setup of new design concept ........................ 15

Figure 8: Final bench top testing setup of alpha prototype..................................... 21

Figure 9: Torque generation of three bungee cords with alpha prototype.................. 22

Figure 10: Inner shaft assembly of alpha prototype.............................................. 25

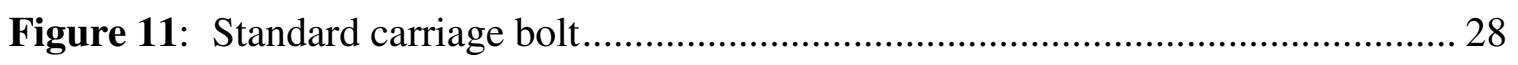

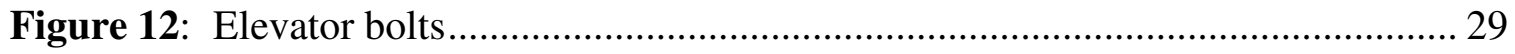

Figure 13: Custom designed and machined shaft with square head ........................... 30

Figure 14: Locking system designs of alpha and adjustable prototype ....................... 33

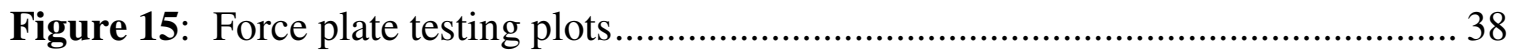

Figure 16: Fully assembled CAD model of new adjustable prototype ....................... 41

Figure 17: Machining setup for key way slot in the custom shaft ............................ 44

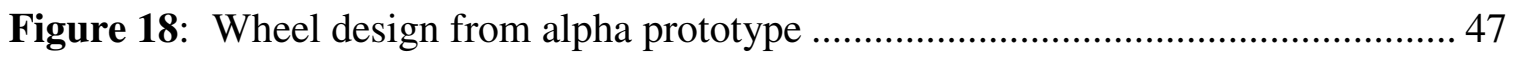

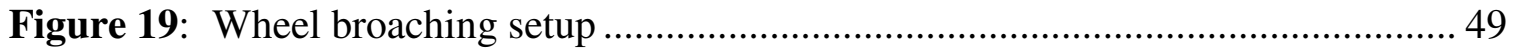

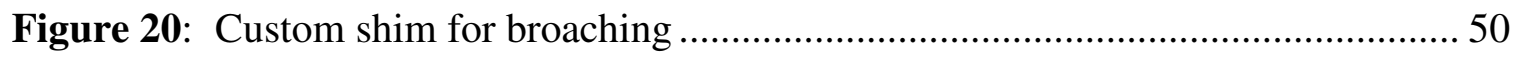

Figure 21: Brace component with the completed square hole ................................. 51

Figure 22: Broach setup for square hole in brace component ..................................5 52 
Figure 23: The plasma cutter setup for cutting brace components

Figure 24: Setup for drilling of bearing hole in brace component.............................. 55

Figure 25: Brace component with completed bends to contour to prosthesis ............... 56

Figure 26: Before and after of bending brace component ..................................... 57

Figure 27: Testing evaluation form provided for amputees at WRNMMC..............65

Figure 28: Torque generation of three bungee cords with adjustable prototype ........... 70

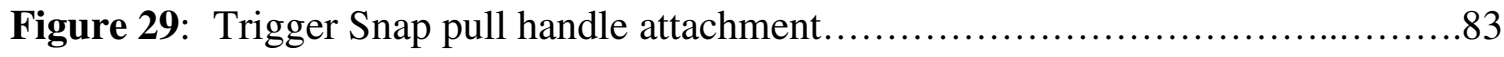

Figure 30: New design for bungee insertion and attachment to wheel....................... 866 


\section{Introduction}

Every year, many men and women return from active military duty with an amputation. In 2011, 240 deployed troops returned with at least an arm or leg amputated (Tan, 2012). Many of these amputees now face an even more tragic event of an above the knee amputation, also known as AK amputations. In addition to adjusting to civilian life, these soldiers must now adjust to a significant daily handicap. Even the most basic of tasks, such as climbing a set of stairs, becomes an extremely challenging feat. There is no escaping stairs and slopes for these users, as these obstacles are ubiquitous in today's world. A recent study by Endolite indicates that the typical lower limb amputee encounters stairs an average of 23 times per day (Zahedi). Since this difficulty is notably common, there is a need for a device that will assist in the climbing of stairs for AK amputees, especially bilateral AK amputees.

Losing a lower limb poses extreme adjustments in muscle availability, stability, balance, and confidence. With the majority of the leg missing, there is a lack of muscle and leverage to perform daily tasks such as squatting, stepping, jumping, and especially propelling oneself up a flight of stairs. Force generation greatly decreases with respect to location and extremity of the amputation, therefore necessitating some sort of compensation. Muscle also plays a significant role in stability and balance of the body. Loss of this muscle can result in a loss of stability and balance. With both the lack of force generation and decrease in stability and balance, it is understandable that many patients would lose some of their confidence in completing certain daily tasks such as those described above. It is these basic principles, as well as a direct challenge posed by Quality of Life $+(\mathrm{QL}+)$, that provided the motivation for this thesis project. More 
specifically, it is the goal of this thesis project to produce an original device to assist amputees in the climbing of stairs and inclines, as shown in Figure 1.

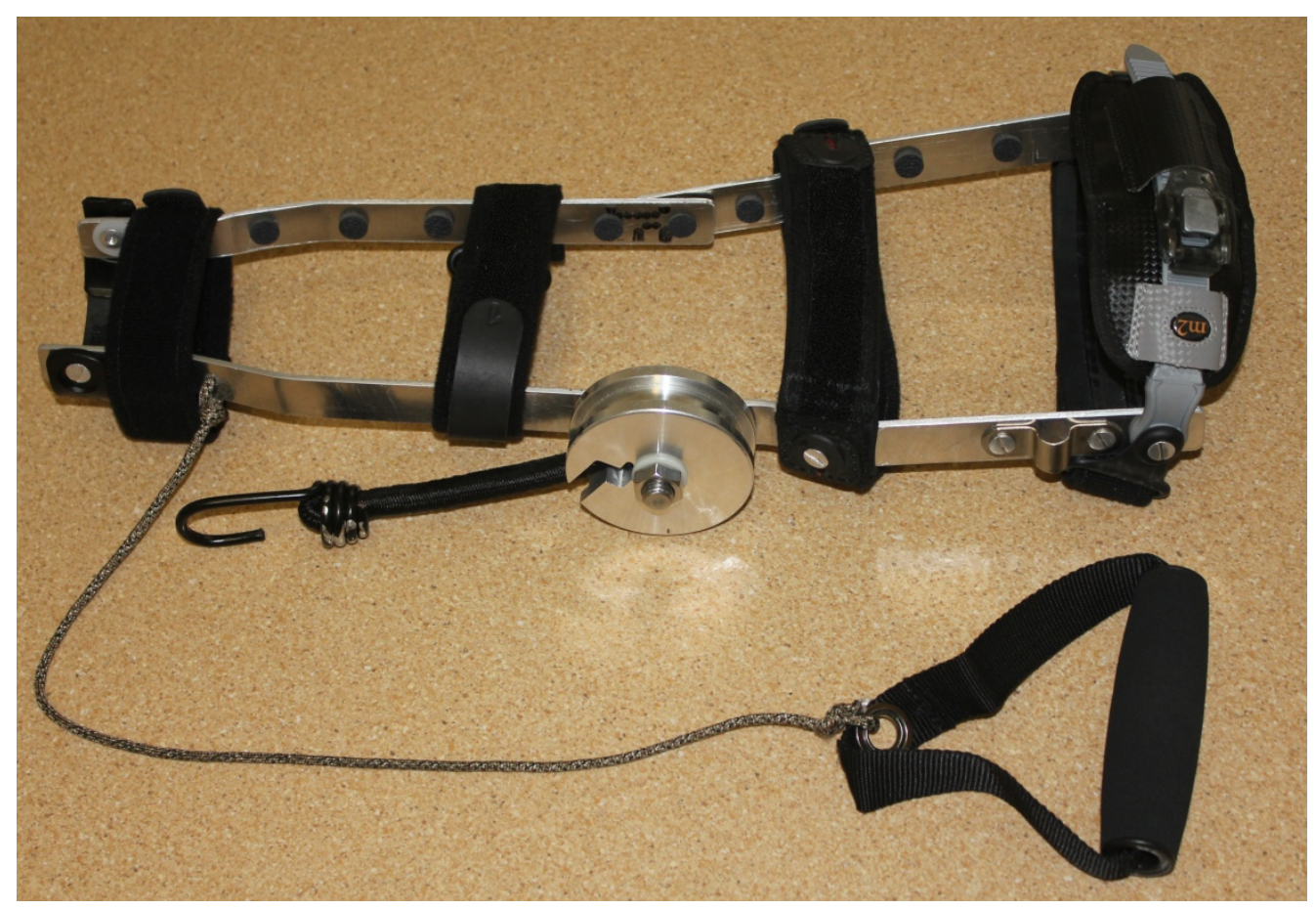

Figure 1: The new, adjustable prototype with all components assembled and ready for use. 


\section{Background}

\subsection{Prosthetics}

Prosthetic devices have been around for ages, with some archaeological findings dating back to $2500 \mathrm{BC}$ in Egypt (History of prosthetics \& orthotics, 2008). Over time, advancements have been made in technology that allow for better design and functionality of prosthetics. A general progression might be starting from a rigid beam to take the place of a leg and knee, to a hydraulic system pivoting about the knee, ending in today's technological wonders of prosthetic legs with microprocessors and motors to introduce the notion of artificial intelligence and better mimic a natural leg.

Prostheses made available to patients in today's world typically range from hydraulic legs to the more intelligent designs including microprocessors and all sorts of motion and force sensors. Even with recent technological advancements, few high end prostheses are designed to aid in force generation, specifically for climbing slopes and stairs. Of all the high end prostheses on the market, the most recognized are typically the C-Leg by Ottobock, the Rheo Knee by Össur, and the Power Knee by Össur, which can be seen in Figure 1. The four different examples of current prosthetic knees in Figure 1 show three microprocessor controlled knees and one purely mechanical knee. Of the three microprocessor controlled knees, the Power Knee, seen on the far right of Figure 2, is the only option that offers an active assist with force generation. An active assist in force generation refers to the creation of force from an object that is not natural to the amputee's body to assist motion rather than using momentum or energy directly from the amputee. 

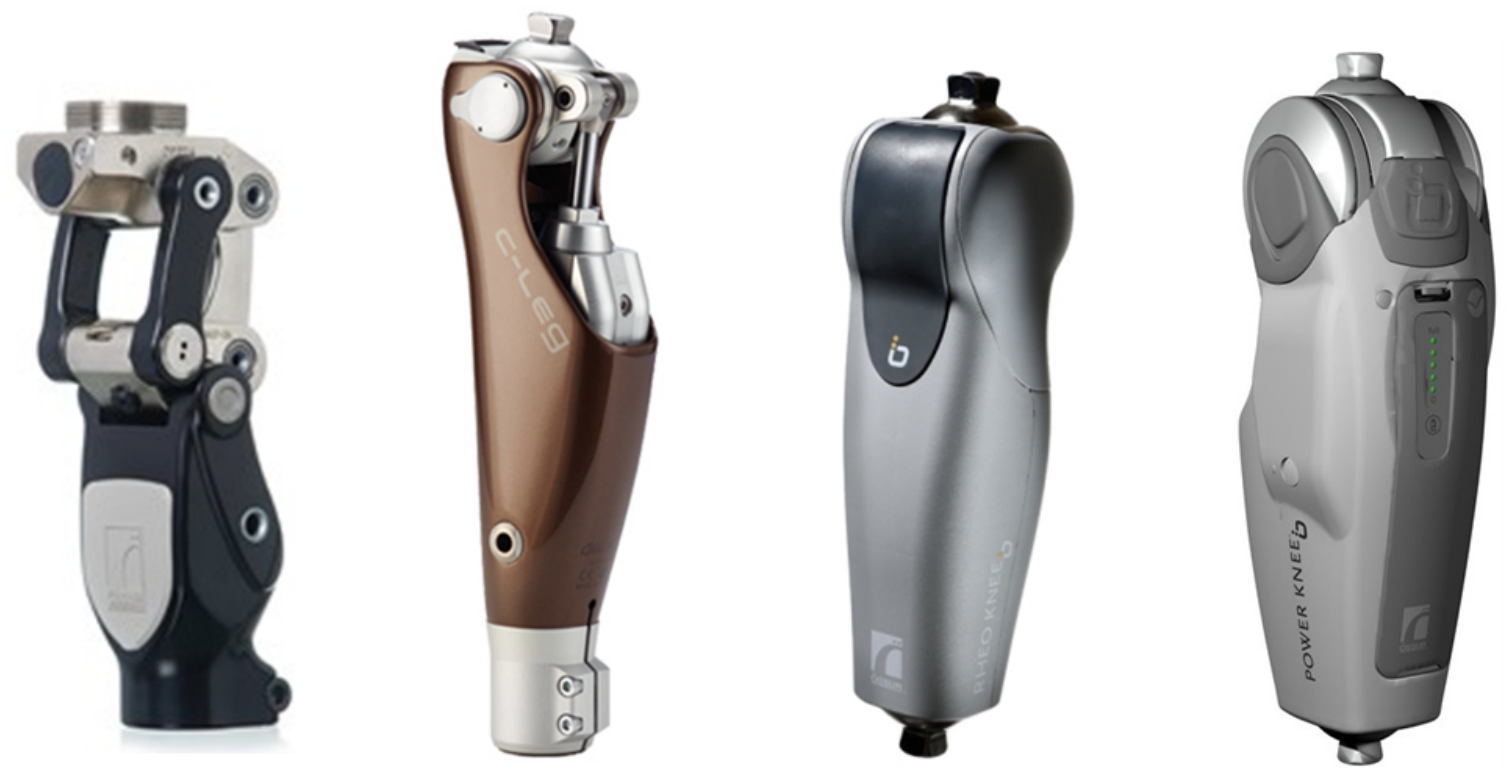

Figure 2: Mechanical and microprocessor equipped prosthetic legs. From left to right: Össur Total Knee (mechanical), Ottobock C-Leg (microprocessor), Össur Rheo Knee (microprocessor), Össur Power Knee (microprocessor with active force generation) (Össur, Total Knee 2000) (Ottobock, C-Leg microprocessor prosthetic knee) (Robots) (Popusoi, 2009).

Devices such as the C-Leg, Rheo Knee, and Power Knee offer tremendous advances in the field of prosthetics. These prostheses are fit with microprocessors, which are what truly set them apart from any standard mechanical knees such as the Total Knee by Össur. These modern devices allow for more personalized settings to control flexion, damping, and many other features. Microprocessors enable the prosthetic to essentially learn the patient's gait and generally daily patterns to better adjust to a natural walking style. Both the C-Leg and the Rheo Knee are designed to improve stumble recovery, where the leg senses a stumble or fall and reacts before the user is aware of the situation (The new functions, 2011) (The technology behind the success of the Rheo Knee). While the Total Knee simply uses a chain of linkages that swivel to function, the C-Leg and 
Rheo Knee use hydraulics systems to provide a natural feeling gait (The new functions, 2011) (The technology behind the success of the Rheo Knee). The C-Leg uses sensors to evaluate stability, and microprocessors will increase resistance if an unstable position is calculated to help recover from trips or stumbles (The new functions, 2011). The Rheo Knee and Power Knee use a similar method for stumble recovery, but also incorporate artificial intelligence to learn about the user over the span of the prosthesis' use (The technology behind the success of the Rheo Knee). The world's first and only prosthetic that is active in aiding with force generation is the Power Knee, made by Össur (The technology of the Power Knee). This device, fit with a microprocessor just like the CLeg and Rheo Knee, also provides many of the same features such as stumble recovery and gait history data recognition (The technology of the Power Knee). However, what sets this prosthesis apart from all others is the electromechanical actuator that can deliver a range of responses such as lifting power to climb stairs and steep grades, slight propulsion for level ground walking, and resistance to aid in descending slopes (The technology of the Power Knee). Although these prosthetics utilize advanced technology including the use of microprocessors and force generation, they are not necessarily suitable for all AK amputees due to factors such as cost, weight, and other factors.

While technologically advanced prosthesis such as the C-Leg, Rheo Knee, and Power Knee drastically increase the quality of everyday life by means of creating a more natural mimicry of the human knee and leg, there are drawbacks that don't allow every amputee to utilize this technology. One major drawback of the more advanced prostheses is their cost to patients and/or insurance companies. The C-Leg can cost between $\$ 40,000$ and $\$ 50,000$, with the Rheo Knee at a similar price range between $\$ 35,000$ and 
$\$ 40,000$ (Austen, 2002) (webpage, 2006). The Power Knee, the only active prosthetic currently on the market to aid in force generation for climbing stairs, can cost as much as $\$ 100,000$ (webpage, 2006). These prices for the prosthetic limbs alone are much more costly for the patient than choosing an alternative basic mechanical leg, which may cost up to $\$ 25,000$ including customization and fitting (webpage, 2006). Not all patients can afford these expensive options for a prosthetic or the insurance to assist in cost absorption. Because of this, many amputees settle for a more basic mechanical knee, such as the Total Knee by Össur. Aside from cost, weight can be another limiting factor in prosthesis selection. Most mechanical or hydraulic knees weigh less than their microprocessor equipped competitors. The Total Knee, a mechanical prosthesis, weighs approximately $1.52 \mathrm{lbs}(690 \mathrm{~g})$, while the C-Leg and Rheo Knee weigh approximately $2.68 \mathrm{lbs}(1215 \mathrm{~g})$ and $3.35 \mathrm{lbs}(1520 \mathrm{~g})$, respectively (Össur, Total Knee 2000) (Ottobock, Otto Bock quality for life) (Össur, Rheo Knee product specificaitons). With more powerful microprocessor controlled prostheses already weighing more than an average mechanical knee, the Power Knee by Össur is not the ideal choice based on weight, weighing in at $6 \mathrm{lbs}(2700 \mathrm{~g})$ without the battery. A heavier prosthesis can be undesirable because it can increase energy expenditure and decrease overall balance of the user. Patients that can afford a microprocessor controlled leg must accept an increase in weight of the prosthesis in order to gain more control, and active assist in the case of the Power Knee. Although there are options for more controlled prostheses that can aid in stair climbing by controlling factors such as balance and force generation, they are typically extremely expensive, as well as heavier than typical mechanical prostheses. With this 
said, there is a lack of a relatively inexpensive, lightweight, and force generating prosthetic made available to amputees around the world.

\subsection{Current Products}

Technology has allowed for intricate microprocessor controlled prosthetic devices to reign over all competitors in functionality of prostheses for $\mathrm{AK}$ amputees. Although there are multiple prostheses to choose from, there is still only one prosthetic device that actively assists in climbing stairs and slopes, the Power Knee by Össur. Currently, however, there are no other devices besides the Power Knee on the market to actively assist $\mathrm{AK}$ amputees in climbing stairs and slopes by means of force generation. There is no option for alternate prostheses or external devices that could attach to a prosthetic leg in order to actively assist with stair climbing.

Based on this research and background, the device being reviewed in this report is a completely original product. Although there are not any external, or prosthesis independent, assistive devices on the market, there are alternative options for force generation to aid in the ascension of stairs and slopes. Thus, the device being reviewed has been designed to be a removable, external assistive device that is made to fit the majority of patients and prostheses by means of adjustability to aid in stair climbing.

\subsection{Research of Stair Climbing}

A basic understanding of the leg is necessary to fully comprehend the monumental loss that an amputee experiences. The human leg is dense with muscles that aid in balance, force generation, and stability. All AK amputees have a unique case, where some are amputated just above the knee joint, while others can be amputated just below the hip. Location of amputation plays a large role in the amount of remaining 
muscle in the leg. Large muscle groups in the leg, such as the quadriceps, hamstrings, gastrocnemius, soleus, and hip flexors, all work together in order to generate enough force to propel oneself up a step, or flight of stairs. With amputation above the knee, entire muscle groups, as well as large portions of remaining muscle groups, are eliminated, leaving a lack of muscle for adequate force generation in situations such as stair climbing as seen in Figure 3. With each leg composing of approximately $1 / 6$ the human body weight, AK amputations create a substantial loss in muscle and mass.

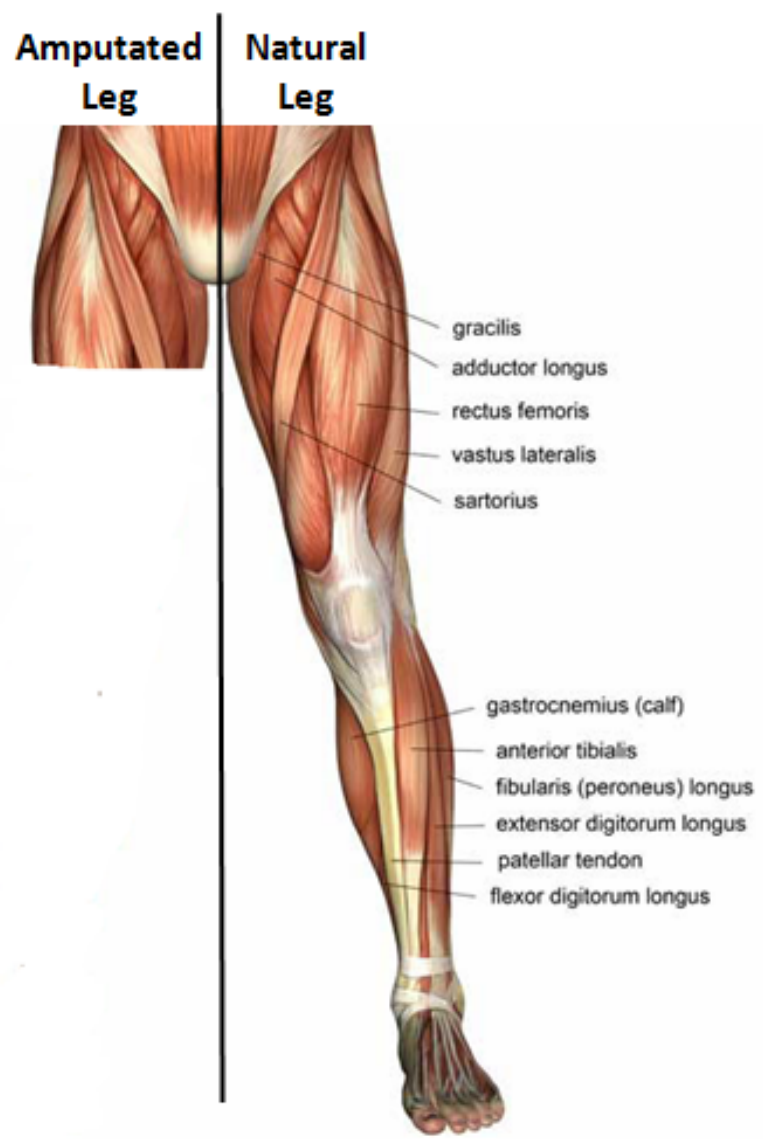

Figure 3: Anatomy of the legs of a unilateral AK amputee. Muscle groupings of a complete leg can be seen on the right, while the left side portrays the remaining stump post-amputation (Trigger point performance therapy: Quads \& IT bands). 
Initial research of the biomechanics of stair ascension was completed to isolate the portions of motion that are most problematic for AK amputees. Both literature reviews and interviews with experts in the field, as well as actual amputees, helped define the physics of the proposed problem. Human ambulation is divided into four distinct phases (Ethier \& Simmons, 2007):

- Stance Phase: leg of focus is in contact with the ground

- Swing Phase: leg of focus is swinging freely (no contact with the ground)

- Heel Strike: the instant the swinging leg's heel contacts the ground

- Toe Off: the instant the swinging leg leaves the ground

Apart from these four phases, there are also five minor motions during any given stage of walking that help to minimize energy expenditure: pelvic tilt, pelvic rotation, knee flexion, ankle flexion, and toe flexion (Ethier \& Simmons, 2007). Together, these motions cause the center of gravity of the individual to oscillate up and down in a characteristic figure " 8 " pattern (Ethier \& Simmons, 2007). Prosthetic users, however, have lost the ability to flex the knee, ankle, and toe at will. In addition, their ability to move the pelvis is limited due to the tightening and/or atrophy of the remaining hip flexor and quadriceps muscles (Ethier \& Simmons, 2007). As a result, their movement is stilted, their center of gravity displaces more vertically, and their associated energy cost of walking is noticeably increased, with a $49 \%$ and $280 \%$ increase in oxygen consumption for unilateral $\mathrm{AK}$ amputees and bilateral $\mathrm{AK}$ amputees, respectively (Huang, 1979).

The most important range of data to inspect, with respect to this problem, is the moment created about the knee upon the climbing of stairs. It is difficult to characterize 
all the different muscles responsible for stair climbing, so the focus is translated to the knee joint, where a moment is created by forces exerted during stair ascent. Using the moment about the knee joint as the main gauge for force generation, or required force, for stair climbing is an approximated simplification for an AK amputee since most of the muscle groups mentioned above are lacking. A torque about the knee joint is required to both bend the knee to plant the foot on a step, and to extend the leg to propel oneself up to the next successive step. With this in mind, approximations of peak moments about the knee from a study on climbing a step onto from either a standing position or walking as seen in Figure 4 will be used to characterize the necessary forces for stair climbing (Vallabhajosula, Yentes, \& Stergiou, 2012).
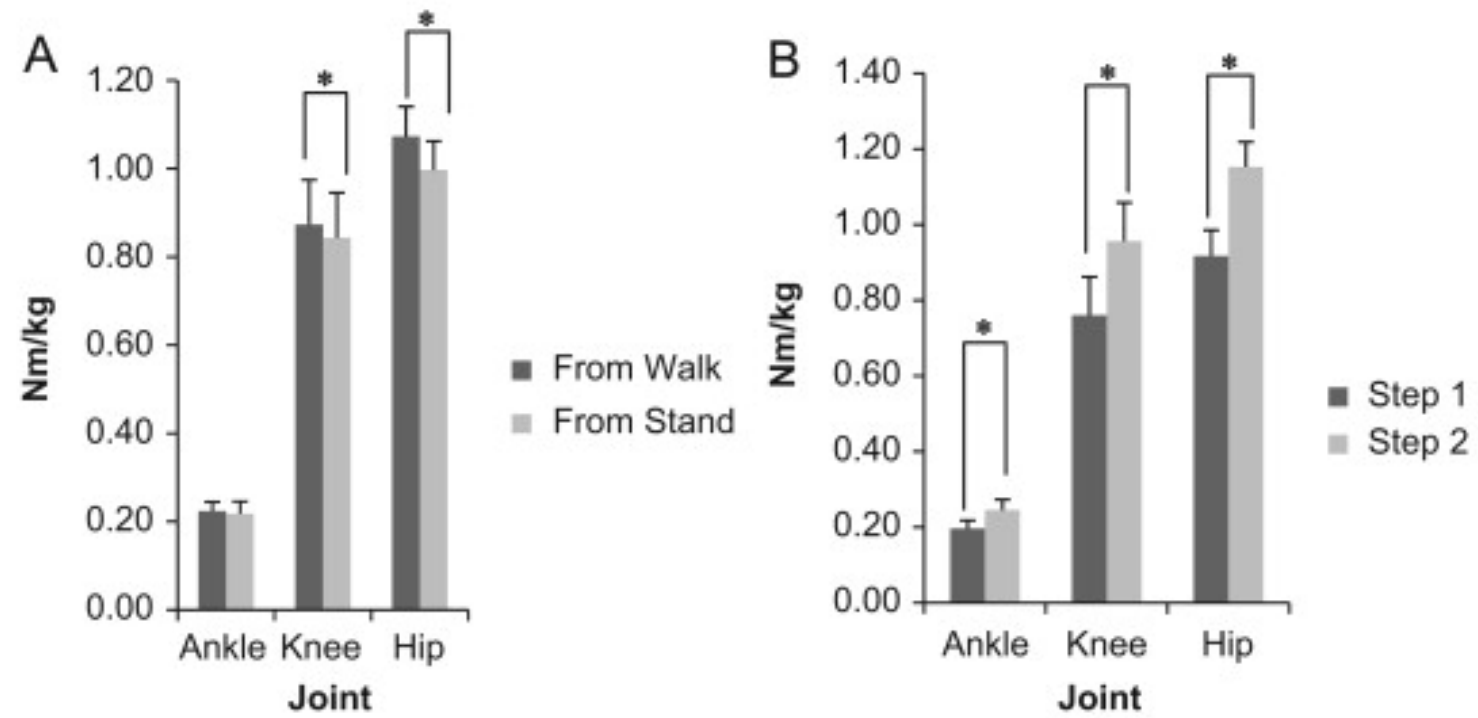

Figure 4: Peak moments and power generation at three lower-extremity joints averaged across the two conditions and across the two steps. Chart A and B are of concern, specifically the knee joint moments, to characterize a range of torque applied about the knee joint during stair ascent (Vallabhajosula, Yentes, \& Stergiou, 2012). 
Bar graphs (A) and (B) of Figure 4 display peak moments for all the conditions of the experiment. Specifically, the peak knee joint moments show a range of approximately $0.775 \mathrm{~N} \cdot \mathrm{m} / \mathrm{kg}$ to $0.95 \mathrm{~N} \cdot \mathrm{m} / \mathrm{kg}$ during stair climbing. Let it be noted that this study was conducted on ten healthy subjects with average weights and heights of $76.2 \pm 13.6 \mathrm{~kg}$ and $1.78 \pm 0.08 \mathrm{~m}$, respectively (Vallabhajosula, Yentes, \& Stergiou, 2012). Patient weight and femur length play a large role in the calculation of the moment force about the knee joint, which can cause variability between patients and studies.

\subsection{Previous Work}

Previous work with this concept has been completed to provide two separate prototypes from differing designs. Work on this concept of an assistive device for climbing stairs was first completed with an on-campus club competing in a design competition. The project was then taken over as a senior project, re-designed, and ended with a prototype.

\subsubsection{Team Tech}

Work on this device first began as a Quality of Life $+(\mathrm{QL}+)$ sponsored club project. A team of approximately 15 students at California Polytechnic State University of California worked together to design and create a solution to the struggle for bilateral AK amputees to climb stairs. After brainstorming, the team selected a design that would allow for complete automation by means of an electronic system. The system incorporated an electronic rotational motor, a machined gearbox to reduce the motor's gear ratio, force and angular sensors, an Arduino Uno microcontroller, an electronics casing, an aluminum brace, and other basic components. The design was hoped to be fit to both legs of an AK amputee to propel them up stairs by means of coordination between 
the angle of knee flexion, force upon the sole of the foot, and power by a motor between the two braces, which can be seen in Figure 5.

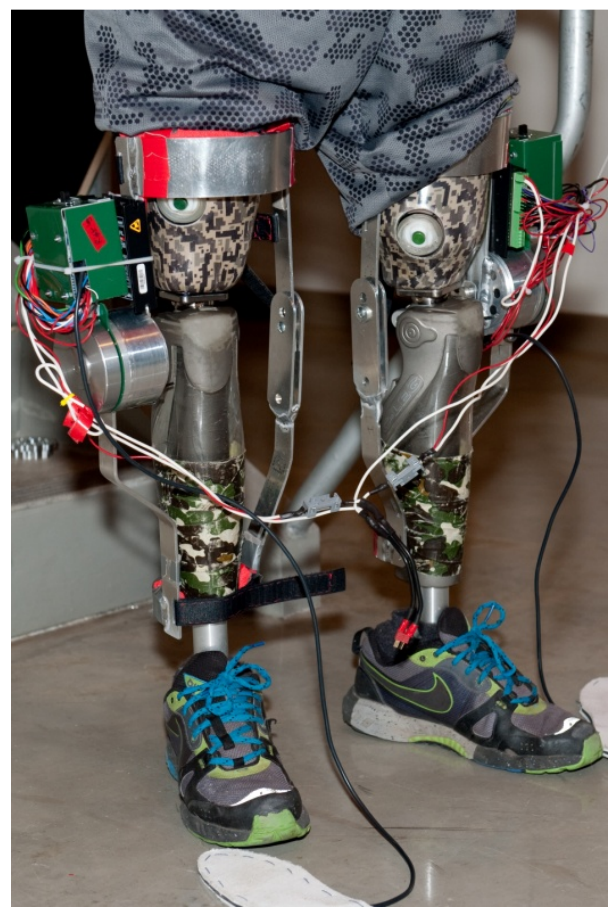

Figure 5: Initial prototype of fully automated and electrically powered stair assist device.

This was designed to be a hands-free, completely automated system to allow the user freedom of their arms for gripping a railing or balance in general. Although a prototype was manufactured, it was not functional due to unfinished programming, a large number of bugs in the written code, sloppy machine work on a gear box resulting in grinding and binding, along with other factors. However, the fit of the prototype was tested on a bilateral AK amputee. Results of fitting, according to the amputee testing the system, was that it was too heavy, obtrusive, loud, and most importantly unpredictable. This automated system was an overall failure in terms of functionality, but it resulted in valuable information for redesign in the future. 


\subsubsection{Senior Project}

Based on the automated, electronic system and its failures in many areas, the design was continued as a senior project including four students of different engineering disciplines. Due to the results and worries posed by the bilateral AK amputee that tested the electronically automated device, a complete redesign was considered in order to introduce more user control, safety, and effectiveness into the device. Initial ideas for redesign included concepts involving bungees, pistons, motors, springs, etc. However, in order to make the device as predictable, controllable, safe, and simple as possible, a purely mechanical design utilizing energy storage in a bungee cord was selected. Based on previous experiences with the project, as well as the previous testing amputee's opinions, the mechanical bungee design best suited the product requirements and specifications agreed upon.

The general design of the device included an external brace made from aluminum, with a custom aluminum wheel that a bungee cord would wrap around during flexion of the leg about the knee. This motion would stretch the bungee cord, therefore storing energy that would be released at the user's discretion by means of a pull handle, as seen in Figure 6. 


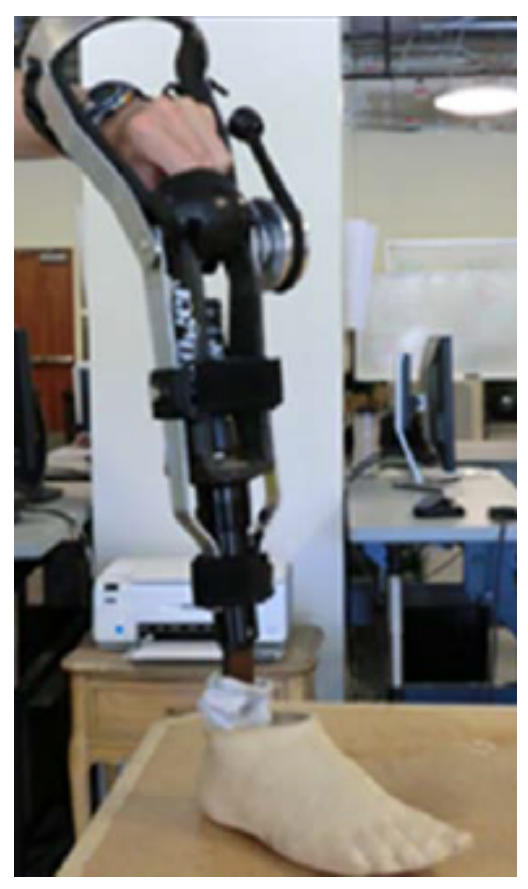

Figure 6: Fully assembled and completed alpha prototype (Barbarino, Glaeser, Mensing, \& Titchenal, 2012).

Initial prototypes of the wheel were made in order to conduct bench top testing of the bungee model. A simplified model of this design was tested to assess the feasibility and force generation. Bench top testing of this new mechanical system, which will be referred to as the alpha prototype from here on out, yielded maximum torque values of approximately $5.75 \mathrm{~N} \cdot \mathrm{m}$ and $5.6 \mathrm{~N} \cdot \mathrm{m}$ applied about the point of rotation, or effectively the knee joint (Barbarino, Glaeser, Mensing, \& Titchenal, 2012). Bench top testing was conducted with a rapid prototyped wheel, continuous lengths of $1 / 2$ " diameter bungee gripped at two different lengths, and an aluminum bench model of the brace, as shown in Figure 7. 


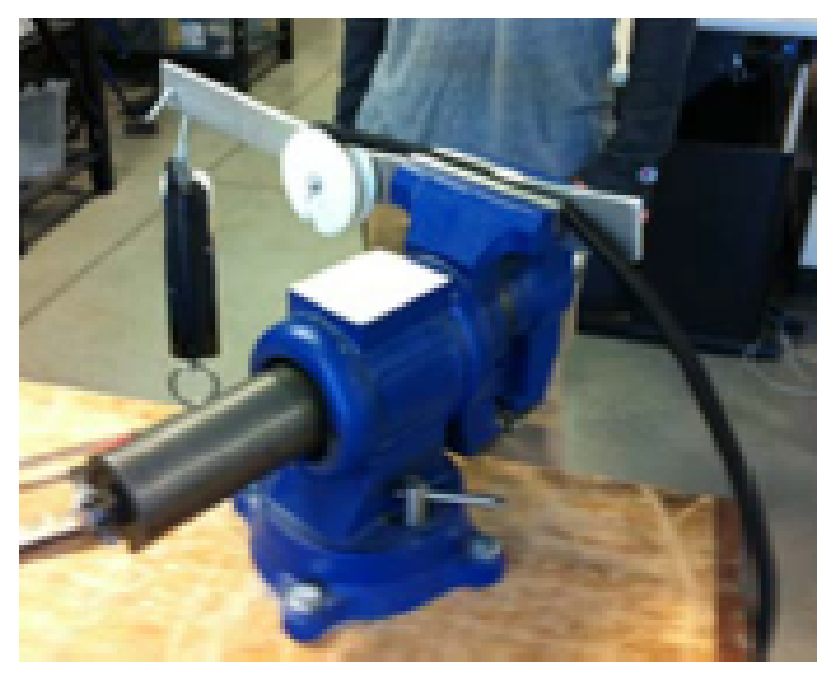

Figure 7: Simplified bench top testing setup for feasibility and force generation of new design concept (Barbarino, Glaeser, Mensing, \& Titchenal, 2012).

Testing and results were based on two different "pre-stretched" conditions of the bungee being held in the wheel and vise. It was later noted, however, that testing in this manner was inaccurate in comparison to later bench top testing. The bungees used in this preliminary bench top testing were effectively shorter than the bungees used in later testing because of the location of grip with the vise, which provided more force than would be possible with the bungee and hook actually used in the final design.

Due to the fact that the shortest possible length of bungee that could be manufactured and purchased for the final design was 6", the bungee is the limiting factor in this simplified bench top testing. This resulted in greater torques applied about the theoretical knee joint than would be expected with the final bungee product with one end free, and the other with a fixed hook. An aluminum brace was custom designed to fit a specific test subject's hydraulic knee and socket. The alpha prototype was manufactured and assembled into a working prototype, which is shown in Figure 5. However, testing of the actual prototype was not completed due to lack of time and location conflicts with 
the amputee that had been assisting in the development and sizing of the device. The end point of the senior project was a finished prototype with no testing conducted on either fit or function by an amputee. 


\section{Design Requirements}

Design requirements for the stair assist device were very similar to requirements listed in previous work. Additional requirements for balance and adjustability were added to the overall requirements to better encompass the entire scope of the challenge, as seen in Table I. The selected design requirements were formed from speaking with amputees about difficulties of climbing stairs, shortcomings of current prostheses, and personal preference on aesthetics and sound. Amputees explained preferences, such as keeping weight to a maximum of $5 \mathrm{lbs}$. Requirements such as supported load and cost were calculated based on average forces and weights of amputees, and cost of prostheses and orthotic braces, respectively.

Table I: Design Requirements for Stair Assist Device

\begin{tabular}{|c|c|c|c|c|c|}
\hline Spec. \# & $\begin{array}{c}\text { Parameter } \\
\text { Description }\end{array}$ & $\begin{array}{c}\text { Requirement } \\
\text { or Target }\end{array}$ & Tolerance & Risk & Compliance \\
\hline 1 & Weight & $5 \mathrm{lbs}$ & Max & M & A, T \\
\hline 2 & Noise Level & Low & Max & L & A, T \\
\hline 3 & Supported Load & $250 \mathrm{lbs}$ & Min & M & A, T \\
\hline 4 & Durability & $1-2$ years & Min & M & A, T, I \\
\hline 5 & Secure Fit & & & M & I \\
\hline 6 & Cost & $\$ 250$ & Max & L & A \\
\hline 7 & Ease of Use & & & M & A, I \\
\hline 8 & User Force Input & 25 lbs & Max & M & A, T, I \\
\hline 9 & Unobtrusive & & & H & I \\
\hline 10 & $\begin{array}{c}\text { Minimal Decrease } \\
\text { in Balance }\end{array}$ & & & H & T \\
\hline 11 & Adjustability & & & L & T, I \\
\hline
\end{tabular}

$\begin{array}{ll}\mathrm{A}=\text { analysis } & \mathrm{L}=\text { low } \\ \mathrm{T}=\text { test } & \mathrm{M}=\text { medium } \\ \mathrm{I}=\text { inspection } & \mathrm{H}=\text { High }\end{array}$


Parameters for requirements were selected to both please the amputees that would be using the device, as well as the engineering specifications necessary. All requirements would be evaluated for compliance by analysis, testing, and/or inspection. Weight of the device was an important requirement according to amputees. If the device was heavy in any sense, it would require more energy expenditure from the amputees than wanted, as well as change weight distribution and therefore diminish balance. Noise level of the device was selected as a requirement due to amputee preference. It was noted that an amputee would not be likely to use the device if it was noisy or made them more self conscious about their prostheses in general. Supported load of the device was based on average weight of amputees, with an increased maximum to a point where it would be difficult for the amputee to climb stairs due to lack of sufficient muscle for their weight ratio.

Durability and cost were selected with one another in mind. Cost was based on current prices of prostheses, which can be extremely expensive as described previously. With this in mind, it was decided that a relatively inexpensive device would be beneficial since amputees have already spent large amounts of money due to their condition. Durability would be a function of cost in some ways. For example, material used for the device could be relatively inexpensive, but would not last as long. However, more expensive materials, such as titanium, could have been utilized to last longer, but would drastically increase the cost of the device. The fit of the device would need to be secure in order for the device to work properly, which is why that was a relatively important requirement. 
Many requirements were selected based solely on feedback from amputees. These requirements include ease of use, user force input, unobtrusiveness, minimal decrease in balance, and adjustability. Amputees explained that if these requirements were not adequately accomplished, the device would not be worth using. With these requirements, the design process was started from the end point of the previous work.

\section{Design Validation}

The new design of the device is very similar to the previous alpha prototype. Because no previous testing of the finished alpha prototype was conducted, many design validations for the new design were identical to previous work due to the lack of feedback from the alpha prototype. The brace components are almost identical, the exact wheel from the alpha prototype is being used on the new prototype, padding is similar, attachment of straps was identical, the same bungees were utilized, and bungee attachment was identical.

Due to the similarities between the alpha prototype and the new design, many of the design validations were carried from the previous project, such as moment calculations about the knee joint for design of the wheel, shaft and key strength calculations, sizing and ergonomics of the brace components based on research, bearing design, brace design and strength calculations, material selection, as well as other design considerations with less impact (Barbarino, Glaeser, Mensing, \& Titchenal, 2012). One important design validation for the new set of design requirements was adjustability. Because the brace was required to be adjustable, side bands of the brace could not be rigidly attached to opposing sides. It was considered that the device may lose rigidity, therefore not providing enough support for proper energy storage. However, this concern 
was validated conceptually by the idea that the only necessary side of the brace for proper function is the side with the bungee and wheel. The other side of the brace simply provides a means for attachment to the prosthesis. To counter the loss of rigidity provided due to the adjustability, more straps for securing the device were added to the design. With design requirements and validations completed, development of the new device was started.

\section{Design Development}

Development of the new design began with testing of the alpha prototype to gain data and feedback from the previous design. Concepts for prototype revisions were then brainstormed, evaluated, and selected. Lastly, data was collected from a stair climbing model using a force plate to better assess the forces necessary for stair climbing.

\subsection{Alpha Prototype Testing}

Development of the new prototype could not begin until testing information on the previous prototype was acquired. The previous prototype, which will be referred to as the alpha prototype from here on out, needed to be tested first to evaluate its performance and fit, since this testing was not able to be completed as part of the previous work. Bench top testing of the completed alpha prototype was conducted to compare to the previous bench top testing with a simplified bench model. Testing of the finished alpha prototype was carried out in the same general setup and procedure as the previous bench testing. The completed prototype was secured in a vise with the top portion hanging off the side of the table to swing free. With the bungee cord attached, 
weights ranging from $3 \mathrm{lbs}$ to $16 \mathrm{lbs}$ were hung from the top portion of the brace, causing it to rotate until it reached equilibrium, as seen in Figure 8.

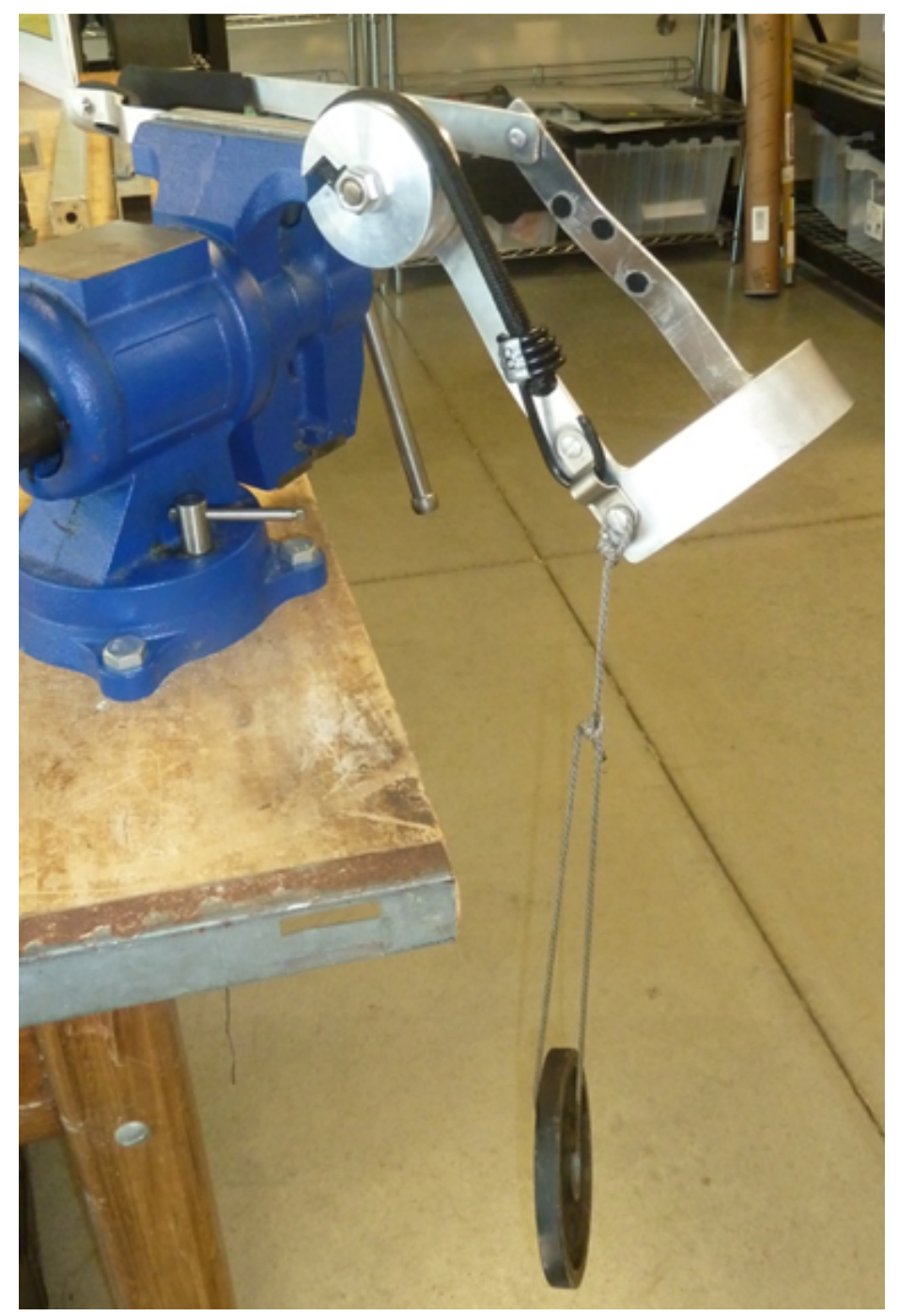

Figure 8: Bench top testing of the alpha prototype was conducted to calculate force generation of each length of bungee.

With each successive weight added, weights and locations of the force application, with respect to angle of flexion, were recorded. This procedure was carried out with a 6", 7", and 8 " bungee with a hook on the end as designed. Moments about the knee joint were 
calculated and plotted to convey the overall force generation of each length of bungee cord, which is shown in Figure 9. After points were plotted, curves were fitted to the data to provide a general shape of the bungee's force generation with respect to degree of flexion, which are represented by solid lines in Figure 9.

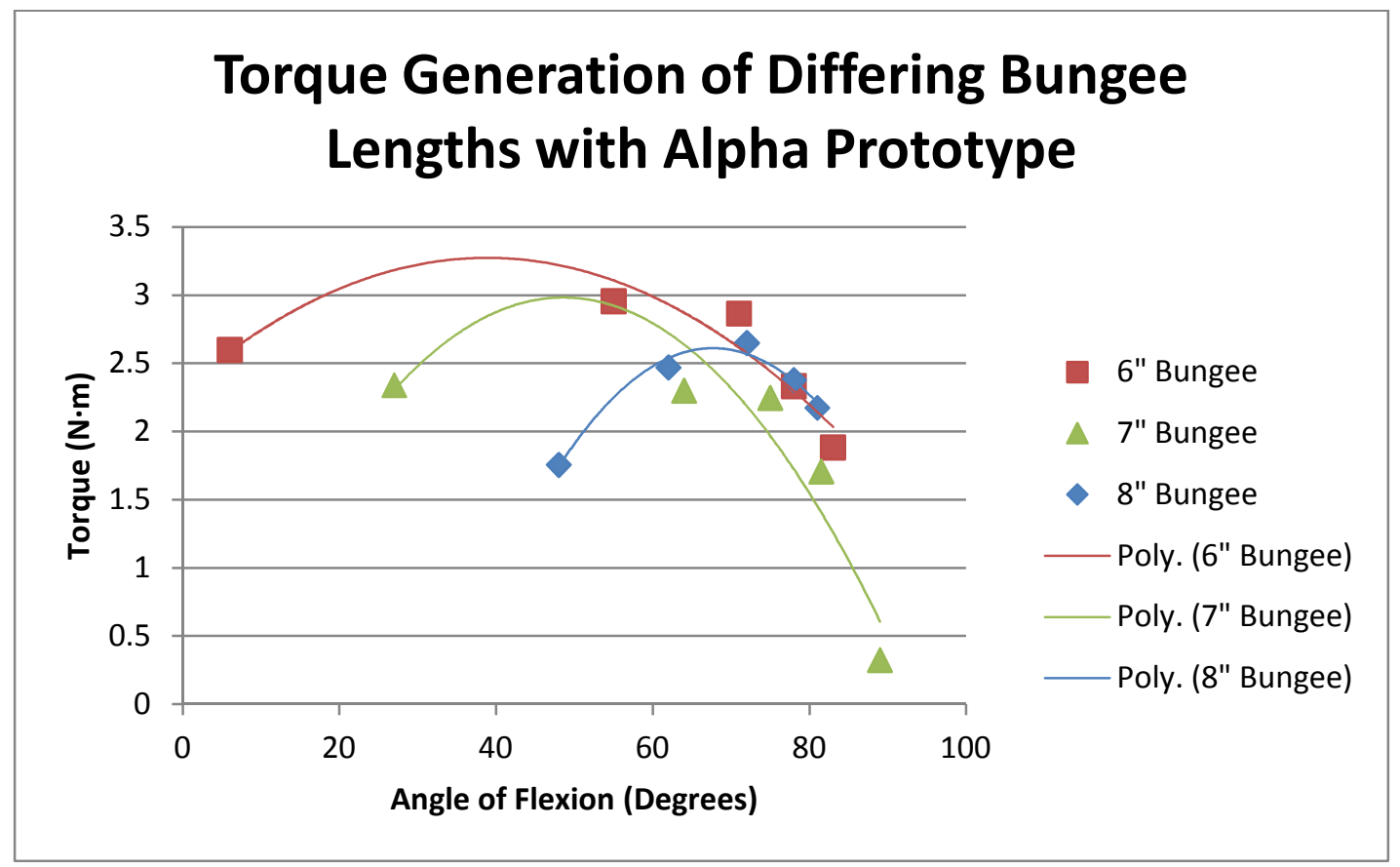

Figure 9: Torque generation of three different length bungee cords from a bench top test with the completed alpha prototype.

Maximum moments of $2.96 \mathrm{~N} \cdot \mathrm{m}, 2.34 \mathrm{~N} \cdot \mathrm{m}$, and $2.65 \mathrm{~N} \cdot \mathrm{m}$ were experienced with the 6", 7", and 8" bungees respectively. When comparing these values to the previous simplified bench top testing, the completed alpha prototype provides approximately half the torque that the simplified bench model generated. This large reduction in force generated can be explained by the setup of the bench models. The differences in the models could be seen in the bungee cord and the wheel. The bungee cord used in the alpha prototype bench top testing included a hook on one end, which was part of the final 
design. This hook allowed for the bungee to be attached to the brace in order to engage the energy storage mechanism once the free end of the bungee was secured in the wheel. The previous bench model, however, did not have a hook on either end. The bungees were secured to the wheel in a similar fashion for both bench models, but the attachment of the other end is what caused the large reduction in force generation. With no hook on the previous bench model, the bungee was attached to the brace by simply clamping it in the vise against the brace. The bungees were all stretched, and then clamped to the brace in order to provide maximum force for the simplified bench model. This effectively shortened the length of the bungee cord drastically, which would create a larger amount of strain, in turn creating more torque. The alpha prototype utilized the bungees with the hook at one end. This design was used to allow the user to easily attach the bungee to the brace to engage the energy storage mechanism for use. However, the supplier of the bungee cords that were used was unable to make a bungee with a hook on one end that was any shorter than 6 " in length due to manufacturing limitations. This limitation is the main reason for the loss in torque. Although a reduction in force was noticed, the exact amount of force required to provide an adequate assist for stair climbing was ambiguous. With this uncertainty in mind, more testing was required to further analyze the efficiency and effectiveness of the device.

To actively test the alpha prototype for fit and function, it was shipped to the orthotics and prosthetics division of Walter Reed National Military Medical Center (WRNMMC) in Maryland, California. The goal was to test the alpha prototype for functionality and fit on multiple AK amputees, both unilateral and bilateral, with different brands and models of prosthetic legs. Upon arrival at WRNMMC, the prototype was 
attempted to be fit on an amputee. Contacts at WRNMMC noted that there were some problematic issues with the device. It was decided that WRNMMC may adjust the brace in any necessary manner to adequately test the prototype. However, before the device could be actively tested, for functionality that is, the shaft pulled out from the brace while adjustments were being made, necessitating re-assembly of multiple components of the prototype. In order to remedy the re-assembly issue, detailed instructions with figures were sent to WRNMMC in order to re-assemble the device. However, contacts at WRNMMC noted that due to their lack of experience and knowledge of the device's design, it would be more time efficient, and most likely more accurate, to ship the brace back for re-assembly and modifications before more testing was conducted.

After more detailed communication with contacts at WRNMMC, a few problems were noted about the device. First, the shaft about which the lower and upper portions of the brace rotate would pull out laterally from its position, which would cause the smaller of the two keys to displace and fall out. This issue would also result in unintentional disassembly of the upper portion of the brace from the lower. In the event that this problem occurs, the device would not function. Second, since the upper and lower portions of the brace were completely rigid due to a two-piece aluminum design pivoting about a joint, there was no adjustability. Because the brace was originally designed for the socket, knee, and pylon of a specific amputee, the dimensions were not suitable for the amputees and their prostheses at WRNMMC. Lastly, it was noted that there was a significant amount of mechanical slop between the shaft, brace, and key system upon rotation. With these issues posed, plans were made to modify the alpha prototype to provide solutions to the noted problems before returning the device for proper testing. 
With the alpha prototype reassembled, a possibility for testing locally in San Luis Obispo, California arose. Unilateral AK amputee, Owen Beck, offered his time and help to test the alpha prototype for qualitative data on fit and functionality during the short remainder of his time in San Luis Obispo. In order to quickly address the problem of the shaft pulling out from the brace, a steel washer was welded to the inner end of the steel shaft to prevent the shaft from pulling out laterally from the brace, which is depicted in Figure 10.

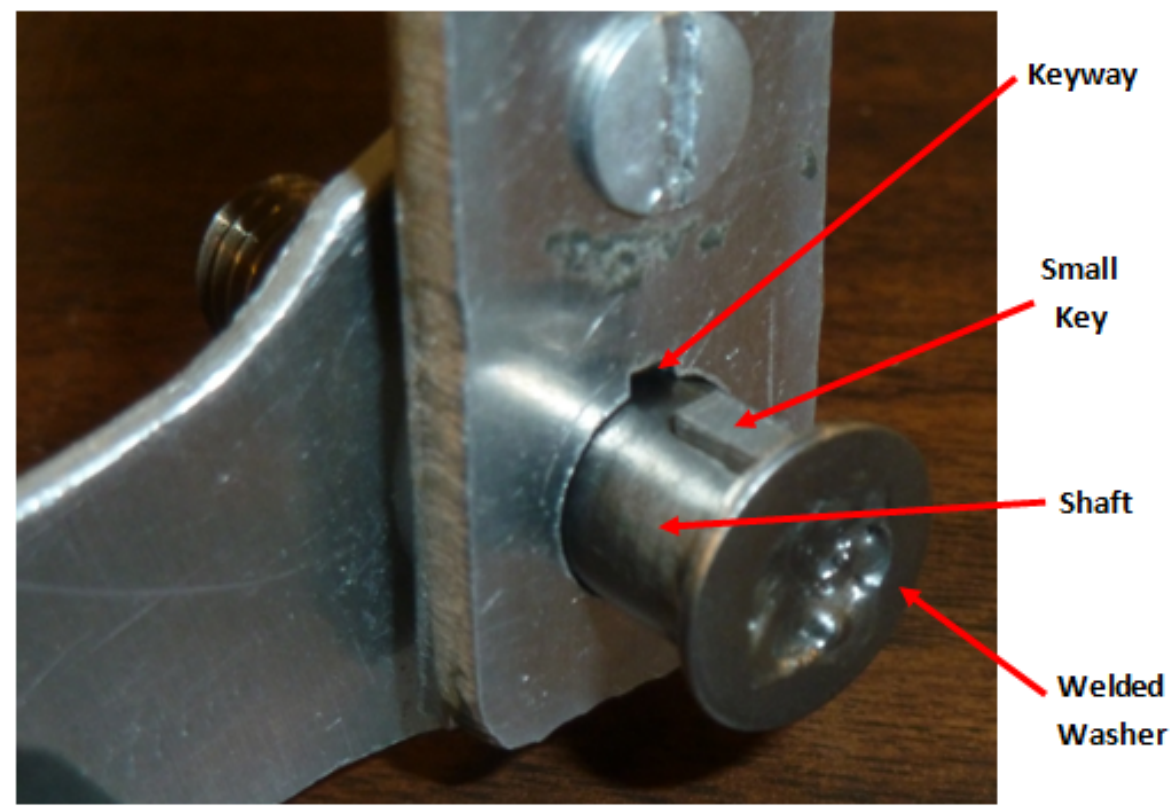

Figure 10: Inner shaft assembly of alpha prototype with a steel washer welded on the end to eliminate risk of the shaft pulling out from the inside of the brace.

The alpha prototype was fit as best as possible to Owen's socket, knee, and lower leg. Due to the custom fit design of the alpha prototype, the brace did not provide an ideal fit for Owen, but the device was secured adequately for testing. Owen communicated and demonstrated how he typically locks his C-Leg in a fully extended and rigid manner while climbing stairs. Owen's technique is unique to his own style and pace of stair 
climbing, where steps are taken two at a time. The locked C-Leg, which is fully extended, is placed on a step, while the natural leg pushes from behind to generate enough force, along with gripping a handrail for balance, to move his center of gravity over and in front of his prosthetic leg, ending with his natural foot two steps above his prosthetic foot. Because the stair assist device cannot function on a locked, rigid leg, Owen decided it was most realistic to put his C-Leg in a free-swing mode for testing, where the knee joint is loose, and the lower leg can swing freely, independent of the upper leg.

Based on testing, Owen provided qualitative analysis of the fit and functionality of the alpha prototype. Although the custom design of the brace did not allow for a proper fit, it was noted that the brace did not seem to secure itself to the brace at enough points, therefore allowing the brace to slip slightly down the leg during use. Along with this issue, it was concluded that the padding on the inside of the brace was too thick, and that adjustability would be beneficial. The minor amount of slip experienced can most likely be attributed to the poor fit of the custom brace to Owen's leg. A general qualitative analysis of the functionality of the alpha prototype resulted in positive feedback. Owen concluded that the forces provided by the device were noticeable during stair ascent. Specifically, it was noted that the device assisted in returning the prosthetic leg to a fully extended position more quickly. The device did not, however, fully propel the test subject up the stair by generating the large amount of force necessary to lift the body up to the subsequent stair, which was expected. With reference to the pull handle for operation, it was noted that the device does not affect balance to an effect that would impede stair climbing. Owen reported that although stair climbing was a slower process 
with the brace, it allowed for more stability and confidence. Unfortunately, Owen only had access to his microprocessor controlled C-Leg prosthetic at the time of testing, resulting in a lack of qualitative data for a mechanical knee to couple with the C-Leg data.

\subsection{Prototype Revision Concepts}

After review from previous testing with both Owen Beck and WRNMMC, it was concluded that revisions to the alpha prototype must be made. These revisions would need to address the issues of the shaft pulling out from the brace, mechanical slop in the system, and general fitting of the brace. Concepts for solutions to these problems were brainstormed, with the intent to not radically change the mode of function of the device.

In order to address the issue of the shaft pulling out of the brace, the concept of welding a washer on the end of the inside of the shaft, which was previously noted and shown. Based on previous testing, the washer solution provided an adequate change to keep the shaft in place. However, the concept of welding a washer onto the shaft did not address the issue of mechanical slop in the system. Because the key system was custom manufactured, there would be no way to alter the original shaft, keys, or wheel to reduce or eliminate slop in the system. Using the washer concept to fix the pull out problem, it was noted that an entire new shaft or wheel would have to be manufactured in order to decrease or rid of the mechanical slop in the key system. The issue of fitting the brace to the prosthetic leg correctly posed another area for change. Since the brace was generally too narrow for both the amputees at WRNMMC and Owen Beck, changes to sizing needed to be made. Solutions to this problem included mechanically bending the current alpha prototype brace to create a wider profile of the upper and lower aluminum bands 
that fit around the socket and pylon, manufacturing a brand new larger brace with wider dimensions, or creating an adjustable design for the whole brace.

After looking at the options available, the washer solution adequately corrected the shaft pull out problem, but was a sloppy and inaccurate solution. Also, this solution only addressed one issue, neglecting the issue of mechanical slop. A more desirable solution would solve both the pull out and slop problems simultaneously. Concepts for simultaneous solutions to the shaft pull out and slop included using a carriage bolt as the shaft, using an elevator bolt as the shaft, or creating a square hole and custom manufactured shaft with a square end and cap to mate with the lower portion of the brace rather than using the previous key method. With a carriage bolt, the square neck would grab all four corners of a square hole, engaging more material than the previous key mechanism, while also providing a larger diameter head to the bolt that would inhibit the bolt from pulling out of the brace, shown in Figure 11.

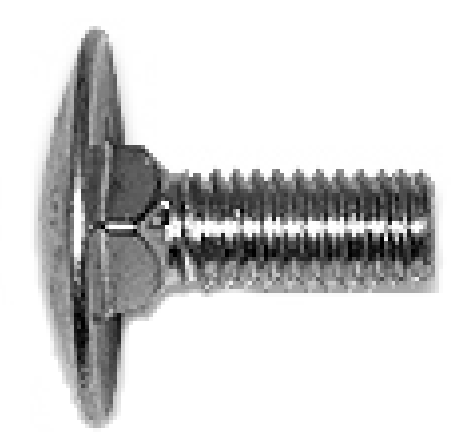

Figure 11: A standard carriage bolt has a button top head, with a square neck, and a threaded shank, which can sometimes have a shoulder. The dimension of the carriage bolt is based on the dimension of the square neck, which is slightly larger than the shank of the bolt (Component parts limited). 
With an elevator bolt, the pull out would be addressed by the large diameter head of the bolt, while the previous key method would have to be utilized again in order to lock the wheel to the lower brace. However, there are also elevator bolts with a square neck, similar to a carriage bolt, in which case the elevator bolt with the square neck would grip the four corners of a square hole just like the carriage bolt, shown in Figure 12.
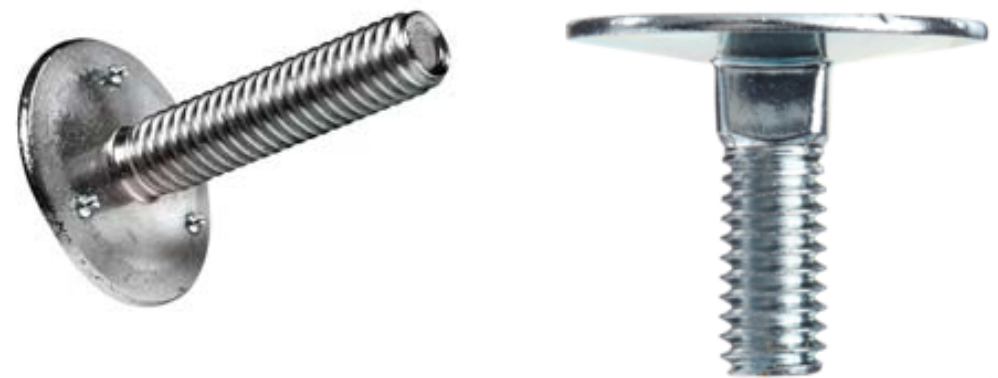

Figure 12: Elevator bolts are manufactured in multiple styles. The left image depicts an elevator bolt with a standard shank and a flat head with small pins on the underside to create a slight grip. The right image depicts an elevator bolt with a square neck, threaded shank that is slightly smaller in dimension than the square neck, and a flat head (Thomasnet) (Amazon supply).

The only difference between the carriage bolt and the elevator bolt with a square neck is the profile of the bolt head. Lastly, a custom shaft with a square end would act like the square neck of the previous bolts discussed, gripping the four corners of a square hole in the lower brace in order to lock the wheel with the lower brace. The custom shaft would address the pull out issue with a tapped hole on the top surface of the square head, where a screw with a large diameter head would be inserted to provide a surface larger than that of the square hole so that the shaft will not pull out, as seen in Figure 13. 


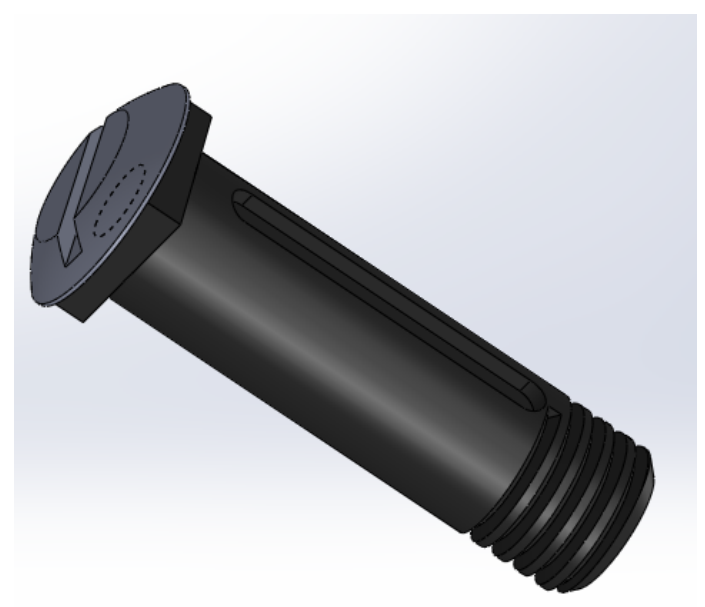

Figure 13: Custom designed and machined shaft with a square head. A wide head, low profile screw would be screwed into a tapped hole to provide a diameter larger than $1 / 2$ " to prevent shaft pull-out.

Using a carriage bolt, elevator bolt, or custom shaft with a square hole would introduce a new locking mechanism between the shaft and the lower portion of the brace that mates over a larger area, creating a more sturdy connection to allow a 1:1 rotation of the wheel and lower brace without slop.

\subsection{Concept Selection}

With multiple options available for solutions to the issues of mechanical slop, shaft pull out, and fit of the device, selections of these concepts were made based on the following justifications. Based on research, options for fixing the shaft pull out issue led to the selection of a custom machined shaft with a square end and some sort of wide diameter cap. The custom shaft concept was selected due to the fact that a $1 \frac{12}{2}$ diameter shaft was necessary in order to fit properly inside the special bearing used in the shaft and brace assembly. 
Elevator bolts were not a viable option because, based on internet research and phone calls, elevator bolts have a maximum production diameter of $3 / 8$ ", which would not fit properly inside the bearing used. In order to stray away from a redesign of the bearing hole in the brace, finding a new thin section ball bearing to fit the smaller diameter shaft, and overall redesign in general, elevator bolts were immediately eliminated as a solution concept due to their smaller diameter.

Carriage bolts were another concept for a solution to the slop and shaft pull out issue. It seemed that a carriage bolt with a relatively long shoulder would provide an adequate solution to addressing both the slop and shaft pull out simultaneously due to their square neck and wide diameter head. Anatomy of a carriage bolt reveals a round head that is of larger diameter than the rest of the bolt, which would stop the bolt from pulling out. Directly beneath the round head is a square neck, which would lock into a square hole in the lower brace, rather than using the previous key method to lock the lower brace to the shaft. Because the key in the previous prototype was so small in the section between the shaft and lower brace, specifically $1 / 8$ " wide $\mathrm{X} 3 / 32$ " tall X $1 / 8$ " long, manufacturing was difficult and required tight tolerances, which were not quite achieved, therefore creating mechanical slop between the shaft, key, and brace. The previous key was in contact with approximately $3 / 64$ " x $1 / 8$ " on both the shaft and brace, which is a relatively small portion of material contact, which can be seen in Figure 9. With the square neck on the carriage bolt, there would be four different points of contact with the brace, and there would be no need for a key since the square portion is part of the bolt itself. While the carriage bolt seemed to be the perfect solution, more research revealed that the $1 / 2$ " dimension, which is necessary to fit correctly in the bearing for 
smooth rotation, is only applied to the square neck, and not the shank of the bolt itself. The shank of the bolt was undersized from $1 \frac{1}{2}$ ", and would therefore not fit in the bearing, making it an unreasonable part to use as a solution.

With both of these concepts proving inadequate solutions to the issues at hand, the concept of the custom shaft with a square head and cap was the remaining concept that was selected. This custom shaft would have a square head, or end portion of the cylindrical shaft, just like the neck of the carriage bolt, in order to lock at four points with the lower portion of the brace by means of a square hole to reduce mechanical slop. This new square locking design allows for more area of the shaft to be grabbing against the aluminum frame, specifically at four different corners, each $1 / 2$ " from one another. Four larger points of contact between the shaft and brace allow for a better fit, and better force transfer without failure compared to the previous custom key design, which only allowed for two points of locking the shaft and brace. Although this new design is conceptually more robust and provides more area to grip the brace, therefore increasing the overall pressure and decreasing stress concentrations, detailed comparisons to between the alpha prototype key method and new prototype square hole method were used to ensure that failure will not occur during basic use. The alpha prototype locking system was designed with a key engagement depth of 0.06 " into the 0.125 " thick brace, with two corners on which the key would engage, while the new adjustable prototype locking system was based on a square head in a square hole where there is 0.160 " of engagement at four different corners, as seen in Figure 14. The new method provides a total engagement surface area of $0.16 \mathrm{in}^{2}$, while the alpha prototype design only provides an engagement surface area of only $0.031 \mathrm{in}^{2}$. With an increase in approximately 5.16 times the amount 
of engaged surface area, the new square head and square hole design proves to be superior to resist failure of the brace.
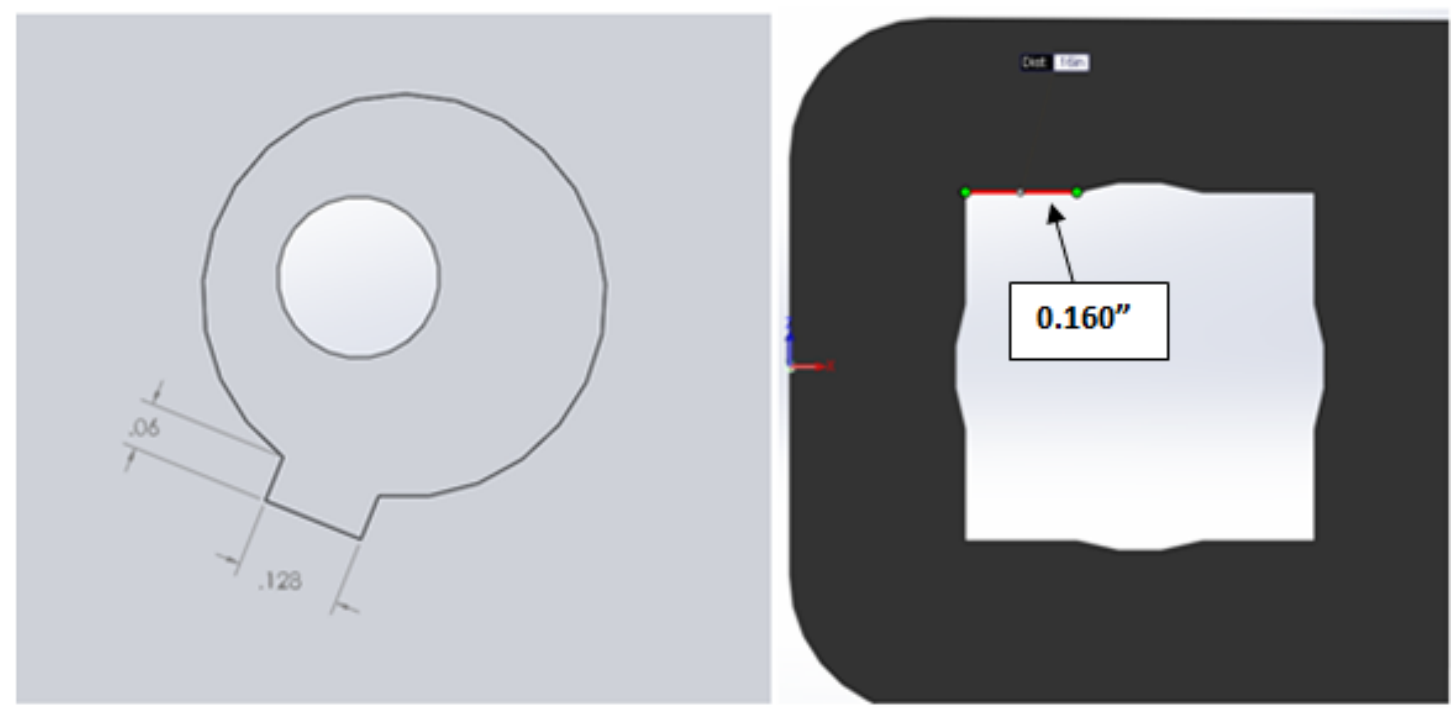

Figure 14: Locking system designs of the alpha prototype and new adjustable prototype show differences in engagement surface area of the locking system based on length of contact and a $1 / 8$ " thick aluminum brace component. The image on the left depicts the alpha prototype locking design, with a small keyway, while the image on the right depicts the new prototype locking design with a square hole in which a square head of the shaft would fit.

The shank of the custom shaft would be $1 / 2$ " in order to fit correctly through the bearing. In order to solve the shaft pull out issue, a hole would be drilled and tapped on the outer face of the square portion. A screw with a head diameter larger than $1 / 2$ " would be screwed into this tapped hole to prevent the shaft from pulling out of the brace from the inside. A shoulder would remain along the shaft from the end of the square head, to a desired length where threads would be machined to secure a nut in order to hold the shaft, wheel, and brace assembly together. 
With regards to the fit of the brace to the overall contour of the prosthetic socket, knee, and lower leg, the concept of a fully adjustable design was selected over modifying the alpha prototype. Altering the alpha prototype to be wider at certain points would be sufficient for allowing a general fit for any prosthetic, but this modification would be undesirable since it would widen the brace dimensions overall, which could allow a larger range of users to fit the brace over their prosthesis, but would restrict any amputees with relatively narrow prostheses in comparison from utilizing the device since the fit would be too loose for proper function. Because of the need to fit all prostheses, the fully adjustable concept was selected. A padded, ratcheting adjustable strap was selected for the highest point of the brace, as well as a simpler adjustable strap on the lowest point of the brace as a replacement to the previous rigid aluminum bands around the socket and pylon. These adjustable straps would allow for total control of the overall width of the brace, which would provide a feasible size for any prosthetic socket, knee, and lower leg. Although the width of the brace would be adjustable, it was determined that the rigid aluminum side bands of the brace should be mechanically bent in order to properly follow the contour of general prostheses to fit the form of the user's prosthesis as best as possible. This new concept would be changing the design of the previous brace from a rigid two component frame where each side was rigidly attached by an upper or lower aluminum band, to a system where each side of the brace is independent of the other due to lack of rigidity in the adjustable straps, therefore allowing adjustments to the width of the brace. Lastly, in order to help further secure the device to the user's prosthesis, multiple adjustable Velcro straps would be utilized to tightly fit the brace to each portion of the prosthesis. The Velcro straps overall length can be adjusted so that they will 
conform to essentially any shape. This fully adjustable design would allow for a larger range of users, as well as provide a more secure fit in general.

Lastly, in order to reduce any extraneous slop in the system after the above modifications, a new keyway system was designed between the shaft and wheel. The previous key system in the alpha prototype was designed to be custom based on Shigley's Mechanical Engineering Design recommendations (Barbarino, Glaeser, Mensing, \& Titchenal, 2012) (Budynas, Nisbett, \& Edward, 2011). The previous custom key, which traveled the length of the entire wheel, was designed and machined to be of rectangular cross section since rectangular keys in the necessary dimensions were not available for purchase. Custom machining created offsets from the desired nominal size due to slop in the mill machines, as well as user error, seeing as it was machined by amateurs. In order to remedy the slop between the shaft key slot, the key itself, and the wheel, a new keyway system was designed with a standard square cross section key. With this new key, no machining was necessary on the key itself, resulting in less slop. However, due to the new key sizing and shape, a new keyway would need to be broached into the wheel, and a new keyway on the shaft would be required.

\subsection{Stair Climbing Data}

Due to the large difference in required torque about the knee necessary to climb stairs from previous calculations and more recent literature review, it was concluded that another set of data would be beneficial to more accurately characterize the feat of stair climbing with respect to force generation. Previous data was calculated based on average weight and height of amputees, as well as average stair step height, which stated that an average of $18 \mathrm{~N} \cdot \mathrm{m}$ about the knee is necessary to climb a stair (Barbarino, Glaeser, 
Mensing, \& Titchenal, 2012). On the other hand, more recent literature review conveyed that a maximum average torque of $72.39 \mathrm{~N} \cdot \mathrm{m}$ is necessary for climbing stairs (Vallabhajosula, Yentes, \& Stergiou, 2012). Because these values are so drastically different, another model was desired to help bridge the gap between the two.

To set up a model for finding torque produced during stair climbing, a force plate was used with a wooden block to simulate a step. After taking measurements of steps on the local campus, it was calculated that the average step was approximately 5.5". To accurately model this, the wooden block used for step simulation was also approximately 5.5 " in height. The force plate was used with a software program called BioWare. This software packaged allowed for force calculation in the $\mathrm{x}, \mathrm{y}$, and z-direction. However, only force in the z-direction was analyzed since the main focus of this model was to approximate vertical forces to ascend a stair step. Multiple step modes were tested to attempt to characterize forces during differing speeds of stair ascent. A slow, medium, and fast step were all tested, with five trials in each mode to produce a relatively substantial data set to minimize error or unwanted variation in average values.

Although this force plate model was not completed with an amputee, precautions were taken to best mimic the characteristics of an amputee climbing stairs. Because bilateral amputees lack lower leg muscle, such as the calf muscle, they are unable to provide any spring force from the trailing leg to help propel them onto the next step. To approximate this lack of trailing leg force in the force plate model, the leading foot was placed on the wooden block gently, with the leg bent at approximately $90^{\circ}$. The trailing leg was then lifted vertically, with no "kickoff" force. Once balanced only on the leading 
foot, the leading leg was extended at the slow, medium, and fast rates to isolate the forces generated to the leading leg only.

Force plots were produced from each mode, with five plots each. Plots of the three modes are shown to provide the general force characterization for each mode with respect to time, as seen in Figure 15. The slow step mode shown in Figure 15A shows a relatively smooth ramp-up in force, followed by a plateau. This is most likely due to the slow and smooth extension of the leg, which would not cause any abrupt jumps in force. The medium step mode shown in Figure 15B seems to ramp up quicker than the slow mode, with more variation in force as the time increases. A peak is noticed during the first part of extension, which is possibly a result from producing a quicker motion of extending the leg at a faster rate. After the initial peak, force decreases, and then ramps back up to a more stable force with some variation. This decrease in force could possibly be due to residual upward momentum after the leg is fully extended, therefore effectively lifting some of the body weight off the wooden block. Lastly, the fast step mode shown in Figure $15 \mathrm{C}$ is similar in shape to the medium step mode. However, the initial peak and successive decrease in force are much more pronounced than in the medium step mode. Force is much larger in the fast step mode, which could be explained by more rapid propulsion of the body onto the wooden block, which would require more force downward to propel the body upwards at a quicker rate. The decrease in force, or trough, following the peak could be explained the same as the decrease in the medium step mode, where momentum of the body is continuing vertically after full extension of the leg. The decrease in the fast mode is more substantial, which would be expected since a quicker, larger downward force would provide more momentum in the upward direction than in 
the medium step mode. Small variations in force after an initial peak could be explained by issues of balance on the wooden block.
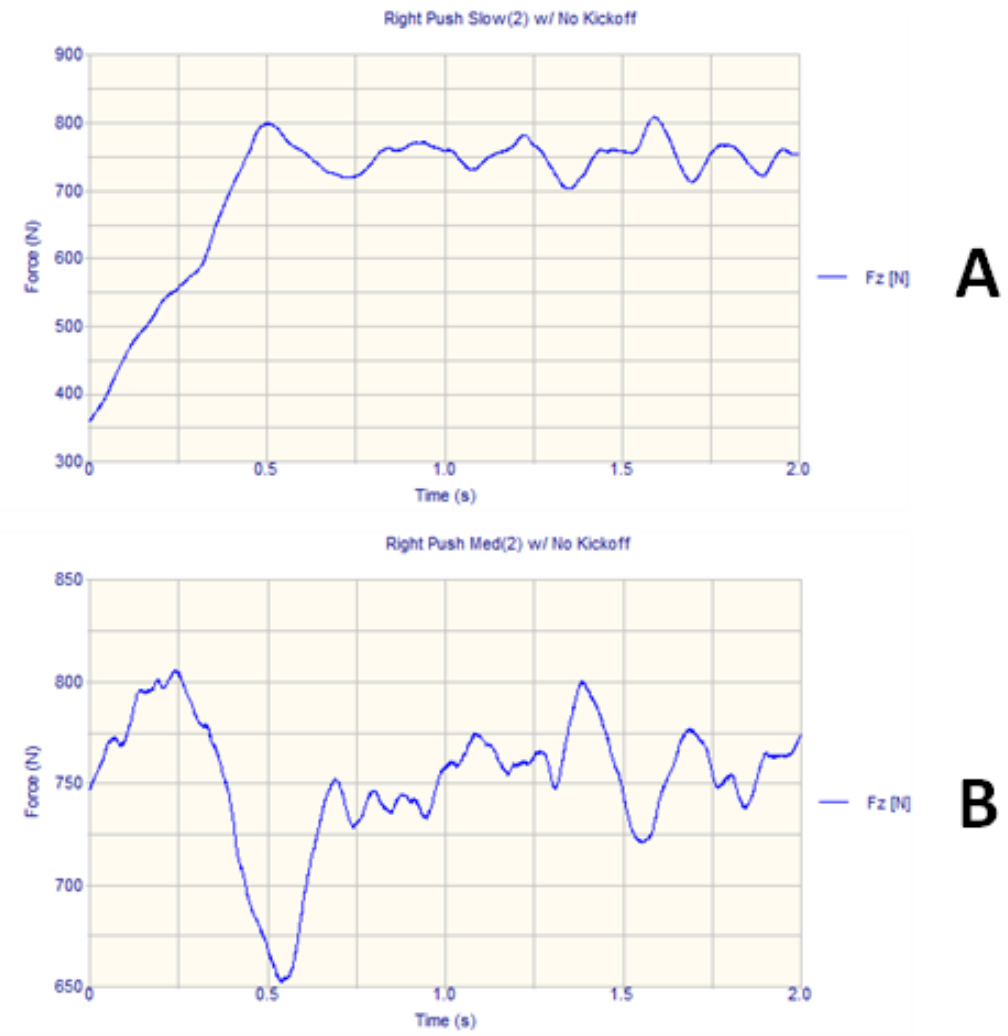

B

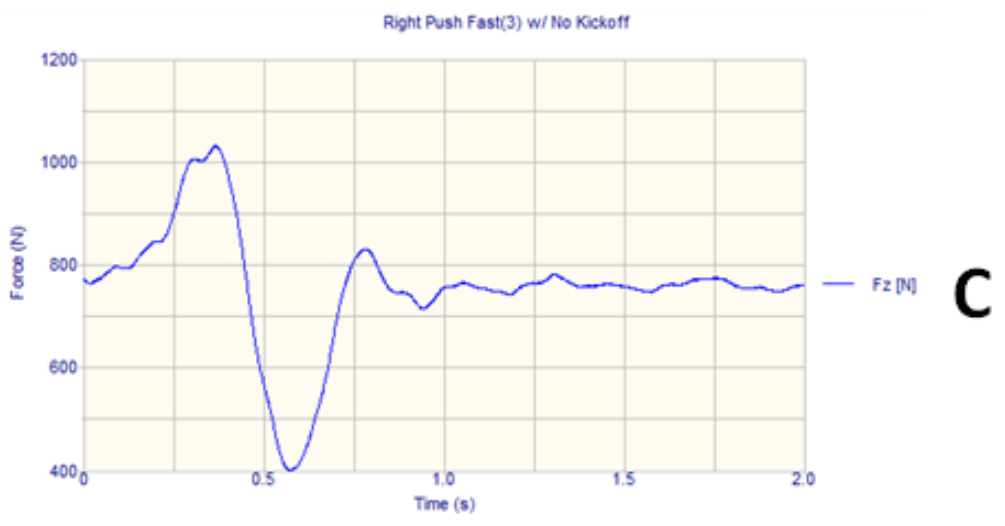

Figure 15: Force plate testing plots were created to characterize force generation in the z-direction with respect to time. One of the five plots from each mode is depicted to show differences in shape and force with respect to time. 
Once testing on the force plate was complete and all plots were created, analysis of the raw data was completed. Maximum forces in the $\mathrm{z}$-direction were noted from each trial for each mode. Averages maximum force for each mode was then calculated, which would be used to calculate torque necessary for stair climbing within the force plate model, as seen in Table II.

Table II: Maximum Forces and Averages from Force Plate Stair Climbing Model

\begin{tabular}{|c|c|c|c|}
\cline { 2 - 4 } \multicolumn{1}{c|}{} & \multicolumn{3}{c|}{ Peak Force Values (N) } \\
\hline Trial \# & Slow & Medium & Fast \\
\hline $\mathbf{1}$ & 825.58 & 817.90 & 1009.39 \\
\hline $\mathbf{2}$ & 808.71 & 805.68 & 968.16 \\
\hline $\mathbf{3}$ & 787.02 & 780.56 & 1032.51 \\
\hline $\mathbf{4}$ & 807.00 & 794.96 & 964.24 \\
\hline $\mathbf{5}$ & 807.75 & 827.47 & 1028.21 \\
\hline Average & $\mathbf{8 0 7 . 2 1}$ & $\mathbf{8 0 5 . 3 1}$ & $\mathbf{1 0 0 0 . 5 0}$ \\
\hline
\end{tabular}

Average values for force generation in the z-direction were very similar in the slow and medium step modes. The medium step mode registered a slightly smaller force production than the slow model, which was unexpected. It was expected that the medium step mode would produce a force greater than the slow mode, but less than the fast mode due to the speed at which the leg was extended, which would translate to the amount of downward force. This unexpected difference in data could be due to tester error, inconsistencies in leg extension speed, force plate error, or other unknown factors.

To estimate the torque that was applied about the knee during force plate testing, length of the test subject's upper leg was measured, and multiplied by the maximum force. With the test subject's upper leg measuring 18", or $0.4572 \mathrm{~m}$, from hip to knee, an average torque of $386.19 \mathrm{~N} \cdot \mathrm{m}$ was created. To normalize this value to the more recent 
literature review, the torque value was divided by the test subject's weight, which was $77.11 \mathrm{~kg}$, yielding an average torque of $4.77 \mathrm{~N} \cdot \mathrm{m} / \mathrm{kg}$. It was noted that this value was over four times as the maximum torque in the literature review. This difference could be present for many reasons including a different test setup, differing sizes and weights of test subjects, errors during force plate testing, using a simplified model, or other factors. Although this force plate model did not agree with either of the previous methods for approximating torque about the knee during stair climbing, it would provide another means for comparing device force data. 


\section{Manufacturing}

Each component of the device was either custom machined, purchased and modified, or used as purchased. Technical drawings of all components that were custom designed and manufactured can be seen in Appendix A. All components were manufactured to design specifications in order to create a fully assembled device, as seen in Figure 16.

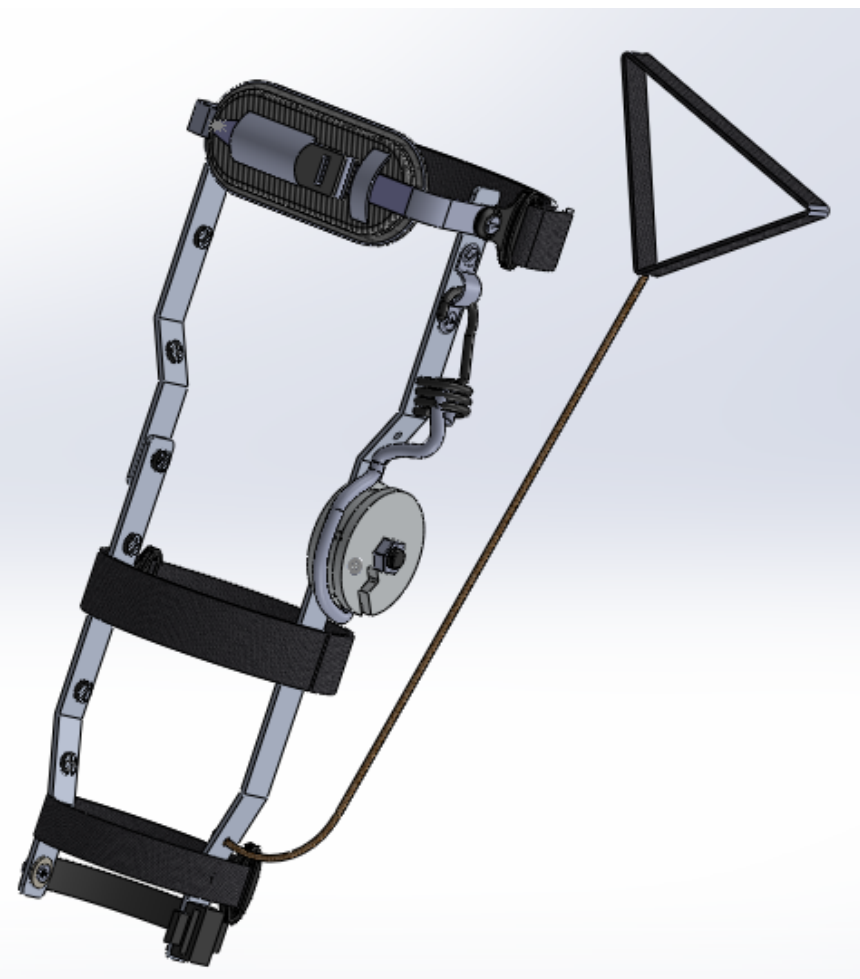

Figure 16: Fully assembled CAD model of device created in Solidworks to ensure proper design and fit of components.

\subsection{Custom Shaft}

The custom shaft for this device was manufactured from a 1/2” X $1 / 2$ " X 12 ” long spring steel standard key stock. The bar was first cut to a smaller size of approximately 2.5 " long in order to allow at least $1 / 2$ " of material for the chuck to grip on the lathe. With 
at least $1 / 2$ " of material being gripped by the chuck, the necessary 0.125 " of square key stock to later engage with the square hole would be guaranteed to remain untouched throughout the lathing process. The newly cut piece of $1 / 2$ " $X^{1 / 2}$ " key stock was then set up in a 4-jaw chuck on a manual lathe. A 4-jaw chuck was used, rather than a universal 3-jaw chuck, due to the original square cross section of the key stock. In order to ensure that the part was centered on the machine so that it would rotate about its true centerline, a dial indicator was used to check deflection at each corner of the key stock while spinning the chuck by hand. Adjustments were made based on deflection values until each corner of the key stock deflected the dial indicator equally.

With the key stock completely centered in the chuck, the lathe was engaged and the end of the key stock was faced off to provide a smooth surface on the outer end. Based on design of the custom shaft, a minimum of 1.6575 " of the shaft would need to be rounded to a $1 / 2$ " diameter, which meant only turning the corners of the square key stock. With the part spinning, a turning tool was used next to turn the specified portion of the $1 / 2$ " $X 1 / 2$ " square key stock into a $1 / 2$ " diameter rounded bar. Using the digital readout on the lathe, the turning tool was zeroed at the corner, as well as the end of the part while spinning to provide reference points of how long and deep the cuts would be. The specified length of the key stock was then turned down to a $1 / 2$ " diameter by taking 0.025 " deep cuts for the majority of the turning operation into the material at a slow feed rate in order to produce a smooth finish. Cutting fluid was used at all times to keep the tool and part cool for a clean cut.

Once closer to the $1 / 2$ " length, subsequent smaller depth of cuts such as 0.010 ", 0.005 ", and 0.001 " were made to avoid removing too much material. The digital readout 
was used to ensure that each pass was the same length, until there was a minimum 1.6575 " length of $1 / 2$ " diameter. Lathing operations were stopped at multiple points to double check dimensions with calipers, and to ensure that the part remained centered. Once the shaft was close to its designed 1/2" diameter, pauses would be taken in the process to attempt fitting of the bearing over the shaft to avoid cutting too much material from the key stock, which would provide a loose fit of the bearing. Once the $1 / 2$ " diameter shank of the custom shaft was tested successfully with the bearing for fit, the end of the shaft was faced off one more time to provide a smooth outer face. After finishing the turning operation, it was noticed that the shaft was slightly off center, which was most likely caused by error during centering in the 4-jaw chuck. However, this did not affect the fit of the bearing, or function in any other way.

With the shank of the custom shaft complete, the part was removed from the chuck and brought to a grinding wheel to grind a small chamfer on the end of the shaft to allow for a simpler and smoother fit of the bearing, as well as to provide a starting point and guide for threads that would be applied at a later time. The shaft was taken to a mill in order to cut a keyway into the shank to lock to the wheel by means of a steel key. The shaft was placed in a vise with a V-block clamping against the rounded portion of the shaft, leaving the square portion hanging off the side of the vise, as depicted in Figure 17. 


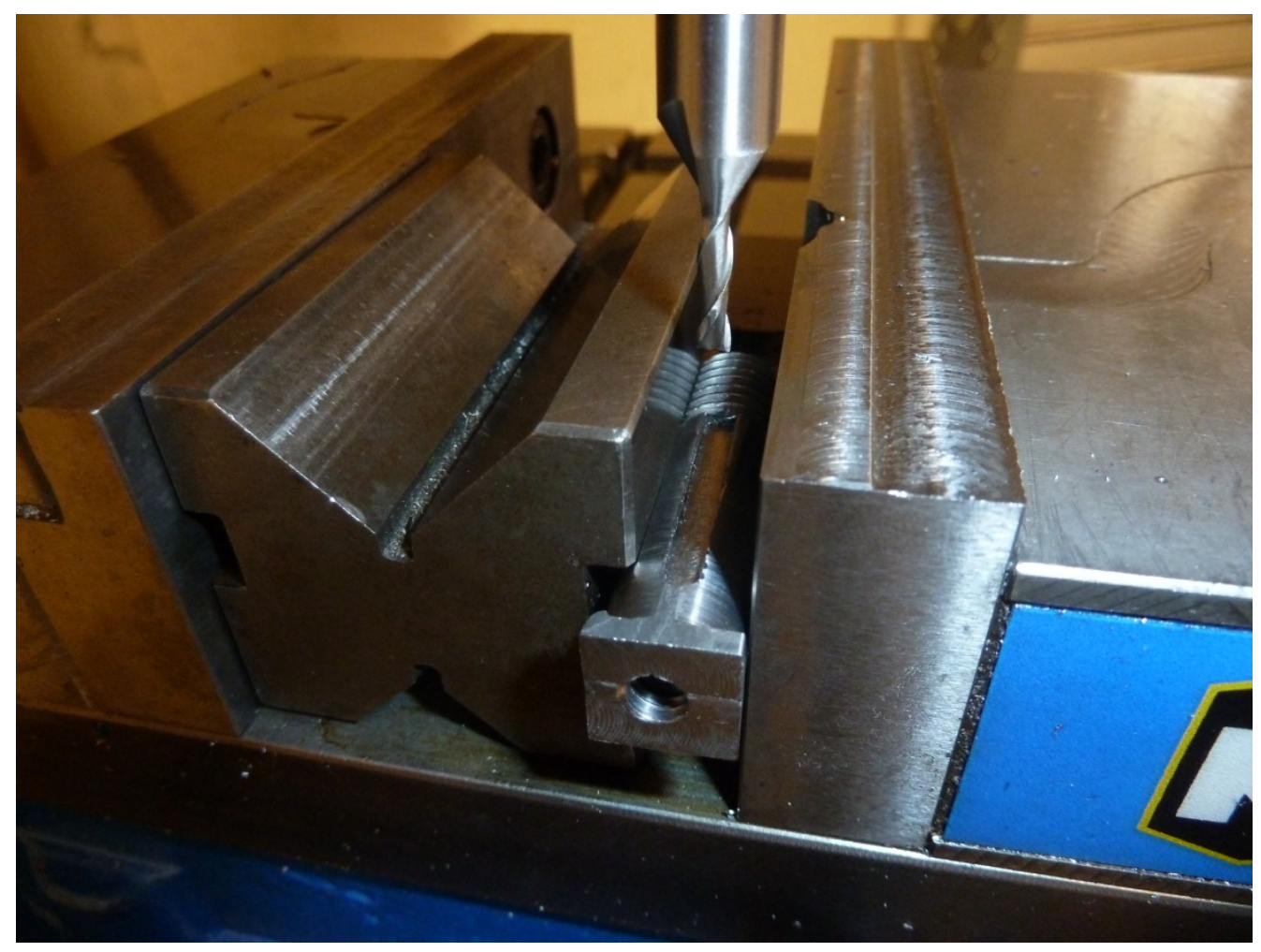

Figure 17: Machining the key way slot in the custom shaft. Setup included the custom shaft being vised with a v-block with the square head hanging off the end so that one of the side faces of the shaft's square head was parallel to the mating vise block.

The surface of the keyway was designed to be parallel with one of the side surfaces of the square portion of the shaft. A dial indicator was used to ensure that the part was level in all directions. A 1/8" 4-flute center cutting end mill was used to cut the keyway in the shaft. Using the digital readout, the end mill was zeroed at the end of the shaft, and measured to the appropriate starting point of the keyway, where it was zeroed again on the $\mathrm{x}$-axis. The end mill was then zeroed on the $\mathrm{y}$-axis by touching the tool to the side of the square portion, then moving in $1 / 4 "+1 / 16$ " for half the diameter of the end mill in order to perfectly zero the center of the end mill. With the x-axis and y-axis both zeroed, the end mill was placed at the $(0,0)$ coordinates, and turned on. The knee of the mill was 
raised to bring the part to the end mill until it first touched, which acted as the z-axis zero point. This point was noted on the knee hand crank manual dial in order to keep track of the depth of cuts. The part was cut by first plunging the end mill into the part 0.015 ", and then fed in the X-axis for the desired length of cut, which was 1.125 " in order to account for the rounds created at the end of the cut where the square cross section key would not fit into. Successive cuts were made until a final depth of $1 / 16$ " was reached, which is half of the key depth. The other 1/16" would be broached into the wheel later so that the key would fit snuggly and therefore lock the wheel and shaft together. Cutting fluid was used during all mill operations.

The shaft was then removed from the vise, and repositioned so that the square portion was on top, with the shank pointing straight down below it. The part was clamped in a vise on both sides of the square portion, and a 3/8" 4-flute end mill was inserted into the mill. The top face of the square portion was milled off in multiple passes of decreasing depth until only $1 / 8$ " of the square portion remained.

In order to cut a hole in the top face of the square head on the shaft, the shank was placed in a universal chuck on a lathe, and an appropriately sized drill bit from a tap set for a $1 / 4$ "-20 tapped hole was used to drill a hole. Once the hole was drilled, threads were created in order to receive a $1 / 4$ "'-20 wide head screw. However, the hole was not drilled deep enough, so modifications to the length of the $1 / 4$ "-20 wide head screws were made in order to fit properly in the tapped hole with the underside of the screw head laying flush against the square head of the shaft. Let it be noted that the hole was not placed back in the lathe and drilled to a deeper depth to correctly receive the screw because the shaft was 
slightly off center, which would have possibly caused an irregularly shaped hole if redrilled.

Lastly, threads were created on the end of the shaft to secure a $1 / 2$ "-13 nut. This was done by placing the custom shaft in a vise, with the sides of the square head flush against the vise grips. A $1 / 2$ "'-13 die was used to cut threads into the end of the shaft. During the process, the vise slightly loosened, causing the shaft to torque within the vise due to the large amount of force from the die turning. Because of this, two of the corners on the square head deformed slightly. The part was re-vised and ensured to be secure before finishing the threads. Threads were cut onto the end of the shaft in order to be sure that the nut would screw onto the shaft and mate with a washer pressed against the wheel.

\subsection{Wheel}

Due to the nature of the previous work using the same wheel design, the exact wheel from the alpha prototype was used for the new prototype, which is shown in Figure 18. Previous manufacturing of the wheel is described to provide the background for the machining and design. The wheel for this device was first designed in Solidworks based on the design dimensions, as well as manufacturability. 


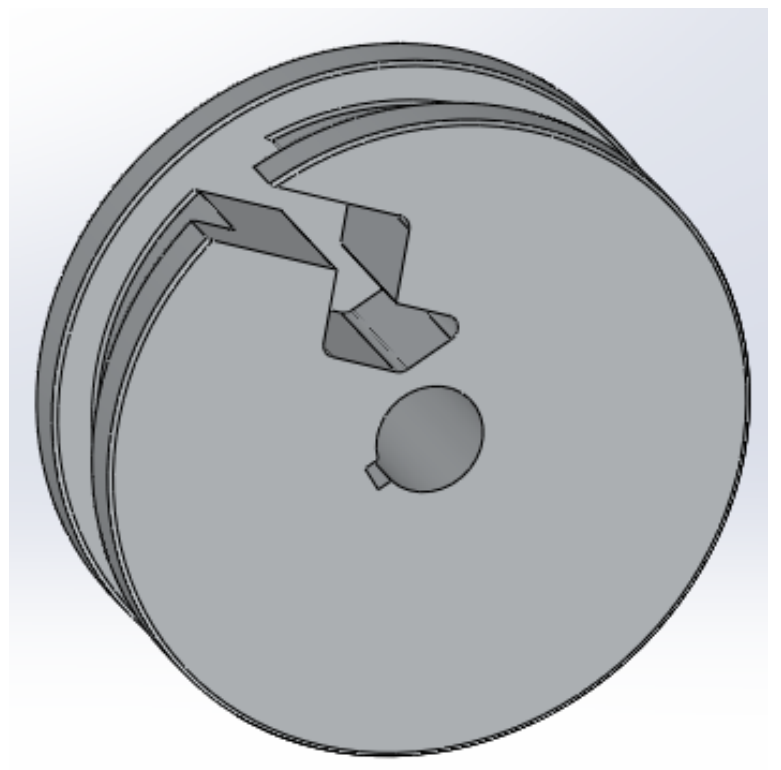

Figure 18: Wheel design from alpha prototype. The exact wheel from the alpha prototype was used for the new prototype. Slight modifications to broach depths were made.

A 4" diameter 6061 aluminum bar stock was cut to a thickness of approximately 1.5", which is $1 / 2$ " thicker than the designed width of the wheel. This extra material was left on the newly cut round in order to provide material for the lathe chuck to grip for initial facing and turning operations. The solid model file was given to the Mustang 60 machine shop, where code was written for $\mathrm{CNC}$ operations on a lathe to face the ends, turn the aluminum round to the designed size, cut the bungee track, cut the shaft hole, and cut all the fillets. The wheel was then put into a CNC mill, where the rest of the Solidworks design, including the zig-zag bungee attachment slot, was machined. Once the finished part was received from the shop, some dimensions were out of specification, including the shaft hole and the zig-zag slot. These were the only two dimensions out of specification that affected the function of the device. In order to remedy these issues, the shaft hole was drilled out to the specified drawing size using a reamer on a drill press 
with the wheel securely fastened to the drill press table. To fix the zig-zag slot, a manual mill was used with a 1/8" 3-flute end mill to cut extra material from the sides of the slot with sharp edges to reach the specified dimensions on the drawing. Multiple setups were required for the modifications on the zig-zag slot in order to access all angles of the slot. Before each cut was made on the zig-zag slot, a dial indicator was used to ensure the part was in line with the axis for cutting. Cutting fluid was used for all operations on the wheel.

In the previous work, the wheel shaft hole was also broached to receive a key. However, the original key was a different size than the newly designed key for the system. This resulted in the need for a newly broached keyway in the wheel's shaft hole. A 1/8" broach with its appropriate $1 / 2$ " bushing was used with a hydraulic arbor press and cutting fluid to broach a new keyway in the wheel, which can be seen in Figure 19. 


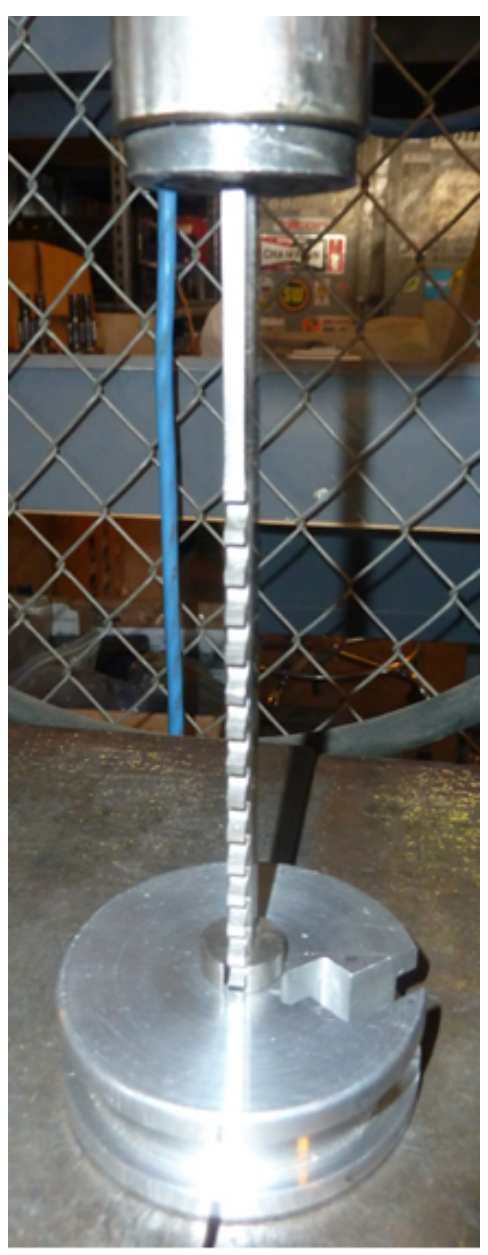

Figure 19: The wheel was broached in a hydraulic press to create the desired keyway

Location of the keyway was designed to place the zig-zag slot opening facing directly down with the brace completely extended. Once the original broach was completed, it was not to the appropriate depth of $1 / 16 "$, so a shim was necessary, as it was in the previous work. However, the shims provided with the broach kit used were not of the appropriate thickness, so a custom shim was made from thin sheet metal cut with metal shears and adhesive tape while measuring thickness with calipers, as seen in Figure 20. 


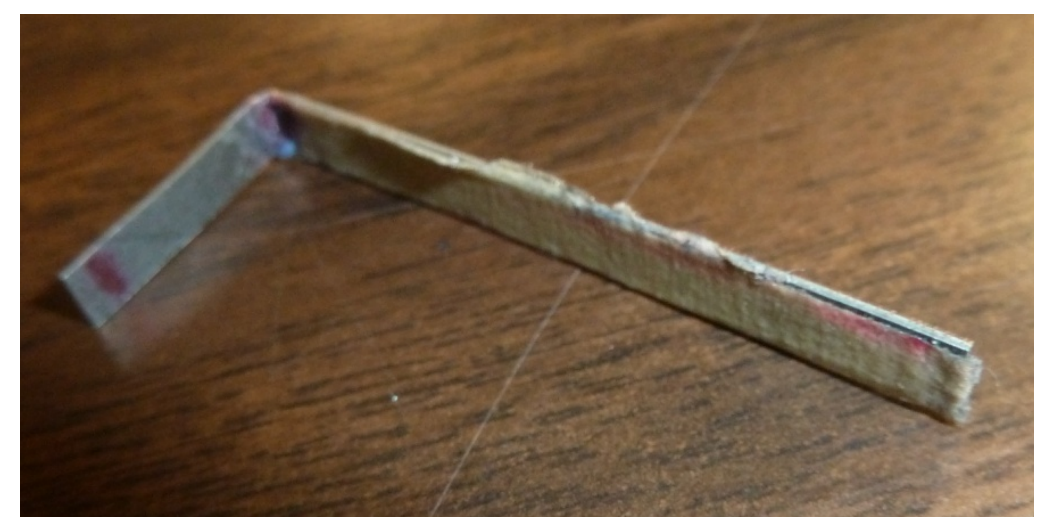

Figure 20: A custom shim was manufactured for broaching the wheel. Additional thickness was added to the shim with tape, and measured with calipers to ensure the proper thickness before completed the broached keyway.

During this process of using the custom shim, the newly broached keyway was accidentally cut to an uneven depth, as well as a depth that was greater than needed. This occurred due to the custom shim slipping into the bushing during the broaching operation because of the cutting fluid and lack of force on the top of the shim. This left the wheel's shaft hole with two unusable keyways for the new key. A third keyway was broached, making sure to provide enough force on the shim so that it did not slip. The third keyway was placed $180^{\circ}$ from the second keyway created, which would provide the correct starting position of the bungee cord as long as the custom shaft was placed in the lower brace's square hole ensuring to line up with the new wheel keyway.

\subsection{Square Hole Broach}

In order to manufacture a square hole to receive and lock the custom designed shaft's square head, a square broach was utilized. A square broach functions similar to a standard broach, however, cuts are made on all faces of the broach. The square broach starts with a cylindrical end to fit in a pre-drilled hole. The cutting faces begin after this 
cylindrical end in an octagonal cross section, slowly transforming into a square cross section to provide the final square hole. It is standard for this process to result in a hole with four sharp corners, with slightly rounded faces, as seen in Figure 21.

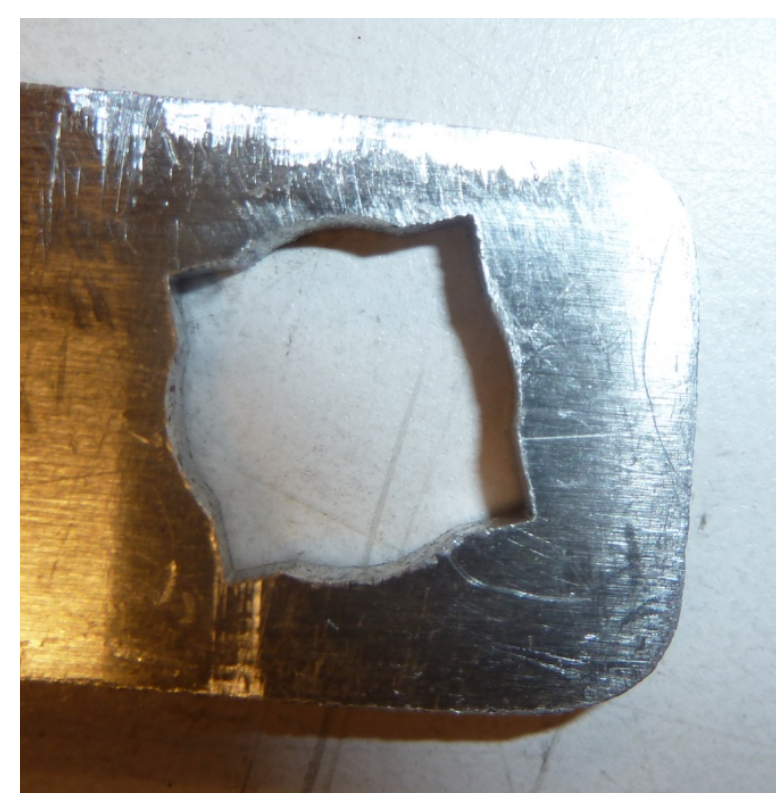

Figure 21: Brace component with the completed square hole.

This occurs due to the fact that the diameter of the required pre-drilled hole is larger than the square portion of the broach. For the square hole created in the lower aluminum band, a $1 \frac{1}{2}$ " square broach was used. In order to receive the $\frac{1 / 2}{2}$ " square broach, a 17/32" hole was first drilled into the lower portion of the top of the lower brace band where the square end of the custom shaft would fit. Two separate $1 / 8$ " thick aluminum pieces also had a 17/32" hole drilled into them. It was necessary to stack three different aluminum pieces on top one another during broaching due to the amount of force applied, as seen in Figure 22 . 


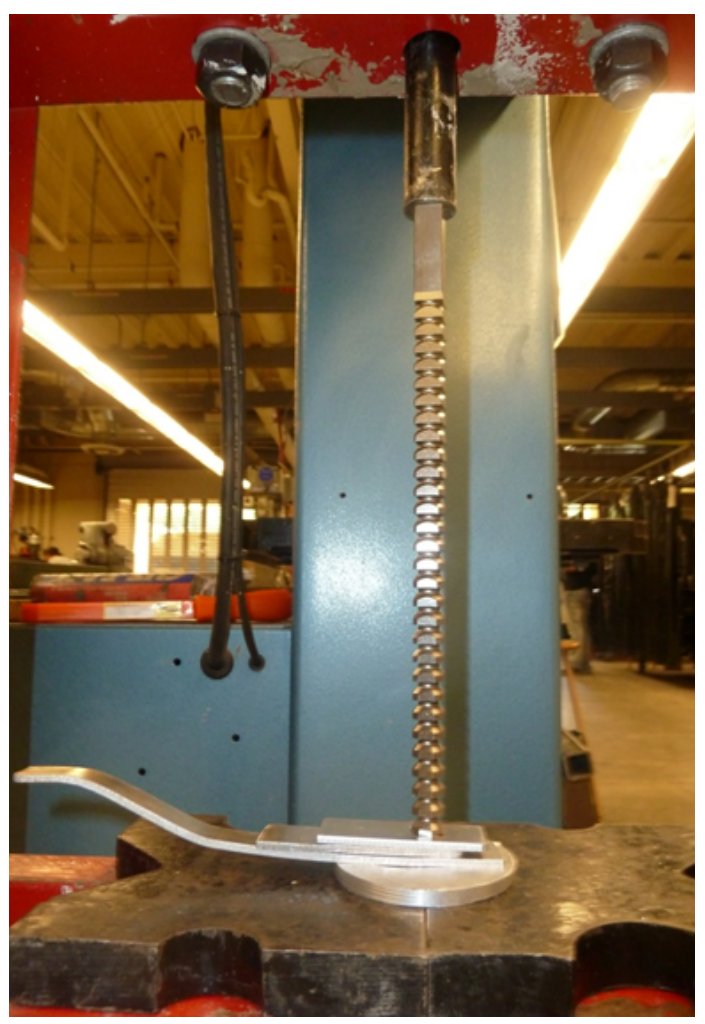

Figure 22: Broaching the square hole through three pieces of aluminum stacked to prevent bending of the brace component

If the material being broached was too thin, it would cause the material to bend.

Therefore, more material was broached in order to keep the desired piece from bending. The square broach was then aligned so that the hole would align correctly with the keyways in the shaft and wheel, resulting with a starting, or fully extended, position of the bungee facing directly downwards toward the ground. Once properly aligned, a hydraulic arbor press was used to push the square broach through the 17/32" hole in the aluminum brace to create a $1 \frac{1}{2}$ " square hole with four tight corners. Cutting lubricant was used while passing the broach through the aluminum to provide a smooth and clean cut. 


\subsection{Brace Components}

The brace components of the device provide the overall structure. All of the brace components, which will be referred to as bands, are 6061-T6 aluminum. Each of the four structural aluminum bands were cut from a sheet of $1 / 8$ " thick aluminum with a camera guided plasma cutter. The camera guided plasma cutter was used because it allowed for a fast and continuous, single cut for each band in roughly any geometry. This plasma cutter uses a camera to follow lines on a piece of paper that is placed on a flat surface adjacent to the actual plasma cutter. The camera is rigged to the cutter in a oneto-one fashion so that the cutter follows the exact same movements as the camera, as depicted in Figure 23.

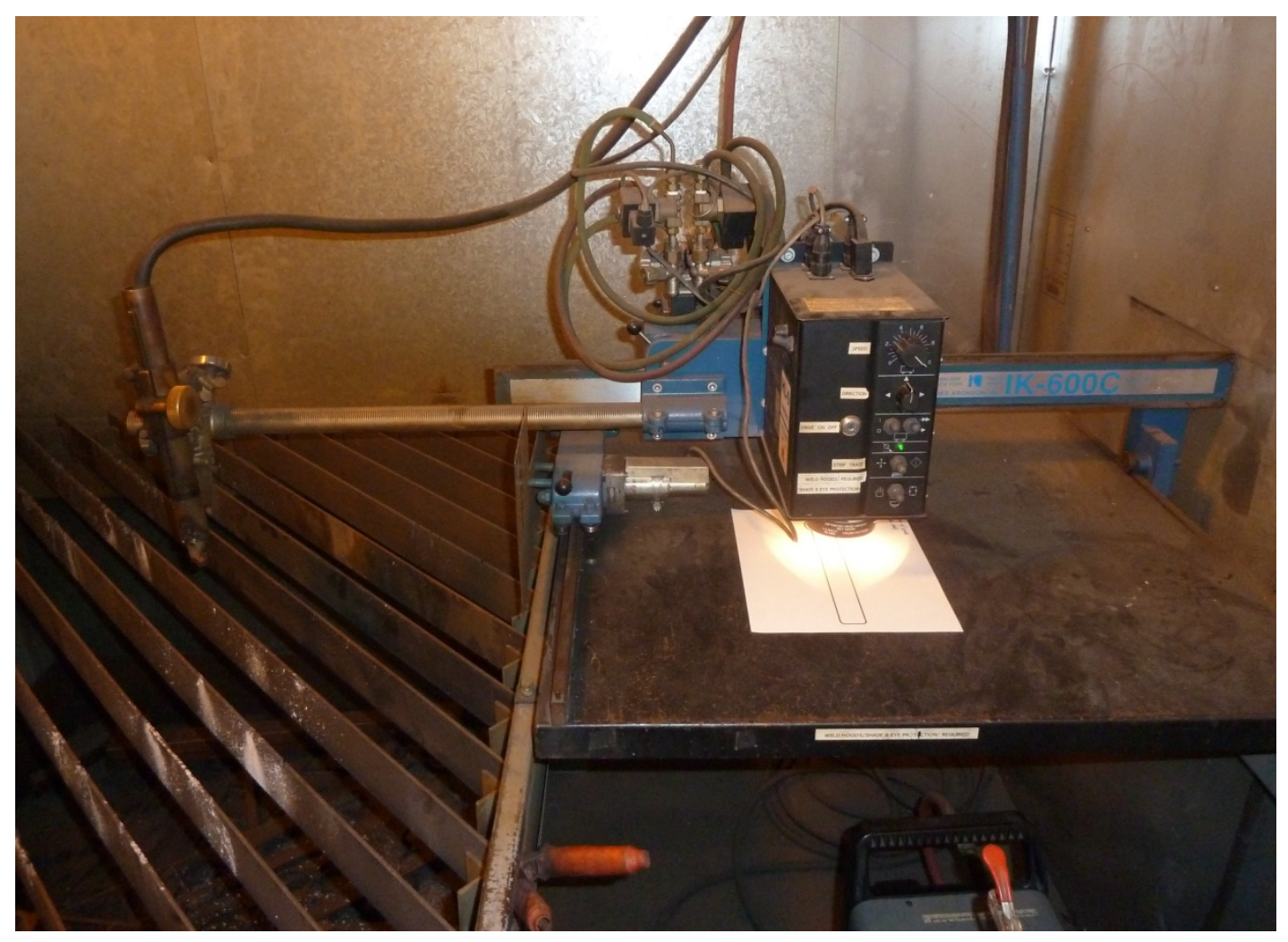

Figure 23: The plasma cutter is set up to cut one of the brace bands. A CAD drawing of the brace band with thick lines is placed directly under the plasma cutter camera. 
In order for the cuts to be made accurately, the bands were first designed in Solidworks to create a full scale drawing of each component. From previous experience, lines on the drawings were made wider, or bolder, than the standard lines created in order for the camera to sufficiently view and follow. If the lines are too thin, they will not be picked up, or tracked, by the camera. This "bolding" of the lines was accomplished within the solid model drawing in order to retain accuracy of the geometry and dimensions of the drawing. When "bolding" the drawing lines in Solidworks, it was noted that the added line thickness was added evenly to both sides of the original lines. With this in mind, as well as the fact that the plasma cutter removes a substantial amount of material during use relative to the width of the brace bands, the drawings created for the plasma cutter were adjusted to be wider in order to compensate for this extra loss of material. This allowed for the brace bands to be within specification of the designed dimensions for proper function and stability. A common problem with the camera guided plasma cutter is that it has trouble tracking along sharp corners in a drawing. To bypass this issue, all areas of the brace bands were designed with relatively large fillets to both help the tracking of the camera and to have rounded corners for safety. After plasma cutting each band, all edges of the bands were sanded to smooth out the roughness created by the plasma cutter.

Once the general shapes were cut for the brace bands, a hole for the bearing needed to be drilled in the upper left band. Because the bearing would be press fitted into the bearing hole, the dimensions would need to be precise and accurate. Using calipers, the exact center point of the hole was marked, and punched with a center punch. A manual mill was used for this operation to ensure a precise and stable cut. The upper left 
brace band was placed in a vise on the mill table, and secured on a flat block of wood, as shown in Figure 24.

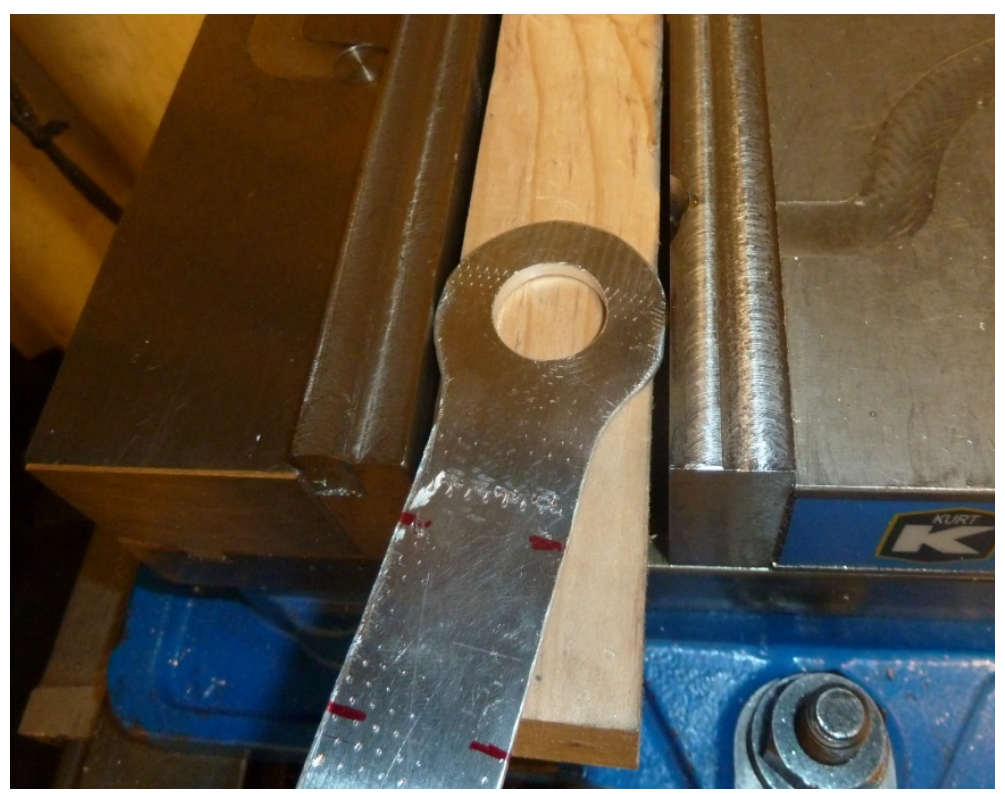

Figure 24: The upper left brace band secured in a vise for drilling the bearing hole.

The vise was tightened with care to ensure that the rounded portion of the band that was being gripped was not deformed to an extent that would compromise function or fit overall. A universal chuck was placed in the mill, rather than a collet, in order to receive a center drill. The center drill was first zeroed at the point of the center punch so that the 3/4" end mill that would be used to drill the hole to size would be able to drill in the center of the hole. A pilot hole was drilled with the center drill. The chuck and center drill were removed from the mill, and a $3 / 4$ " end mill was loaded into the mill with a collet. Ensuring that the $3 / 4$ " end mill was in the correct location, a $3 / 4$ " hole was drilled carefully with cutting fluid applied during the process. Because carbide end mills are typically slightly undersized, this hole would be just under $3 / 4$ ", allowing for the $3 / 4$ " outer diameter bearing to press fit into the hole. 
With the bearing hole successfully drilled, holes for binding posts to fasten different bands were drilled. All hole locations were measured with calipers and marked, then center punched. A \#5, or .2055" diameter, drill bit was used to drill the holes. The brace bands were secured on a drill press table on top of a piece of wood, and drilled at all hole locations. All holes were finished by deburring the edges using a deburring tool.

Lastly, the brace bands needed to be bent into the desired shape. In order to apply a general contoured shape to a prosthetic socket, knee, and lower leg, the profile of an entire prosthetic leg, specifically an adjustable socket with an Össur Total Knee, provided by an amputee was considered. With the original intent for the design of the top of the device to fit around the mid to lower thigh, locations were marked on the edges of the brace bands for bending in order to allow the brace to help follow the contour of an average prosthetic leg. Locations for bending included above the knee joint where the socket tapers down, another point just slightly above the knee joint to allow a flat surface for the upper and lower portions of the brace to assemble with each other in a parallel fashion, and two points at the lower portion of the brace in order to fit to the contour of the pylon or lower leg, as seen in Figure 25.

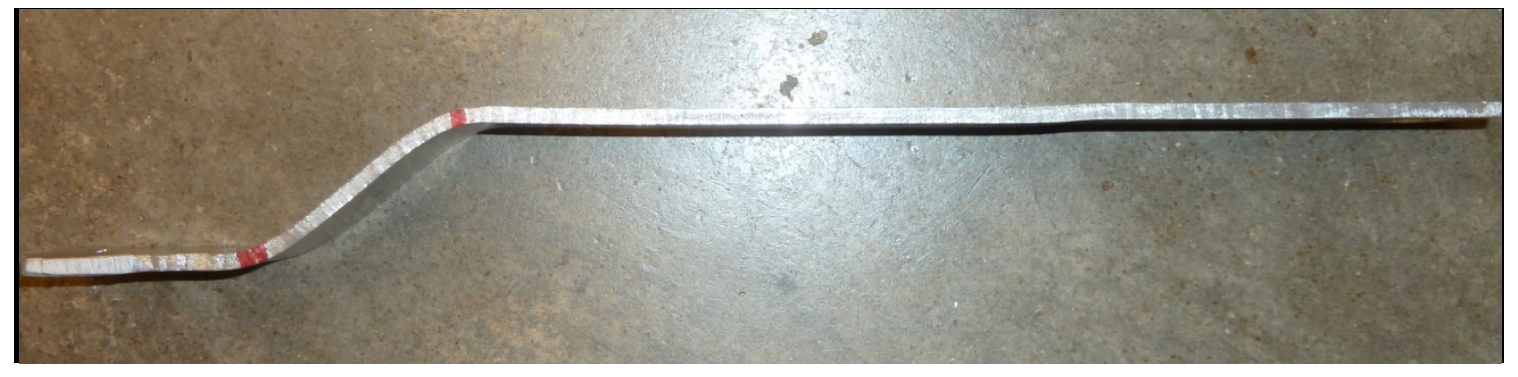

Figure 25: One of the lower brace bands depicting the finished product of marking and bending to provide the general contour of the prosthesis. 
The locations for bending on the upper left aluminum band were also dimensioned to ensure that the wheel would not come into contact with the brace after assembly.

Because all sockets are generally unique to each patient, as well as there being a large amount of variability in width and height of prosthetic knees, angles of the bends were not precisely calculated, but were bent to specifically follow the contour of the prosthetic leg used for sizing. Although this was not an exact process, a general contour was all that was desired since the adjustability of the brace would compensate for any differences in prosthetic sizes. Figure 26 shows how each aluminum band was gripped in a vise and bent in small increments by hand at the marked locations.
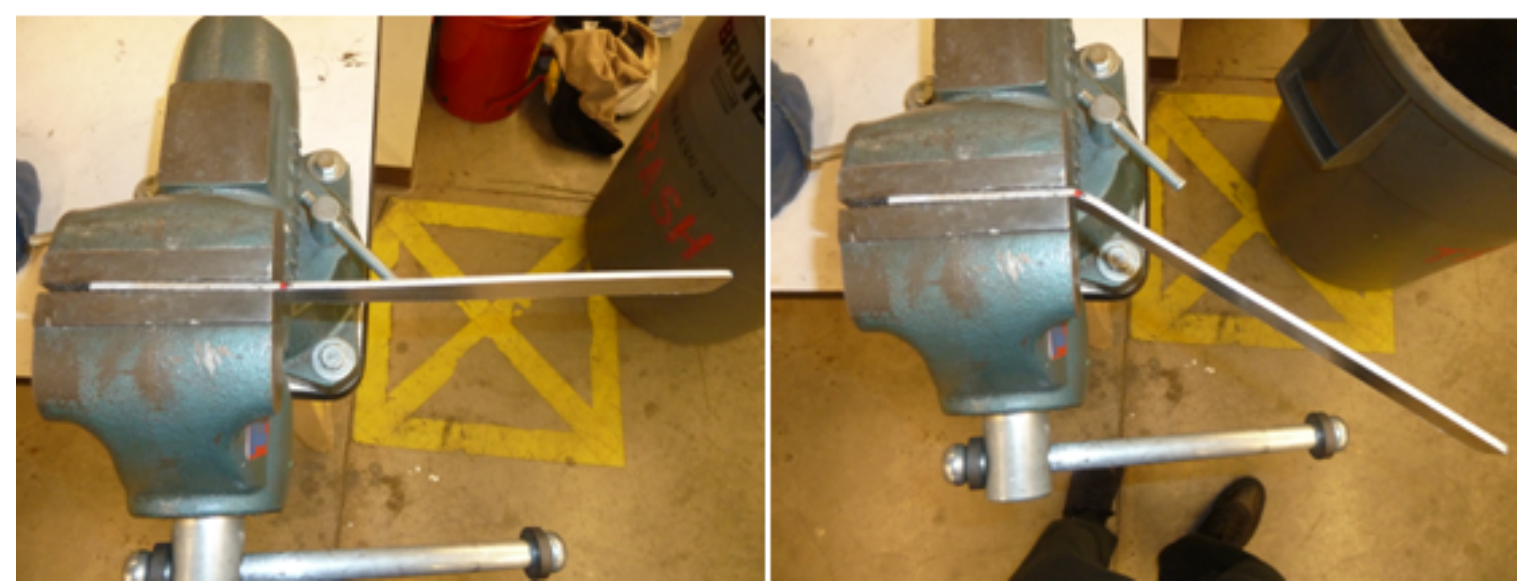

Figure 26: Before and after photos of bending of a brace band. The image on the left shows the band marked with red ink at the ends of the vise, and the image on the right shows the band bent at the marked location.

The bands were removed from the vise throughout each successive bend to check the angles, ensuring to follow the contour of the prosthesis. Because the design of the brace was to have the left and right brace bands identical, the two sides were bent to match one another in both length and angles of the bends. 


\subsection{Adjustable and Velcro Straps}

The adjustable straps and Velcro straps incorporated into the device were either purchased or taken from a donated orthotic brace. Both of the adjustable straps were purchased from m2inc., and all other Velcro straps and plastic loops were taken from a donated brace. A larger, padded adjustable ratcheting strap was used for the top of the brace to secure the front of the thigh. A Velcro strap was also placed directly behind the upper adjustable padded strap to successfully encompass the entire socket. To secure the lower leg to the brace, a smaller and more basic adjustable ladder strap with a receptor was used to loop around the back end of the lower leg. Because the lower ladder strap was relatively stiff and would not hold a hemispherical shape, modifications were made to the strap itself. The strap was heated over a stove burner until it was pliable, curved into a shape that would successfully fit a prosthetic lower limb, and quenched in cold water to hold the shape. Both the ladder strap and receptor were secured to the lowest point of the brace. A Velcro strap was secured directly above the lower ladder strap and receptor to hold the lower leg to the brace. A single plastic loop was used, and the Velcro strap was wrapped completely around the leg and brace, allowing it to grip on itself to secure the leg to the brace. The one plastic loop method with the Velcro strap looped around the entire brace was used twice more, with one location slightly below the knee joint, and one slightly above in order to more securely attach the brace to the prosthesis. An additional Velcro strap was added between the upper adjustable strap and middle Velcro strap to provide a more secure fit, which can be seen in Figure 1. 


\subsection{Padding}

Adhesive bumper pads were used for padding throughout the inside of the brace. These pads were used to cushion and grip against the prosthetic. This would help prevent from scratching or slipping while in direct contact with surfaces on the prosthesis. Round, $1 / 2$ " diameter X 1/8" thick, adhesive bumpers were used because they secure well to the aluminum frame, grip well to most surfaces, and apply enough cushion to help form fit the device to the amputee. Multiple bumpers, rather than a couple longer continuous pads, placed throughout the aluminum bands provide enough cushion and protection while keeping weight down. Each adhesive bumper pad was placed approximately $1.5 "$ to 2 " from one another to provide enough padding. Adhesive bumper pads were also specifically placed adjacent to all binding posts and on top of the binding post at the knee joint.

\subsection{Pull Handle}

The pull handle for the system was made by using paracord securely tied to a padded handle that was borrowed from an elastic resistance band. The paracord was then tied directly to a hole drilled in the brace to provide an attachment point.

\subsection{Bungee Attachment}

The bungee attachment used was a purchased component. A handrail bracket package was purchased from a local home improvement store, from which only the rounded bracket in the package was used. Modifications to the bracket holes were made since they were too small for the binding posts. The bracket holes were drilled out with a \#5 drill bit to achieve a size that would allow them to receive the binding posts. The holes were deburred using a deburring tool. 


\subsection{Assembly}

The device was assembled with multiple binding posts of differing sizes, nylon washers, one jam nut, and small amounts of Loctite in specific locations. All components were assembled per design to create the final, functioning prototype, as seen in Figure 1. Before any main components of the device were assembled, the bearing was press fit into the bearing hole on the upper left brace component using a rubber mallet. The lower adjustable strap and receptor were then secured to the brace with binding posts so that the ladder strap wrapped around the back side of the brace with the smooth surface facing the inside. Loctite was used in the binding posts at these locations to keep the adjustable strap from swiveling up or down, which would cause the binding posts to come loose. The larger size binding post was used for the receptor, with a nylon washer on the inside of the brace to take up any extra slop. The lower Velcro strap was then secured to the brace with a single binding post just above the adjustable strap in the appropriate binding post hole drilled in the brace. Both of the center Velcro straps were also secured to the brace with a single binding post as well. The upper padded adjustable strap was secured in the same location as the upper Velcro strap, with the adjustable strap across the front, and the Velcro strap across the back. The upper padded strap ends were placed over the binding post holes, and the two plastic loops for the Velcro strap were placed directly on top of the adjustable strap ends so that the holes were all concentric. The longer sized binding posts were used on both sides of the brace to secure both straps to the brace. A washer was placed between the upper and lower portion of the brace at the knee joint on the right, directly lateral from where the wheel would be installed. With the upper brace knee joint hole on the outside, the lower brace knee joint hole on the inside, and the nylon 
washer between the two, a shorter length binding post was inserted and fastened with Loctite to secure the upper and lower brace together. The bungee attachment point was then mounted to the upper left brace component with the smaller binding posts. Nylon washers were placed between the binding posts and the inside of the brace to take up slop. All binding posts were secured with the flat head slot facing the exterior of the brace for any necessary adjustments.

With the frame of the brace and straps assembled, the wheel assembly was assembled next. The custom shaft was first inserted from the inside of the brace, passing the threaded end of the shaft through the square hole in the lower brace component, through a thin nylon washer, and finally through the bearing. The shaft was twisted until the corners of the square head aligned with the square hole so that the keyway on the shaft faced directly towards the back of the brace. With the shaft correctly positioned, the low profile wide diameter head screw was screwed into the tapped hole on shaft's square head, with two thin nylon washers between the square head and the underside of the screw head. Another thin nylon washer was slid over the shaft, and pressed up against the bearing. The key was placed in the shaft's keyway, and the wheel was slid onto the shaft with the bungee zig-zag slot facing outward so that the key fit in the newly broached keyway in the wheel with the zig-zag slot facing directly down. With the wheel secured in place against the thin nylon washer, a thicker nylon washer was slid over the shaft, and pressed against the outside of the wheel. Lastly, a jam nut was screwed onto the threads of the shaft, ensuring that the nut was tight enough to hold the wheel, shaft, and brace assembly together without any slop. 
The round, adhesive backed bumpers were then placed along the inside of the brace to provide sufficient padding to prevent from harming the prosthesis, as well as to help form fit the brace to the prosthesis. Lastly, one end of the paracord was tied to the metal loop in the padded handle from the elastic resistance band, and the other end was tied to a hole in the lower left brace with bowline knots. 


\section{Testing}

Testing of the new prototype was conducted in multiple stages to assess general design, fit, and functionality. A bench top test was completed to assess the force output from the bungee cord energy storage system. Once bench top testing was complete, the device was shipped to WRNMMC for testing with amputees to evaluate the overall performance.

\subsection{Bench Top Testing}

Bench top testing was done with the completed new prototype to gain data on force generation. Although the energy storage system of the new prototype device was almost identical to the alpha prototype, bench testing was conducted to ensure that there were not any minor altercations that would drastically alter the force output. The testing model was set up identical to the alpha prototype testing, using a table top vise, the device, paracord, and weights. The bungee was inserted into the wheel's zig-zag slot, and the hook was connected to the bungee attachment bracket. With the lower portion of the brace was placed in the vise with an aluminum block to provide a flat surface since the brace component was bent, weights were hung from a binding post hole in the upper portion of the brace with paracord. Beginning with a $3 \mathrm{lb}$ weight and adding weights until a final weight of $16 \mathrm{lbs}$ or $20 \mathrm{lbs}$, angle of the knee joint flexion at each weight was recorded. With the angle of flexion corresponding to the applicable weights and the distance from the knee joint to the point of force, moments were calculated about the knee joint for each weight tested. This process was repeated for a 6", 7", and 8" bungee of $1 / 2$ " diameter to provide a range of data that was compared against one another with a plot. 


\subsection{Testing with Amputees}

Testing with amputees was conducted to gain more tangible data and feedback on the device's overall performance with respect to its intended use. Due to the lack of amputees available in the local area, testing was outsourced to WRNMMC. Three amputees of varying attributes tested the device for its performance in providing an assist for stair climbing. These attributes including sex, height, weight, prosthesis model, and whether they are a unilateral or bilateral amputee were all noted to help evaluate the feedback provided by each amputee, as seen in Table III.

Table III: Attributes of test subjects

\begin{tabular}{|c|c|c|c|c|c|c|}
\hline $\begin{array}{c}\text { Test } \\
\text { Subject } \\
\#\end{array}$ & Sex & Height & Weight & $\begin{array}{c}\text { Prosthesis } \\
\text { Model }\end{array}$ & $\begin{array}{c}\text { Mechanical or } \\
\text { Microprocessor }\end{array}$ & $\begin{array}{c}\text { Unilateral } \\
\text { or } \\
\text { Bilateral }\end{array}$ \\
\hline $\mathbf{1}$ & Male & $5^{\prime} 10^{\prime \prime}$ & 175 & Total Knee & Mechanical & Bilateral \\
\hline $\mathbf{2}$ & Male & $6^{\prime} 2^{\prime \prime}$ & 185 & Rheo Knee & Microprocessor & Bilateral \\
\hline $\mathbf{3}$ & Male & $6^{\prime} 0^{\prime \prime}$ & 220 & X-2 & Microprocessor & Unilateral \\
\hline
\end{tabular}

Testing comprised of adjusting the device and attaching it to the patient's prosthesis, as well as attempting to climb stairs with the device in its active setting. All testing was completed with staff on standby for safety. Figure 27 depicts the evaluation form that was provided to the amputees to assess general attributes, fit, and function of the device after testing was completed. 


\section{Evaluation of Stair Assist Device for AK Amputees}
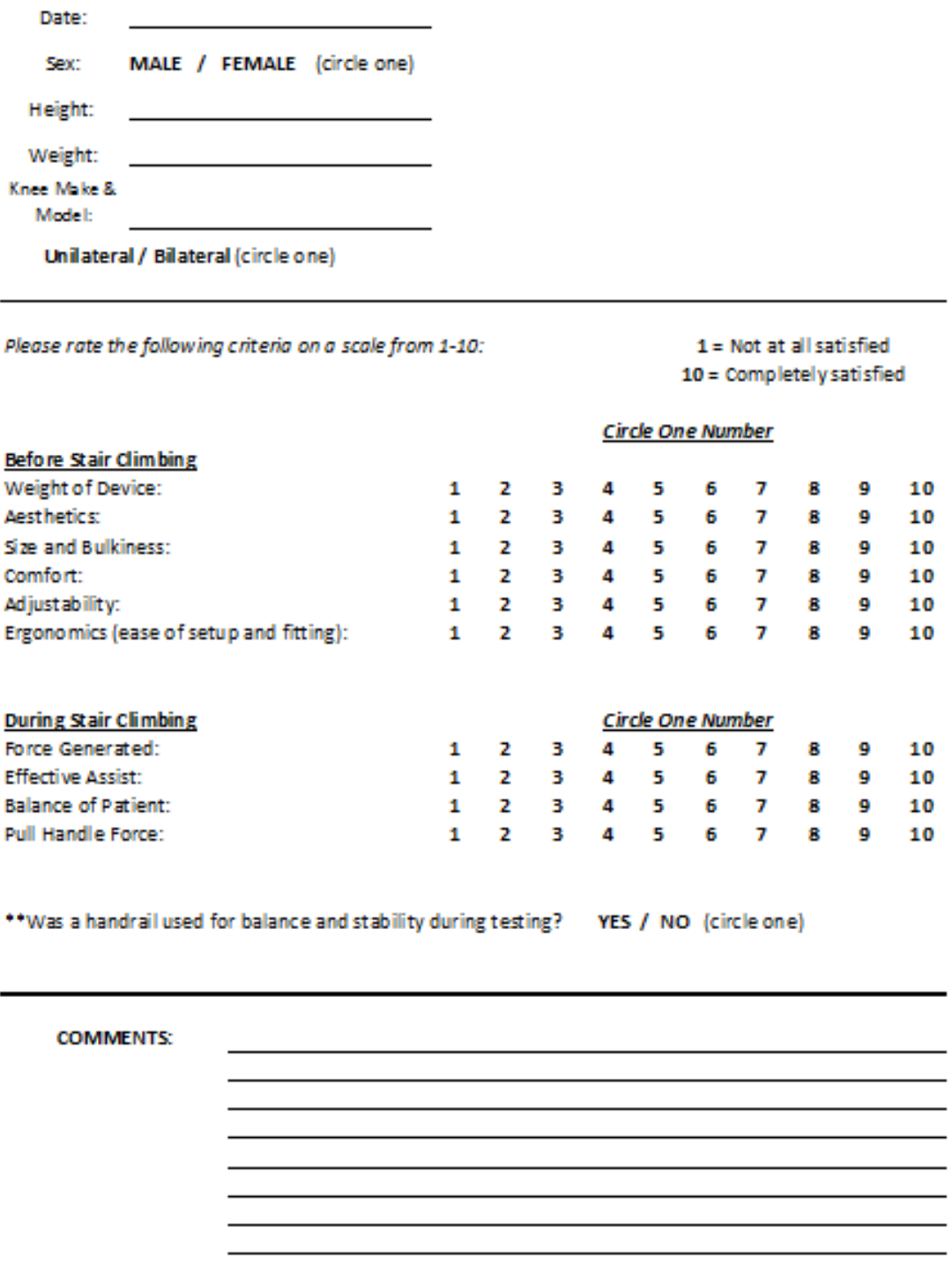

Figure 27: A testing evaluation form provided for amputees at WRNMMC to rate separate categories of general attributes, fit, and functionality before and during testing. 


\subsubsection{Background}

As mentioned previously, a lack of local amputees meant that testing would need to be outsourced. With contacts at WRNMMC provided by QL+, it was possible to ship the completed prototype to the orthotics and prosthetics division at WRNMMC, located in Maryland. The prosthetic and orthotic service at WRNMMC offers care to a large amount of amputees, providing a perfect test population for this stair assist device. A minimum of three amputees for testing was requested with the specification that there be at least one bilateral and unilateral patient, as well as at least one mechanical and microprocessor controlled prosthesis. This allowed for a relatively full range of testing for the different types of patients and prostheses. Amputees would provide feedback by filling out an evaluation form with attributes of feedback split into categories of "Before Stair Climbing" and "During Stair Climbing". The attributes were divided into these categories to provide partial feedback on the device prior to testing the functionality in order to get the amputees' general impression of the device before active use.

\subsubsection{Fitting and General Attributes}

General fit of the device would be assessed based on the evaluation form feedback from the amputees. In the area of general attributes and fit, the evaluation form provided a scaling system from 1 to 10 , with 1 signifying "not at all satisfied" and 10 signifying "completely satisfied", for the criteria of weight, aesthetics, size and bulkiness, comfort, adjustability, and ergonomics. These criteria were compiled in a category on the evaluation form titled "Before Stair Climbing". The feedback gained from this portion of the test would come from the amputee visually inspecting the device, attaching the device themselves, and carrying out standard walking and stair climbing motions. 


\subsubsection{Function}

Overall function of the brace would be assessed based on the "During Stair Climbing" portion of the previously mentioned evaluation form, as well as video footage captured during testing. Qualitative data would be gathered from the evaluation on the device's performance as far as function and force generation was concerned, and the video footage would be analyzed to produce quantitative data of actual force generation during actual use of the device. The evaluation form criteria for qualitative data based on function was rated on the same scale, from 1 to 10, as the "Before Stair Climbing" criteria. The criteria in this category included force generated, effective assist, balance of patient, and pull handle force. These specific criteria would provide a general sense of how well the device function during stair climbing, while also providing feedback on secondary issues such as balance and force required for the pull handle, while noting whether or not a handrail was used for extra stability and balance during testing.

Quantitative data would be gathered from the video footage captured during testing. With the video footage provided from a side angle with the outer face of the wheel facing directly to the camera, moments about the knee would be calculated based on the initial flexion angle before release of the pull handle, and the time taken to extend the leg to a locked position. These two factors would provide enough information to calculate the moment about the knee joint on a rough scale. Although the video footage method would not be the most accurate vehicle for gathering quantitative data, it was the only option seeing as the device acts as an "assist" rather than providing the full force to complete the stair ascent. There are also many factors that could play a role in skewing the data from the video footage such as momentum carried throughout the stair climbing 
process, balance issues, an unsteady or off angle camera, and friction or resistance within the prostheses. With these possible issues taken into account, it was concluded that video footage was the only option for gathering quantitative data, especially since the testing was outsourced, and the designer could not be present. 


\section{Results}

\subsection{Bench Top Analysis}

Bench top testing of the new adjustable prototype provided quantitative data on the performance of force generation. This bench model was set up in the same manner as all previous bench top testing, with the device secured in a vise and weights hanging from the device itself. Moments were calculated based on the amount of weight hung from the device, the perpendicular distance of the force to the knee joint, and the angle of flexion at equilibrium. Maximum moments of $3.63 \mathrm{~N} \cdot \mathrm{m}, 3.17 \mathrm{~N} \cdot \mathrm{m}$, and $2.51 \mathrm{~N} \cdot \mathrm{m}$ were calculated for the 6", 7", and 8" bungees respectively. Although these values are slightly larger than the alpha prototype bench top testing moments, they are relatively similar, which was expected since the design of the bungee attachment and energy storage mechanism was almost identical. A plot of torque generation was created to compare the torque values at differing angles of flexion for all lengths of bungee used, as seen in Figure 28. After points were plotted, curves were fitted to the data to provide a general shape of the bungee's force generation with respect to degree of flexion, which are represented by solid lines in Figure 28. 


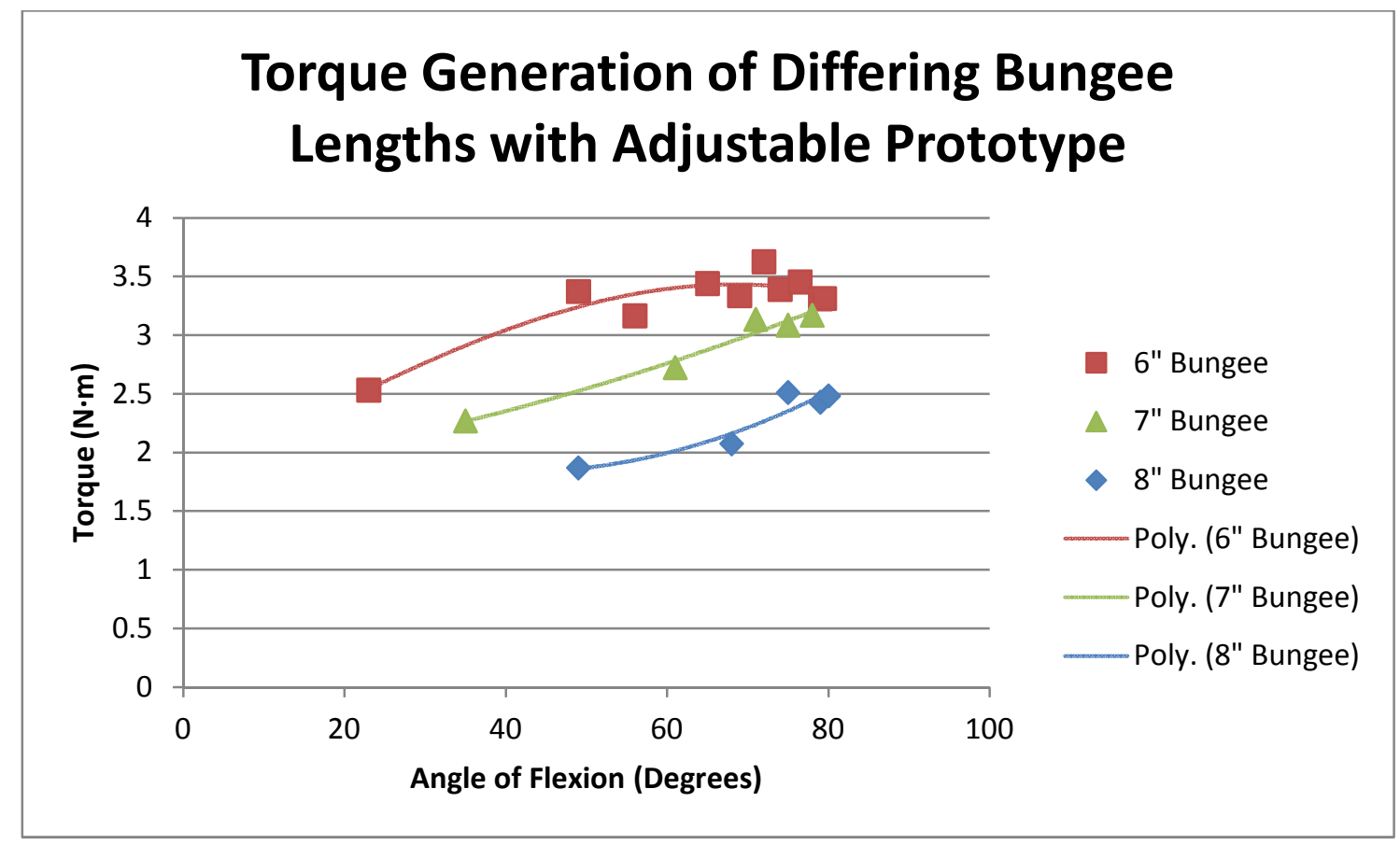

Figure 278: Torque generation of three different length bungee cords from a bench top test with the completed new adjustable prototype.

General shape of the fitted curves differed between the bench top testing results from the alpha prototype and the adjustable prototype, as shown in Figure 8 and Figure 28. The fitted curves on the alpha prototype plot seem to climb, reach a peak, and descend back down, while the fitted curves on the adjustable prototype plot are more characteristic of a slightly curved, but almost linear, line that consistently increases until the final points. The exact reason for these differences in curve shape are unknown. However, one possibility for differences could be due to a slightly different length of the bungee attachment points, which would cause differences in the pre-stretched condition of the bungee. Other reasons that the plots don't match could be minor differences in setup of the test, testing error, friction caused by mechanical slop within the system, or error during the process of recording data. 
Forces generated by the bungee cords were then used to calculate the percent assist according to previous literature. Using the largest value of torque, which was provided with the 6 " length bungee, along with the average weight and torque of patients in the previous literature review, a $6.14 \%$ active assist was calculated. Although this assist seems minimal, it can slightly vary based on weight of the amputee. For example, if using the minimum weight of $62.6 \mathrm{~kg}$, which is found in the previous literature review, an assist of 7.48\% is provided with the 6" bungee (Vallabhajosula, Yentes, \& Stergiou, 2012). Although it seemed that this assist was possibly not adequate for stair climbing, testing results from actual amputees would need to be analyzed and evaluated to accurately describe the success of the device.

\subsection{Amputee Testing Analysis}

Testing with amputees at WRNMMC provided valuable feedback to help gauge the success and shortcomings of the device. Completed evaluation forms were returned, along with comments and general feedback. Although qualitative and quantitative data were expected from testing, quantitative data was not able to be obtained.

Video footage of the testing would have allowed for the moment about the knee joint to be calculated, therefore providing an approximated value for torque provided during actual use. Unfortunately, video footage of the testing with amputees was not captured as requested. With no video footage, quantitative data could not be produced for the testing at WRNMMC. Quantitative results for the device could only be provided from the bench top testing as described above.

One form of qualitative data was the ratings from the amputees that completed the evaluation forms, which can be seen in Appendix B. A total of ten categories in the 
"Before Stair Climbing" section and four categories in the "During Stair Climbing" section were rated from one to ten, as described previously. These ratings were then averaged for each category to provide a general sense of satisfaction of the device, as seen in Table IV.

Table IV: Average ratings for qualitative categories of testing the completed device on amputees while climbing stairs

\begin{tabular}{|c|c|}
\hline Before Stair Climbing & Average Rating \\
\hline Weight of Device & 6 \\
\hline Aesthetics & 5 \\
\hline Size and Bulkiness & 4.7 \\
\hline Comfort & 4.3 \\
\hline Adjustability & 5 \\
\hline Ergonomics (ease of setup and fitting) & 4.7 \\
\hline During Stair Climbing & Average Rating \\
\hline Force Generated & 3 \\
\hline Effective Assist & 2.7 \\
\hline Balance of Patient & 2.3 \\
\hline Pull Handle Force & 3 \\
\hline $\begin{array}{l}1=\text { Not at all satisfied } \\
10=\text { Completely satisfied }\end{array}$ & \\
\hline
\end{tabular}

Overall weight of the device, which is $1.90 \mathrm{lbs}$, proved to be acceptable with an average rating of six, which was the best rated category of all. Generally, the "Before Stair Climbing" categories were rated in the middle, suggesting that they were sufficient, but not completely satisfactory. The "During Stair Climbing" categories, however, had very poor ratings between 2.3 and 3.0. Based on the qualitative rating system, the amputees at WRNMMC seemed to not be satisfied in multiple categories. The evaluation form 
provided great qualitative data to draw conclusions from in a more general sense. However, more detailed qualitative data was obtained in the comments section and general feedback in emails.

Comments included on the evaluation forms addressed problems such as the device slipping down the leg during use, incompatibility issues with certain prostheses, diminished balance, and lack of force generation. One amputee commented that the device would slowly slide down his prosthesis while climbing stairs, making the motion more difficult than it normally is without an assistive device. It was also noted that the device is not useful with microprocessor controlled knees unless they are set in a free swing mode. All amputees experienced balance issues due to the entire motion of using the device, as well as having one hand occupied during use. Lastly, two of the three amputees mentioned that the device did not provide sufficient force generation to actively assist in propelling them up stairs. The amputees that tested the device agreed that the device was useful in some aspects, but would overall provide more of a hindrance and distraction with stair climbing than without the device.

Although there were many comments addressing areas that the device lacked in helping climb stairs, there was positive feedback as well. One amputee with a Rheo Knee noted that the device helped keep his toe up, therefore better preparing the foot before each successive step. The test subject with the Total Knee mentioned that the device helped assist during normal, level ambulation.

General feedback from the contact at WRNMMC who assisted in testing with the amputees was positive, negative, and suggestive of other possible applications. According to feedback, fabrication of the device was outstanding, with no issues in the 
function or with the previous problems such as shaft pull out, slop, and adjustability. It was noted that the adjustability of the device made it much more useful than the alpha prototype, but that the upper adjustable strap could use a non-slip surface. Negative feedback included that the device decreased balance during use on stairs, the lift handle was not detachable, force generation was not sufficient, and that it impaired the function of microprocessor controlled legs more than it helped. Based on testing with multiple amputees with input from staff at WRNMMC, it was suggested that the device may be better suited for the application of a below the knee amputee, patients with chronic nerve deficit of the leg, patients with significant soft tissue loss of the leg, or patients with a significant knee injury. 


\section{Discussion and Conclusions}

\subsection{Concept}

The overall concept of this device proves to be unsatisfactory based on testing and feedback. Although many of the design requirements were met, the design was lacking in areas that were not included in the original requirements originally shown in Table I. The concept for an external attachable stair assist device for transfemoral amputees does seem to be feasible based on testing and feedback from this device, however, modifications or a complete re-design would be necessary to fully satisfy the needs of the amputees. According to the original design requirements, testing results, and feedback, the device performed satisfactorily in the following parameters: weight, noise level, durability, inexpensive, user force input. Some of the other parameters included in the original design requirements were not quite measurable, but proved to be less than satisfactory based on feedback from amputees. As previously mentioned, testing was conducted outside of the presence of the designer, which caused an inability to provide explanations, demonstrations, or general guidance about the device and its use. It is possible that qualitative scores for each section of the device could have been higher if proper guidance could have been offered throughout the testing process. Although the overall design was not a success, it is clear that the concept is successful, and could provide a substantial starting point for a more heavily modified or re-designed device.

\subsection{Fit}

Fitting of the device seemed to be successful in general. In comparison to the previous alpha prototype with rigid aluminum bands that curved around the thigh and pylon, the new adjustable prototype was much more effective in fitting multiple amputees 
with different prostheses. With the category of size and bulkiness rated at an average of 4.7, it seems that the amputees were not quite satisfied with the bulkiness, but were not completely dissatisfied or opposed to the size. One possible reason this rating is on the lower end of the spectrum is that the amputees are not accustomed to having anything external strapped to their prosthesis. Any attachment at all would most likely feel unnecessarily bulky and change the weight distribution of the prosthesis. Another possibility for the lower rating on size is that the test amputees were amputated relatively recently, that is to say they are not completely comfortable or familiar with their prostheses alone. With this in mind, attaching any sort of device would most likely seem like a bulky addition.

An average rating of 4.3 for comfort of the device seems to be relatively low as well. However, upon more detailed review of the qualitative results from the evaluation forms, one amputee rated the comfort of the device as a 6 , which would be relatively satisfactory. On the other hand, another amputee rated the comfort as a 3. This variability in comfort could come from many aspects of the device such as overall length with respect to the amputee's prosthesis length, how well the device was attached, or location of amputation with respect to the comfort of tightening the device on a socket versus a portion of the amputee's natural leg. Complaints of the device slipping down the prosthesis during use were noted, which could have played a role in the relatively low rating of comfort. This issue of sliding was most likely due to the lack of an effective non-slip surface on the straps and pads of the brace. Overall, it seems that comfort of the device seems to be very subjective between amputees, and could possibly be a result of other qualities of the device such as adjustability, weight, size, and general contour. 
Adjustability of the new prototype was relatively successful. The ratcheting straps allowed for the overall width of the brace to adjust to a range of sizes for sockets, knees, and pylons or lower leg portions. The multiple Velcro straps also allowed for adjustability within the overall contour of the brace to provide a more secure and formed fit at four points on the prosthesis. An average rating of 5 for adjustability provided feedback that the amputees felt the device was able to adjust well enough to fit their different prostheses, but did lack in some aspects. Little detail was given as to the ratings for adjustability, but it is likely that the mid-range ratings for adjustability could have been a result of the ease of adjustability, a stiff lower adjustable strap, lack of rigidity due to the independent brace components, or other reasons that were not noted.

One issue that was noticed prior to testing was that the lower plastic adjustable strap was very stiff. As noted previously, treatment of the strap was conducted by heating and quenching to obtain the curved shape necessary to cradle the lower portion of the prosthesis. Although treatment resulted in the desired curved shape, there was relatively no flex in the adjustable strap, which caused slight offsets between the two sides of the brace when widening or narrowing the lower adjustable portion. A more compliant strap would have solved this issue, but was not incorporated due to time constraints with ordering, receiving, and testing. Also, the independent components of the brace reduced overall rigidity, making it more difficult to adjust unless already attached to the prosthesis. In general, the adjustability of the device was a vast improvement on the previous alpha prototype, but was still not completely satisfactory according to test amputees. 


\subsection{Functionality}

Testing with a bench top model and with amputees at WRNMMC yielded valuable feedback about the device and its overall functionality. Bench top testing provided data of force that should be generated during use that would assist amputees in stair climbing. Unfortunately, due to the nature of the device being used as an assist, there was no way to accurately gauge exactly how much force would be necessary to effectively help propel an amputee up stairs. Limitations of the bench top model resulted in quantitative data that does not characterize the performance of the device accurately in comparison to quantitative data that would be obtained through testing with amputees. These limitations included incorporating the natural weight of the prosthesis, compensations from the body such as momentum during walking and stair ascent, forces from existing muscles, and changes in balance during use. While the bench top model did provide quantitative data, the true results and feedback on functionality of the device came from the amputee testing. Based on both models of testing, the device was fully functional in the sense that it worked as designed, and provided torque about the knee joint to assist in stair climbing. The device functioned properly with no malfunctions, and was structurally sound. The previous issues with shaft pull out, mechanical slop, and adjustability were all fixed with the new design, therefore allowing the amputees to test a fully functional product. Although the device functioned properly, it did not perform well enough to provide adequate assist in stair climbing to make the device worth using.

Testing with amputees provided data and feedback that explain the shortcomings of the device, as well as its successes. While quantitative data was planned to be calculated from video footage, there was no video footage provided from testing, which 
eliminated the possibility to produce any quantitative data from testing with amputees. With no quantitative data, results of functionality were solely based on evaluation ratings, comments, and general feedback from amputees and staff at WRNMMC. Functionality of the device was assessed on the evaluation form in the categories of force generated, effective assist, balance of patient, and pull handle force. All these categories combined provide an overall sense of how well the device performed, as well as how well the process for using the device worked. With an average rating of 3 for the amount of force generated, the device was clearly lacking in the ability to provide sufficient torque. All three test subjects rated this category as a 3, clearly expressing its shortcomings in force generation. It was noted that the two amputees with microprocessor controlled prostheses could only effectively use the device while in free-swing mode, which would allow the prosthesis to function similar to a mechanical prosthesis. Effective assist of the device had an average rating of 2.7, which reveals that the overall effectiveness of the device was marginal. Based on comments from amputees and staff at WRNMMC, this low rating was a result of the lack in force generation combined with issues created in balance during use. Average balance of the patient during use was rated a 2.3, which was the lowest rating of any category. The use of a pull handle was incorporated in order to give the user full control of the system, therefore creating a more safe and controllable device. From the initial automated prototype, it was noticed that there are many areas for falters, failures, or bugs within a fully automated design, which could potentially result in a device that is more dangerous than it is helpful since a stumble or misstep during ascent of stairs could end in the patient falling and being harmed. However, after testing with amputees, it is clear that losing the use of one hand to the pull handle creates a substantial 
loss in balance. It was also noted that the general motion and process of using the device was more complicated and distracting than desired, which proved to make the process of stair climbing more difficult. Pull handle force was rated at an average of 3, which was most likely tied in with the issue of balance. While the force required to bend the brace and store energy in the bungee was not substantial in any way, it did provide enough distraction and complications in balance to deserve a low rating. One extraneous factor that might have played a role in the lower ratings is the lack of time spent with the device. Amputees did not have sufficient time to become familiar and comfortable with the device before testing. If more time had been given for the overall testing process and the amputees were more comfortable with the device, there may have been a greater possibility for higher ratings.

As stated previously, there was no accurate way to gauge the amount of assist necessary to help in climbing stairs, especially since each patient would need a different amount of assist based upon many factors such as remaining muscle, weight, height, coordination, experience, or whether the amputee was a unilateral or bilateral amputee. While the lack of force generation was a fault in the design, it was not a noticeable fault due to the fact that the only vehicle for gaining this data was testing with amputees, which were not readily available in the earlier stages of design. The lack of force generation stems from the length of the bungee cord used. The 6" bungee cord provided the most force, which was still not sufficient to adequately assist in stair climbing to the point where it was worth using the device, according to the test subjects. It was understood that a shorter bungee would provide more force; however, a 6" bungee was the shortest possible bungee that could be provided with a hook due to manufacturing 
limitations from the supplier. With a shorter bungee length, more force could be provided, which could possibly be achieved by taking up more of the bungee within the grips built into the wheel, finding a supplier that can produce shorter bungees, or placing the bungee attachment bracket at a higher location on the brace. Although these options could possibly help with providing more force, the maximum stretch of the bungee must be considered. While the bungee can stretch to store energy, there is a point at which the bungee will no longer stretch, therefore putting a limit on force generation. If a shorter bungee would not provide enough force before reaching its maximum stretch, other options for force generation would have to be considered.

While the ratings for functionality of the brace were relatively low, there were some positive comments within the feedback. The bilateral amputee using the Rheo Knee commented that while the device did not necessarily provide enough assist in the means of force generation, it did provide an assist in keeping his toe up, which prevented him from coming into unwanted contact with the front face of each stair during ascent. The patient using the Total Knee also commented that the assist during stair climbing was marginal, but that it did help noticeably during standard, level ambulation. With these positive comments, it was noted that while the device was not quite successful in the realm of stair climbing, it could have potential benefits in other applications.

Staff at WRNMMC also provided general feedback from the overall testing experience. The overall relative ineffectiveness of the device was noted, but suggestions for other applications were made as described previously. Suggestions for alternate applications of the device included using it for below the knee amputees, patients with significant nerve or tissue damage and loss, or patients with significant knee injuries. 
The multiple areas of dissatisfaction culminated to agree with these suggestions. While the device was beneficial in some aspects, the small areas of effectiveness are not enough to successfully promote the device for an assist in stair climbing. 


\section{Future Work}

\subsection{Minor Alterations}

Some minor changes could be made to the device to address a few of the issues presented during testing. First, it was requested that the pull handle be detachable so that it would not be left hanging while the device was not engaged for active use. A solution to this request could be attaching a bracket that is similar or identical to the bungee attachment bracket currently on the brace. The paracord would be attached to a trigger snap seen in Figure 29, which would easily hook onto the bracket. A trigger snap would provide a quick attachment and release of the pull handle.

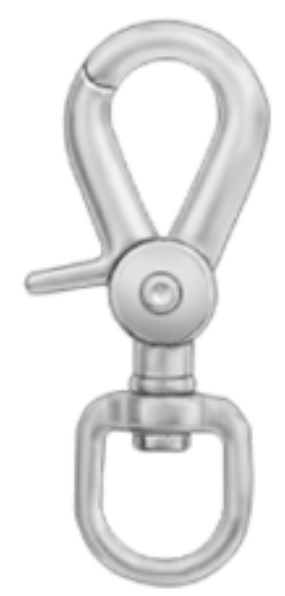

Figure 29: Trigger snap that could be used to allow the user to quickly release the pull handle from the brace if desired (McMaster-Carr).

It was also noted that the device, specifically at the upper padded ratcheting strap, slipped down the socket of the amputee during testing. This issue could be addressed by adding a non-slip surface to the inner surfaces of all pads, Velcro straps, and adjustable straps. Products that could possibly be used as a non-slip surface could be anti-slip tape, anti-slip glue, or an anti-slip paint coating. 
The lower adjustable strap should be replaced with a more compliant material. An elastomer would work well for this application since there are not large amounts of stress placed on the strap, and adjustability is easily attainable due to the many options available on the market for straps made of materials such as rubber.

In terms of data collection, a lack of data points was encountered during this project. Using a larger testing sample for bench top testing would benefit the data by providing a more accurate description of the trends for each bungee. Also, with a larger sample size of data points, statistical analysis could be included to account for any error. Another area of data is testing with amputees. Only three amputees were tested at WRNMMC, which does not provide enough data to be certain of any conclusions. Increasing both the amount of amputees and time tested would create more data to analyze for results.

\subsection{Current Manufacturing and Design Changes}

During manufacturing and testing, it was noted that certain components of the device could be altered, or designed differently to allow for assembly and function with more ease. Machining of the custom shaft proved difficult, as well as time consuming. If this device were to be commercialized, a more streamlined method for creating a shaft would be necessary. As explained previously, the main reason for machining a custom shaft was because commercially available elevator and carriage bolts would not accurately fit within the bearing for smooth rotation. However, for future work, a custom bearing could be ordered, or designed if necessary, to fit a $3 / 8$ " elevator bolt shank. A 3/8" steel elevator bolt, the largest commercially available size, should be capable of upholding to the forces to which it would be subjected. If a properly sized bearing was 
purchased or designed, the only machining necessary for the shaft would be the keyway slot. This new design would sufficiently address all previous issues of shaft pull-out and mechanical slop with the low profile wide bolt head and square neck. Using an elevator bolt with a new bearing would save large amounts of time, which translates to large monetary savings.

Another area for improvement could be the design of the bungee securing system on the wheel. Although the zig-zag slot provides adequate friction to secure the bungee in place for use, it is difficult to properly fit the bungee into the slot. The zig-zag slot also proves to be cumbersome with any sort of tension while the bungee is facing directly outward from the opening, and not biting on the outermost sharp edge. This motion causes the bungee to slide out of the slot, and pulls the sheath off the bungee slightly, which can cause difficulties in re-insertion of the bungee. A change in the design of the wheel could remedy this issue. One design solution could be creating a wheel that is based on two components. While the majority of the wheel would be exactly the same, the zig-zag slot would be straight, a portion of the wheel would be cut out with a dove tail style track, and a second component would slide over the dove tail track and be secured against the bungee by a screw mechanism, as seen in Figure 30 . 

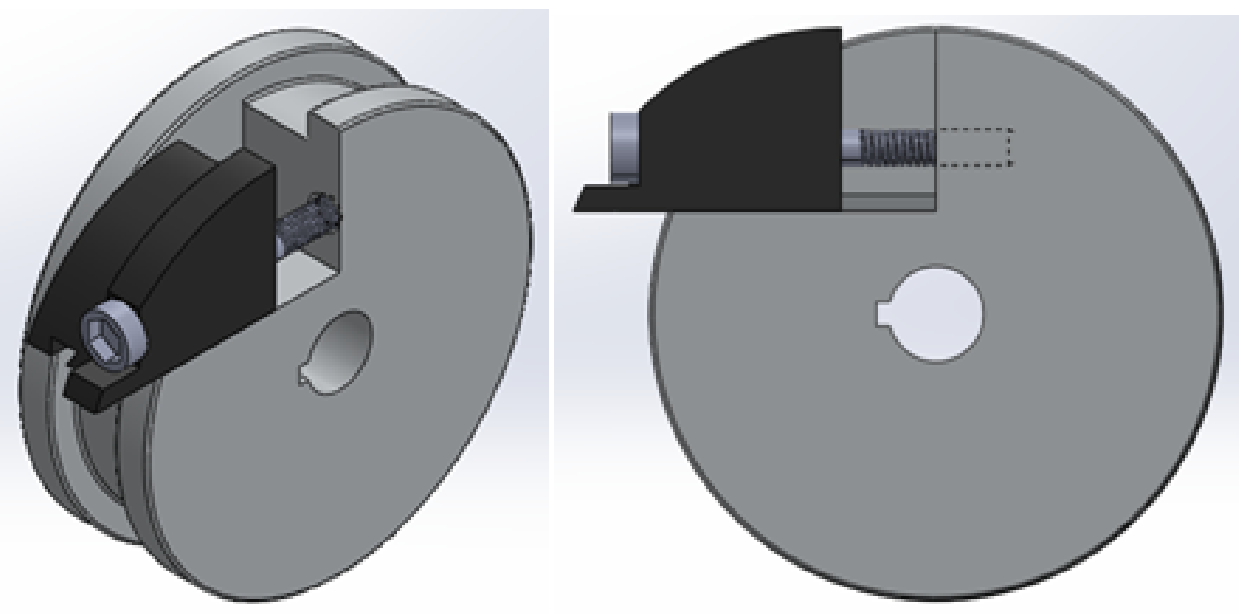

Figure 280: New design for bungee insertion and attachment to the wheel. The black component is the sliding component that is moved in or out to attach or release the bungee by adjusting the bolt.

The sliding component would be screwed in and bind against the bungee tightly, pushing the bungee against its mating surface. Both faces that would mate with the bungee would be rough in finish to provide as much friction as possible. This new two-component design for the wheel would allow the bungee to be easily inserted and removed, while still providing enough force and friction to keep it secured at any angle of tension.

\subsection{Addressing Critical Caveats}

Testing feedback proved that there are major caveats of the device and its use. The first of these is the issue of balance and coordination. Feedback from amputees stressed that using the device greatly diminished their balance during stair climbing. This was attributed to the necessity of using one hand for the pull cord, as well as the actual movement of pulling the handle of the pull cord. It is clear that balance is one of the largest problems created by this device, and needs to be remedied in future work. Options to make the device hands free have been explored previously, such as using a 
harness around the upper arm, which would allow a shoulder shrug to move the device. However, the previous concepts for a hands free, fully mechanical device were neglected for various reasons. Other concepts could be explored to create a hands free, fully mechanical device. If explored, it would be beneficial to research natural movement during stair climbing, as to reduce the amount of unnatural movements that could cause diminished balance during use. Another option to remedy the balance issue is moving towards an automated design again. However, automation of the device would necessitate some sort of force generation that is not produced by the user. This possible solution brings back issues of weight from a motor, piston, or other means of force production. With components such as these, the device would require electronics and software code to control the system. With a completely automated system, there is more opportunity for malfunction or failure than in a simple mechanical design. Malfunction of the device during use could be catastrophic, which proves to be an issue with automating the device, as seen in previous work.

If the proposed changes could be made, the issue of overall force generation would need to be assessed. Feedback from testing suggested that there was a lack of force generation to successfully assist in stair climbing. If the future work was based on a mechanical design, special or custom bungees could be researched to provide more force than standard commercially available bungees. Previous work resulted in abandoning options such as springs, both linear and torsional, due to size and force generation. However, more research could be done in this area, with the possibility of purchasing a special or custom spring. With a spring design, the wheel would be replaced with a gear, much like that on a bicycle. The spring could be welded or 
mechanically attached to a bicycle chain, which would be locked onto the gear. The movement of the device would be very similar, but energy storage would be in a spring rather than a bungee. Another option would be to research and test new materials rather than using a bungee. Using a solid elastomer could provide enough force generation, depending on the material's properties. A solid elastomer would rid of the outer sheath on the bungee which proved to be cumbersome.

While challenges were faced with the limitations described above, there were also challenges within the management of contacts and amputees. Due to the lack of local amputees, almost all of the testing was outsourced to WRNMMC. In future work, it would be beneficial to keep closer contact with local prosthetists and amputees as well as WRNMMC to provide more opportunities for testing to gain feedback throughout the design and testing process.

Overall, the device would need more work in the form of modifications or redesign to provide a successful product. The above options could be researched, while keeping design requirements and user needs in mind. 


\section{REFERENCES}

Amazon supply. (n.d.). Retrieved May 14, 2013, from

http://www.amazonsupply.com/zinc-plated-steel-elevator-length/dp/B00137AGPI

Austen, I. (2002, January 3). A Leg With a Mind of Its Own. Retrieved April 14, 2013, from http://www.nytimes.com/2002/01/03/technology/a-leg-with-a-mind-of-itsown.html?pagewanted $=$ all $\&$ src $=$ pm

Barbarino, C., Glaeser, K., Mensing, R., \& Titchenal, M. (2012). Prosthetic knee leg lift: A device to aid above-knee amputees in stair climbing. Senior Project, San Luis Obispo.

Budynas, R. G., Nisbett, K. J., \& Edward, J. (2011). Shigley's mechanical engineering design. New York: McGraw-Hill.

Component parts limited. (n.d.). Retrieved May 14, 2013, from http://componentparts.co.uk/Elevator\%20Bolt.htm

Ethier, C. R., \& Simmons, C. A. (2007). Introductory biomechanics: From cells to organisms. Toronto, Canada: Cambridge Texts.

History of prosthetics \& orthotics. (2008, July 1). Retrieved April 12, 2013, from http://www.aopa.org.au/index.php?option=com_content\&view=article\&id=71:his tory-of-prosthetics-a-orthotics \&catid=37: general $\&$ Itemid $=58$

Huang, C. e. (1979). Amputation: Energy cost of ambulation. Archives of Physical Medicine and Rehabilitation, 60:18-24.

McMaster-Carr. (n.d.). Retrieved May 14, 2013, from http://www.mcmaster.com/\#trigger-snaps/=mslnpg

Össur. (n.d.). Rheo Knee product specificaitons. Retrieved April 14, 2013, from http://www.ossur.com/?PageID=15744

Össur. (n.d.). Total Knee 2000. Retrieved April 14, 2013, from http://www.ossur.com/?PageID=13446

Ottobock. (n.d.). C-Leg microprocessor prosthetic knee. Retrieved April 14, 2013, from http://www.ottobockknees.com/knee-family/c-leg-microprocessor-prostheticknee/ 
Ottobock. (n.d.). Otto Bock quality for life. Retrieved April 14, 2013, from C-Leg Product Line:

http://www.ottobock.com/cps/rde/xbcr/ob_cn_zh/im_646a231_gb_product_line_c -leg_prothetics.pdf

Popusoi, A. (2009, April 22). Next generation of the Power Knee from Ossur. Retrieved April 14, 2013, from http://gadgets.softpedia.com/news/Next-generation-of-thePower-Knee-from-Ossur-2499-01.html

Reid supply company. (n.d.). Retrieved May 15, 2013, from http://www.reidsupply.com/sku/SHB-13/

Robots, A. O. (n.d.). Bionic knee. Retrieved April 14, 2013, from http://www.allonrobots.com/bionic-knee.html

Tan, M. (2012, March 14). DoD says amputations reached wartime high. Retrieved April 11,2013 , from http://www.armytimes.com/article/20120314/NEWS/203140318/DoD-saysamputations-reached-wartime-high

The new functions. (2011). Retrieved April 13, 2013, from http://cleg.ottobock.com/en/funktionen.php

The technology behind the success of the Rheo Knee. (n.d.). Retrieved April 13, 2013, from http://www.ossur.com/?PageID=15766

The technology of the Power Knee. (n.d.). Retrieved April 13, 2013, from https://www.ossur.com/?PageID=15767

Thomasnet. (n.d.). Retrieved May 14, 2013, from http://news.thomasnet.com/companystory/Tapco-Adds-Western-3-ProngElevator-Bolt-in-Stainless-Steel-565552

Trigger point performance therapy: Quads \& IT bands. (n.d.). Retrieved April 15, 2013, from http://www.tptherapy.com/unlock-your-body-anatomy-quads.html

Vallabhajosula, S., Yentes, J. M., \& Stergiou, N. (2012). Frontal joint dynamics when initiating stair ascent from a walk versus a stand. Journal of Biomechanics, Volume 45: 609-613.

webpage, C. n. (2006, July 8). High-tech prosthetics. Retrieved April 14, 2013, from http://news.cnet.com/2300-11393_3-6091199-1.html 
Wenke, J. C., Krueger, C. A., \& Ficke, J. R. (2012). Ten years at war: Comprehensive analysis of amputation trends. Journal of Trauma and Acute Care Surgery.

Zahedi, S. (n.d.). Lower limb prosthetic research in the 21 st century. 
APPENDIX A: Technical Drawings
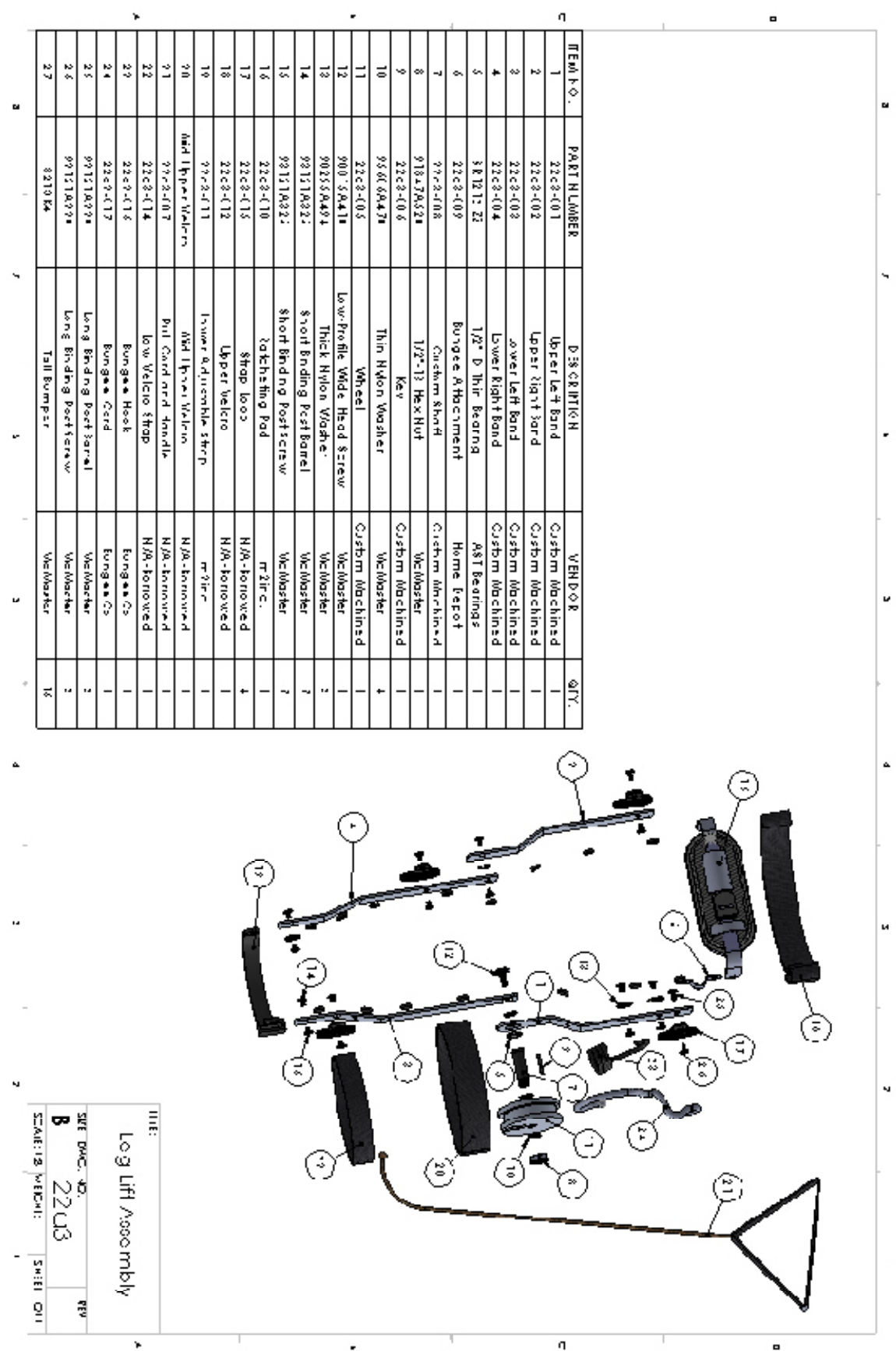


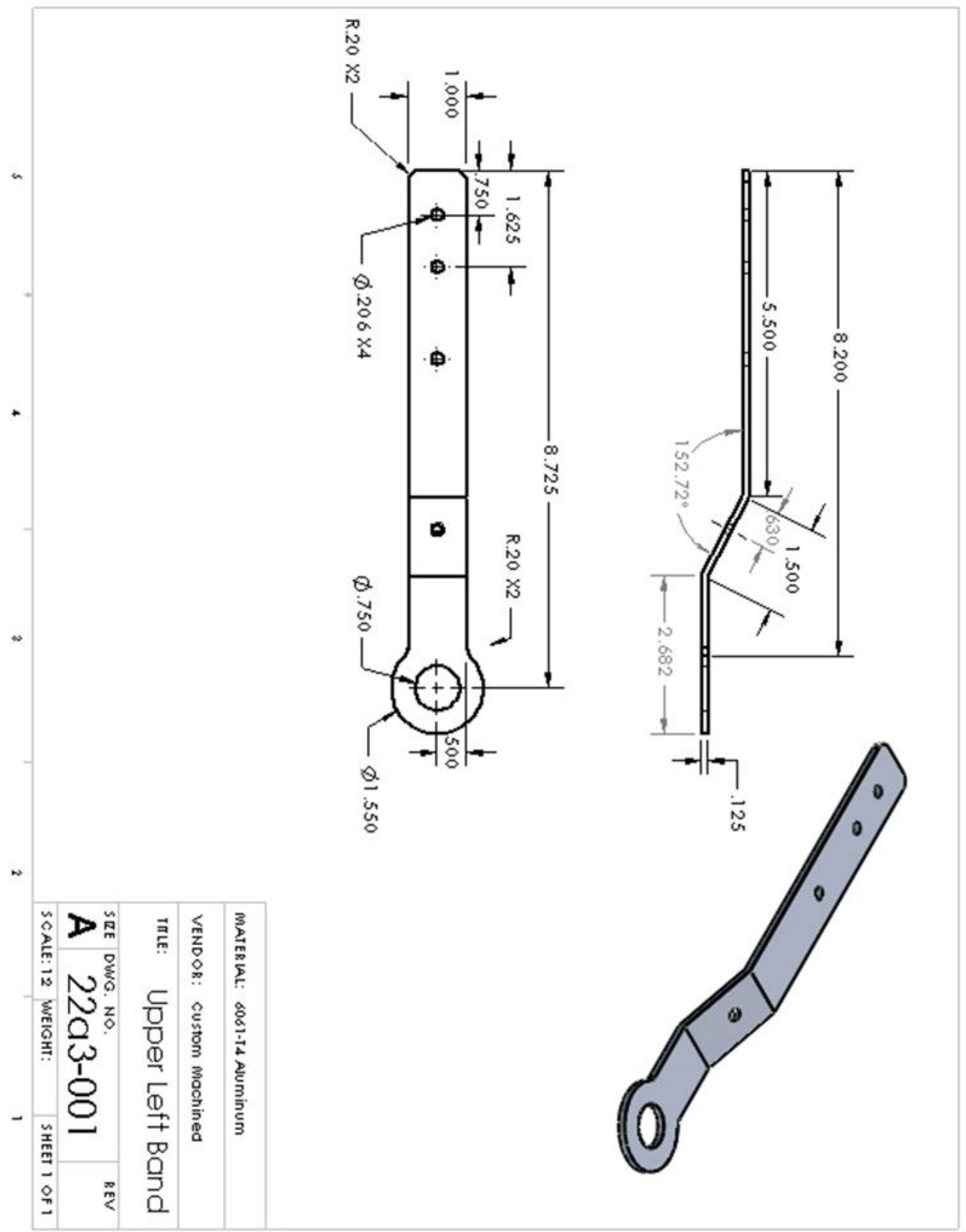




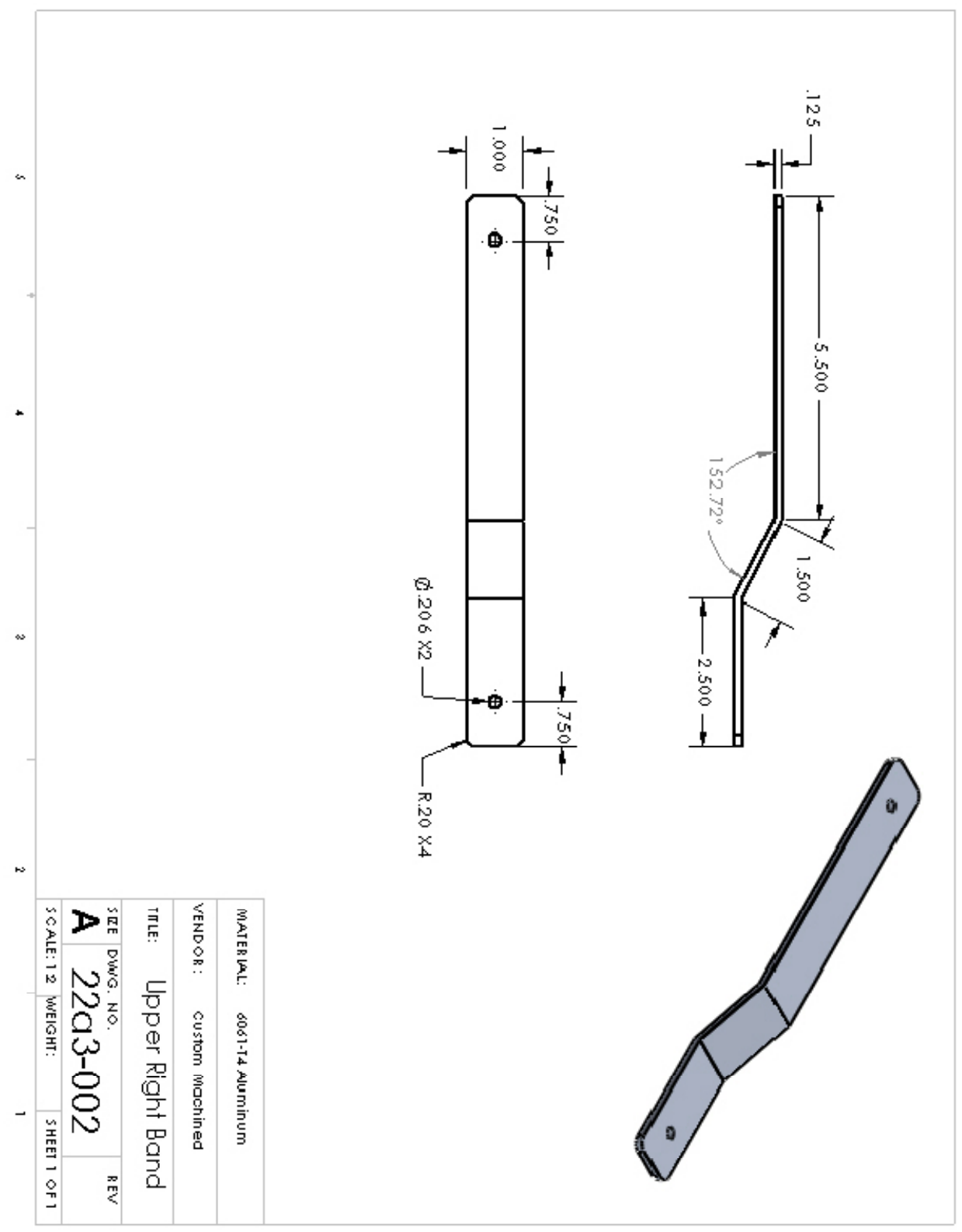




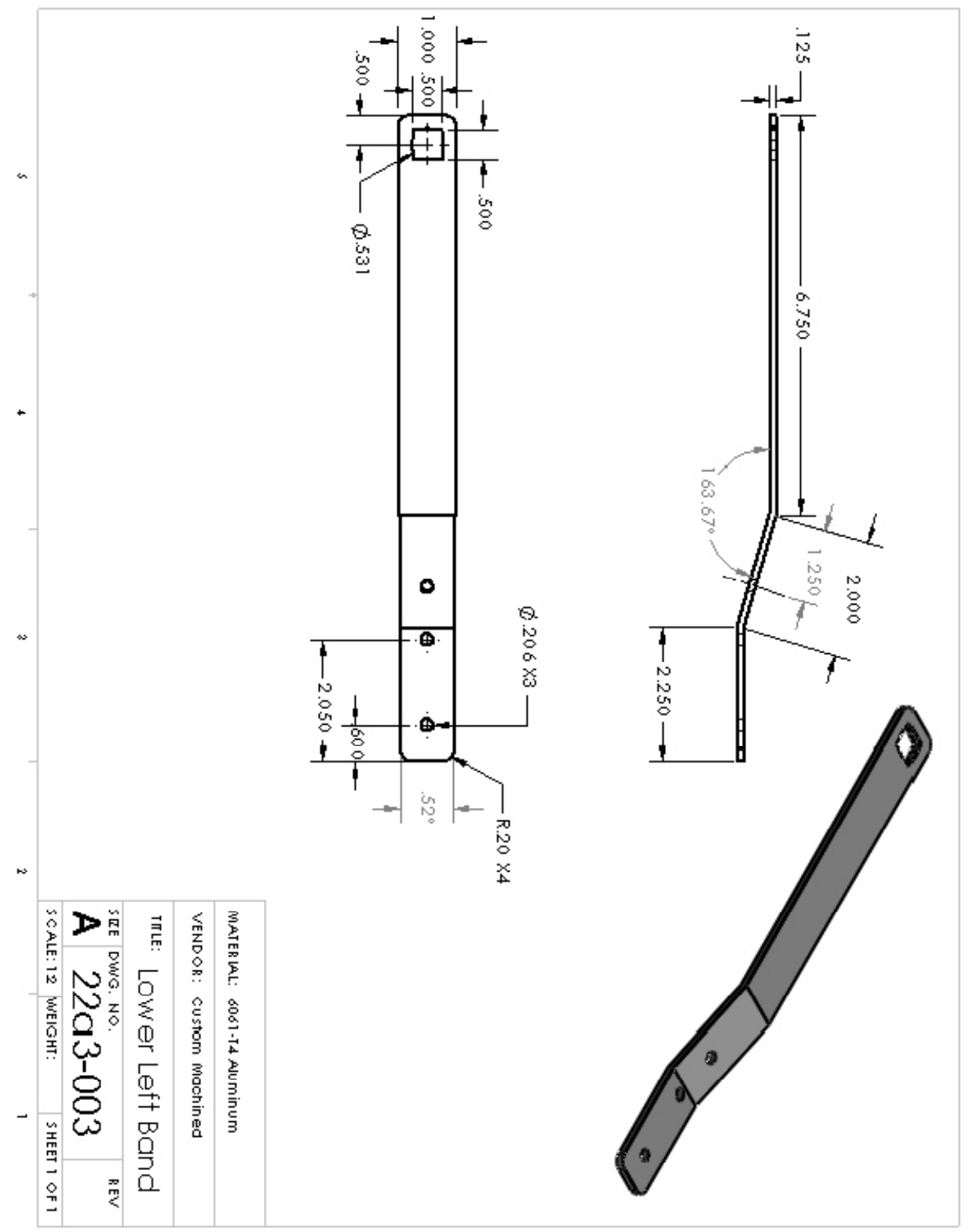




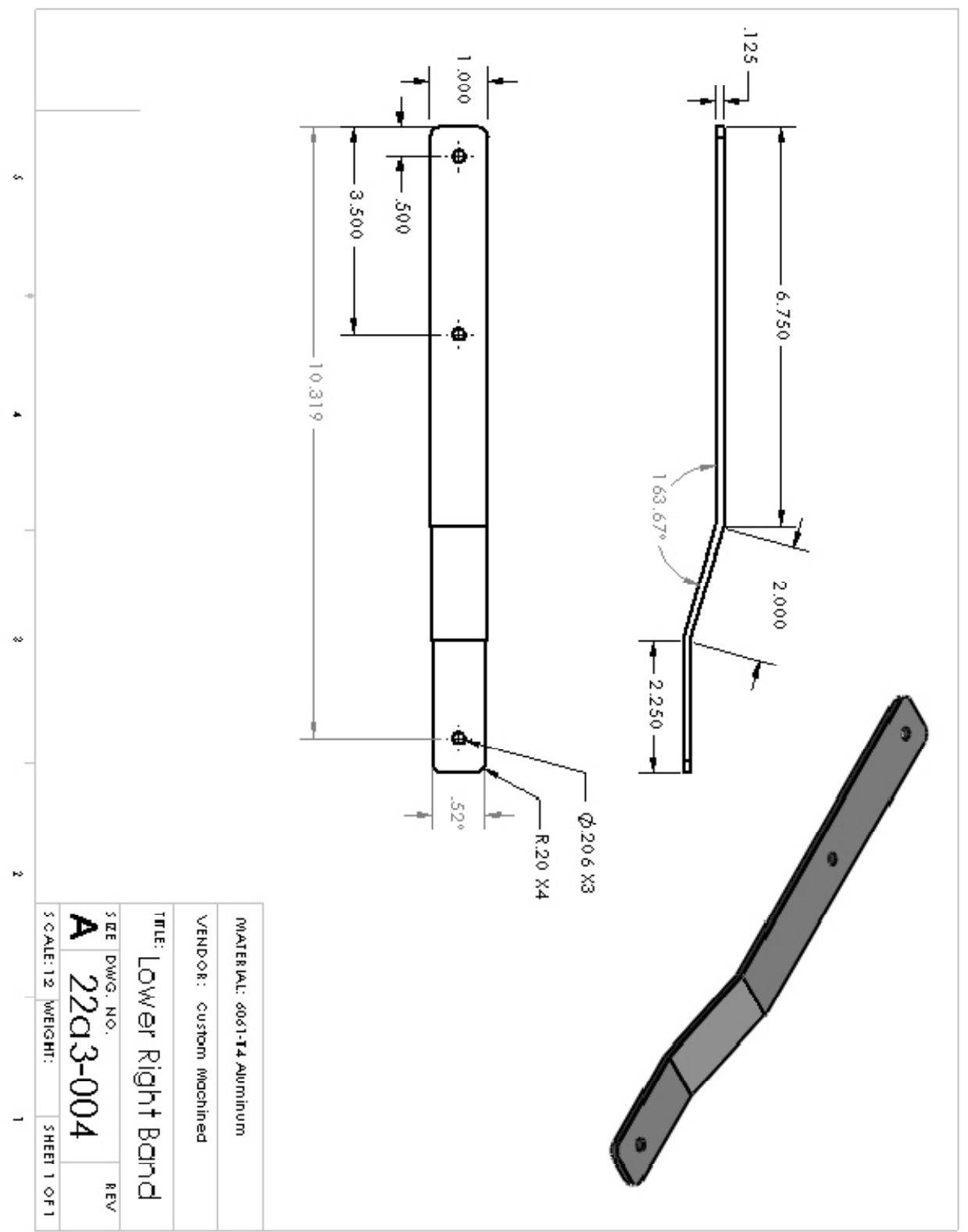




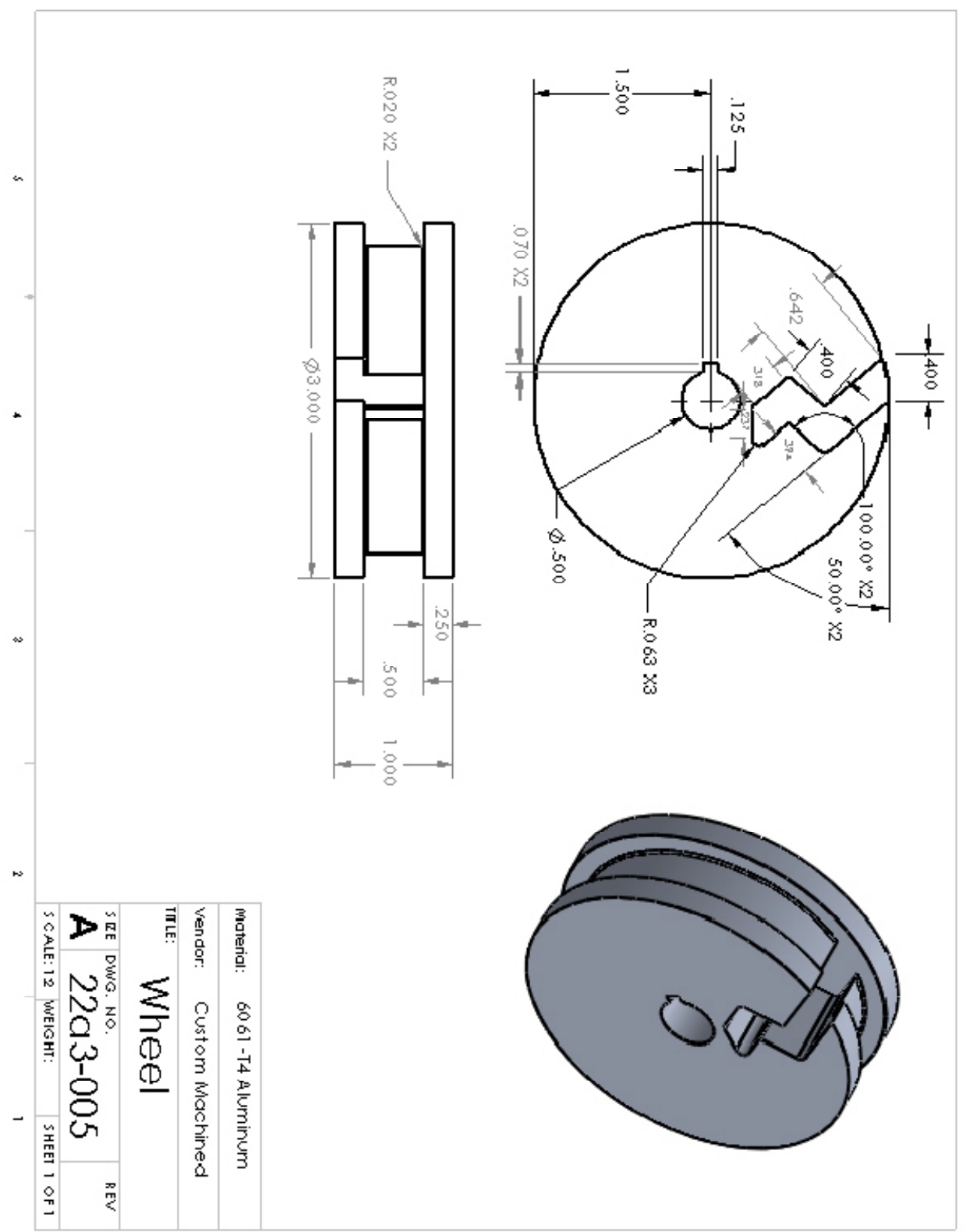




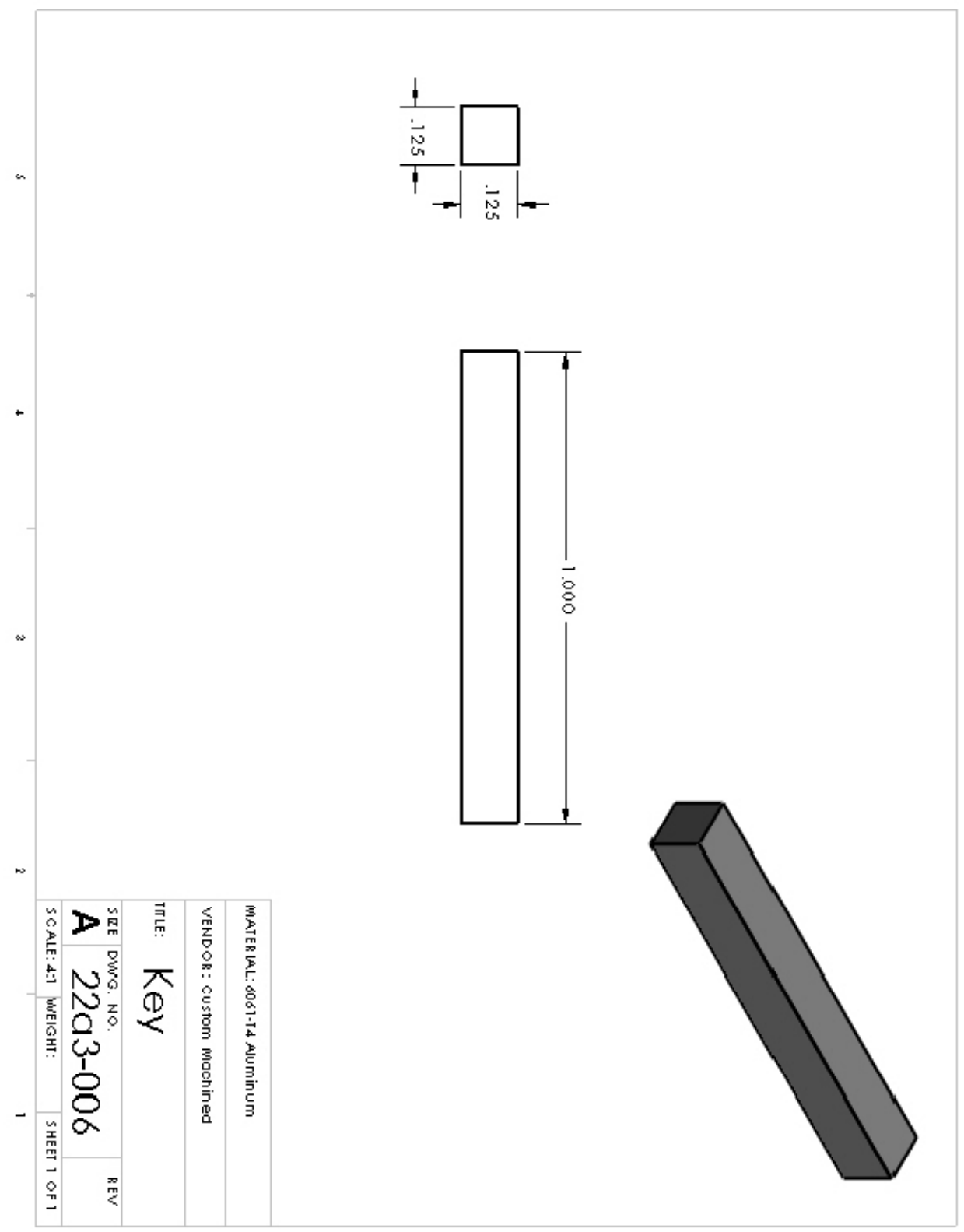




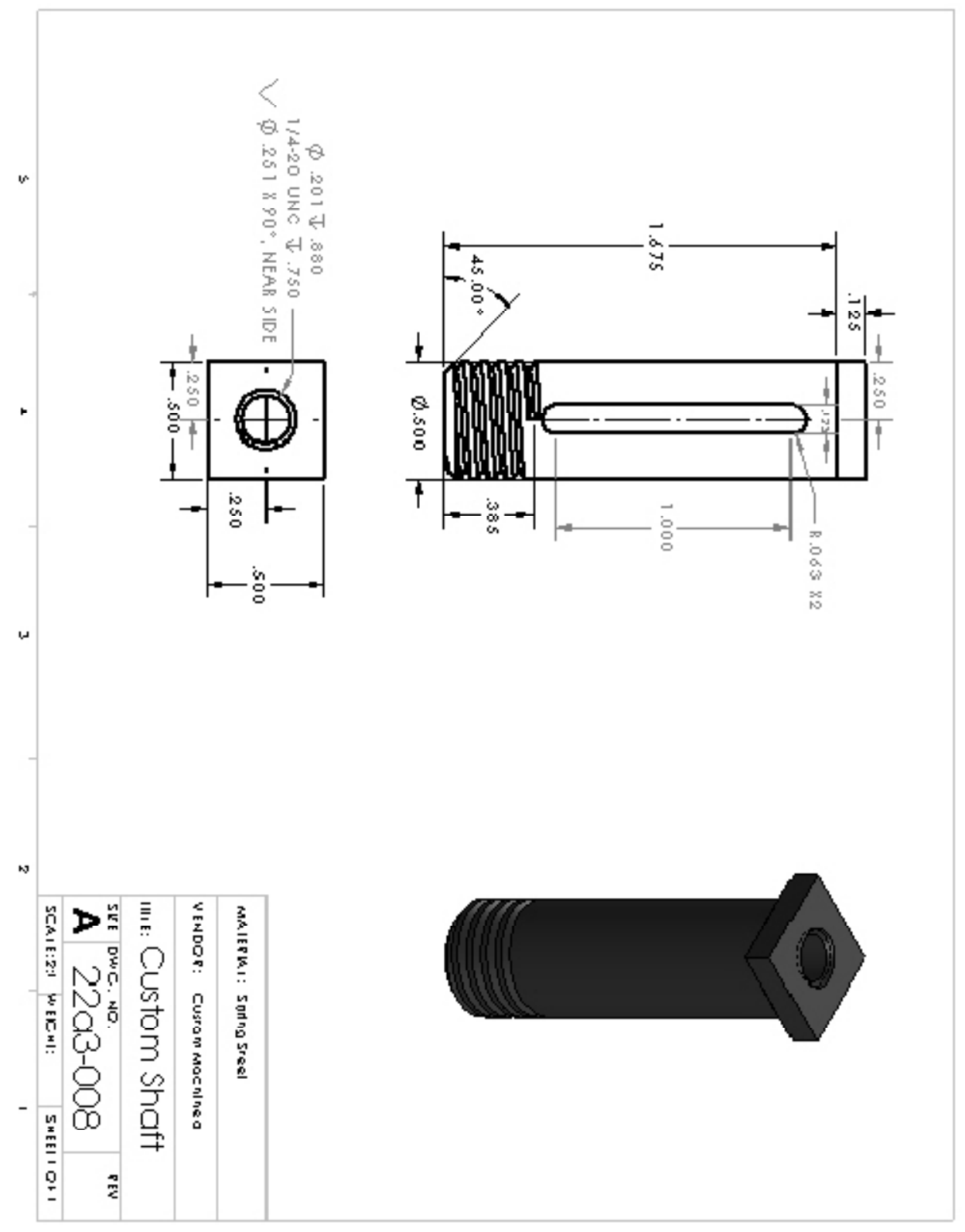


APPENDIX B: Testing Evaluation Forms

\section{Evaluation of Stair Assist Device for AK Amputees}

\begin{tabular}{|c|c|}
\hline Date: & 29 Apnl 2013 \\
\hline Sex: & MALE / FEMALE (circle one) \\
\hline Height: & $5^{\prime} 10^{\prime \prime}$ \\
\hline Weight: & 175 \\
\hline $\begin{array}{l}\text { Knee Make \& } \\
\text { Model: }\end{array}$ & Total Knee Ossur \\
\hline
\end{tabular}

Please rate the following criteria on a scale from 1-10:

$1=$ Not at all satisfied

$10=$ Completely satisfied

Before Stair Climbing

Weight of Device:

Aesthetics:

Size and Bulkiness:

Comfort:

Adjustability:

Ergonomics (ease of setup and fitting):

$\begin{array}{llllllllll}1 & 2 & 3 & 4 & 5 & 6 & 7 & 8 & 9 & 10 \\ 1 & 2 & 3 & 4 & 5 & 6 & 7 & 8 & 9 & 10 \\ 1 & 2 & 3 & 4 & 5 & 6 & 7 & 8 & 9 & 10 \\ 1 & 2 & 3 & 4 & 5 & 6 & 7 & 8 & 9 & 10 \\ 1 & 2 & 3 & 4 & 5 & 6 & 7 & 8 & 9 & 10 \\ 1 & 2 & 3 & 4 & 5 & 6 & 7 & 8 & 9 & 10\end{array}$

During Stair Climbing

Force Generated:

Effective Assist:

Balance of Patient:

Pull Handle Force:

\begin{tabular}{llllllllll}
\multicolumn{10}{c}{ Circle One Number } \\
1 & 2 & 3 & 4 & 5 & 6 & 7 & 8 & 9 & 10 \\
1 & 2 & 3 & 4 & 5 & 6 & 7 & 8 & 9 & 10 \\
1 & 2 & 3 & 4 & 5 & 6 & 7 & 8 & 9 & 10 \\
1 & 2 & 3 & 4 & 5 & 6 & 7 & 8 & 9 & 10
\end{tabular}

**Was a handrail used for balance and stability during testing? YES)/ No (circle one) Along with Z staff
assist for saloty

COMMENTS: Casery the overall cllect was honestly manginal on th total line. The assist ePloA actually was a hinderence en tho stairs in general onl afledel overall balonea. The one positire point with the fotal knec was that it did assist dering normal - Carl ambulation but horesthy wot to a point that the patient could went to wear the clevize on a requelor basis. 


\section{Evaluation of Stair Assist Device for AK Amputees}

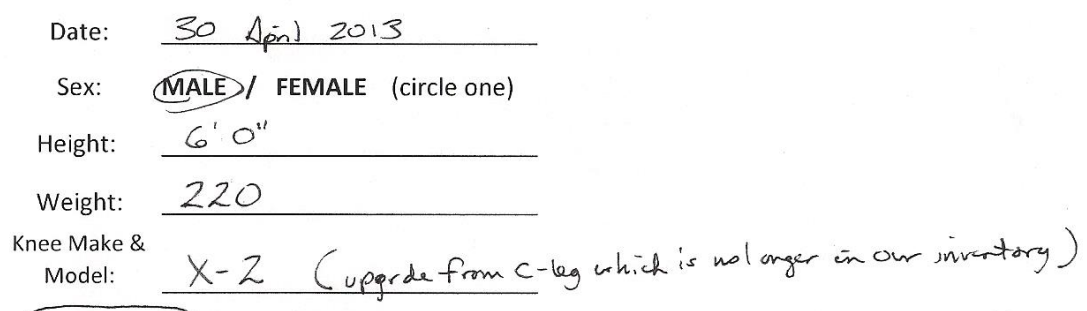

Unilateral / Bilateral (circle one)

Please rate the following criteria on a scale from 1-10:

$$
\begin{gathered}
1=\text { Not at all satisfied } \\
10=\text { Completely satisfied }
\end{gathered}
$$

Circle One Number

Before Stair Climbing

Weight of Device:

Aesthetics:

Size and Bulkiness:

Comfort:

Adjustability:

Ergonomics (ease of setup and fitting):

$\begin{array}{cccccccccc}1 & 2 & 3 & 4 & 5 & 6 & 7 & 8 & 9 & 10 \\ 1 & 2 & 3 & 4 & 5 & 6 & 7 & 8 & 9 & 10 \\ 1 & 2 & 3 & 4 & 5 & 6 & 7 & 8 & 9 & 10 \\ 1 & 2 & 3 & 4 & 5 & 6 & 7 & 8 & 9 & 10 \\ 1 & 2 & 3 & 4 & 5 & 6 & 7 & 8 & 9 & 10 \\ 1 & 2 & 3 & 4 & 5 & 6 & 7 & 8 & 9 & 10\end{array}$

During Stair Climbing

Force Generated:

Effective Assist:

Balance of Patient:

Pull Handle Force:

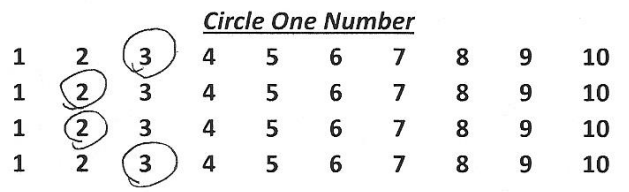

**Was a handrail used for balance and stability during testing? (YES) NO (circle one) +2 stafl on stand by

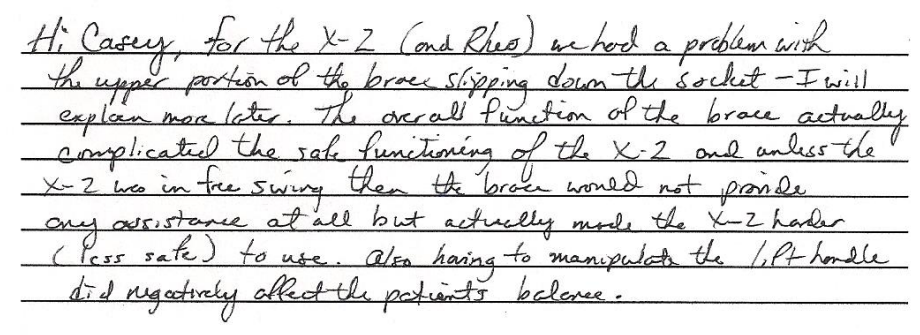




\section{Evaluation of Stair Assist Device for AK Amputees}

\begin{tabular}{|c|c|}
\hline Date: & 30 April 2013 \\
\hline Sex: & MALE FEMALE (circle one) \\
\hline Height: & $6^{\prime} 2^{\prime \prime}$ \\
\hline Weight: & 185 \\
\hline $\begin{array}{l}\text { Knee Make \& } \\
\text { Model: }\end{array}$ & Rheo \\
\hline
\end{tabular}

Please rate the following criteria on a scale from 1-10:

$\mathbf{1}=$ Not at all satisfied $10=$ Completely satisfied

Circle One Number

Before Stair Climbing

Weight of Device:

Aesthetics:

Size and Bulkiness:

Comfort:

Adjustability:

Ergonomics (ease of setup and fitting):

$\begin{array}{llllllllll}1 & 2 & 3 & 4 & 5 & 6 & 7 & 8 & 9 & 10 \\ 1 & 2 & 3 & 4 & 5 & 6 & 7 & 8 & 9 & 10 \\ 1 & 2 & 3 & 4 & 5 & 6 & 7 & 8 & 9 & 10 \\ 1 & 2 & 3 & 4 & 5 & 6 & 7 & 8 & 9 & 10 \\ 1 & 2 & 3 & 4 & 5 & 6 & 7 & 8 & 9 & 10 \\ 1 & 2 & 3 & 4 & 5 & 6 & 7 & 8 & 9 & 10\end{array}$

During Stair Climbing

Force Generated:

Effective Assist:

Balance of Patient:

Pull Handle Force:

\begin{tabular}{cccccccccc}
\multicolumn{11}{c}{ Circle One Number } \\
1 & 2 & 3 & 4 & 5 & 6 & 7 & 8 & 9 & 10 \\
1 & 2 & 3 & 4 & 5 & 6 & 7 & 8 & 9 & 10 \\
1 & 2 & 3 & 4 & 5 & 6 & 7 & 8 & 9 & 10 \\
1 & 2 & 3 & 4 & 5 & 6 & 7 & 8 & 9 & 10
\end{tabular}
**Was a handrail used for balance and stability during testing? YES / NO (circle one) +2 staff assist for
safely.

COMMENTS:

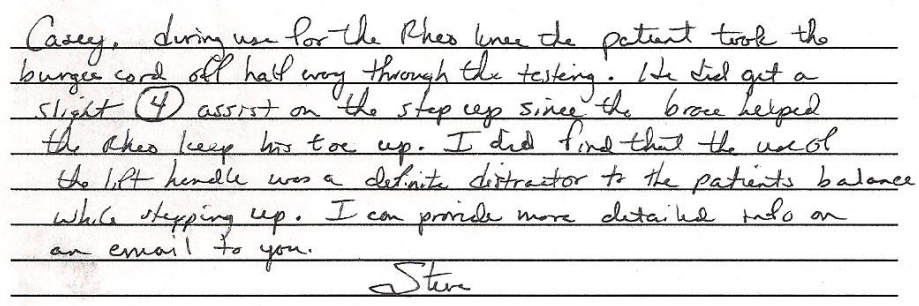

